\title{
ADAPTIVE ECHO CANCELLATION ANALYSIS
}

\author{
by \\ Yongdong Sun \\ (B.Eng., Shenyang, China, June 1993)
}

\begin{abstract}
A Project
presented to Ryerson University

in partial fulfillment of the

requirements for the degree of
\end{abstract}

Master of Engineering

in the Program of

Electrical and Computer Engineering

Toronto, Ontario, Canada, 2004

(C) Yongdong Sun 2004 


\section{UMI Number: EC52987}

\section{All rights reserved \\ INFORMATION TO USERS}

The quality of this reproduction is dependent upon the quality of the copy submitted. Broken or indistinct print, colored or poor quality illustrations and photographs, print bleed-through, substandard margins, and improper alignment can adversely affect reproduction.

In the unlikely event that the author did not send a complete manuscript and there are missing pages, these will be noted. Also, if unauthorized copyright material had to be removed, a note will indicate the deletion.

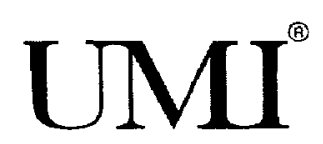

UMI Microform EC52987

Copyright 2008 by ProQuest LLC

All rights reserved. This microform edition is protected against unauthorized copying under Title 17, United States Code.

ProQuest LLC

789 East Eisenhower Parkway

P.O. Box 1346

Ann Arbor, MI 48106-1346 


\section{Author's Declaration}

I hereby declare that I am the sole author of this Research Paper.

I authorize Ryerson University to lend this Research Paper to other institutions or individuals for the purpose of scholarly research.

Signature

I further authorize Ryerson University to reproduce this Research Paper by photocopying or by other means, in total or in part, at the request of other institutions or individuals for the purpose of scholarly research.

Signature 


\section{Borrow List}

Ryerson University requires the signatures of all persons using or photocopying this thesis. Please sign below, and give address and date. 


\begin{abstract}
Title: Adaptive Echo Cancellation Analysis

Echo cancellation is a classic problem in DSP and digital communication. Adaptive echo cancellation is an application of adaptive filtering to the attenuation of undesired echo in the telecommunication network. This is accomplished by modeling the echo path using an adaptive filter and subtracting the estimated echo from the echo-path output. In this project, the concept of echo cancellation and echo cancellation systems are studied, simulated, and implemented in Matlab and TI TMS320C6711 DSK. The LMS, NLMS, Fast Block LMS and RLS algorithm are investigated for echo canceller and two double talk detection algorithms: the Geigel algorithm and the normalized crosscorrelation algorithm are presented and combined with NLMS adaptive algorithm against double talk. Finally The adaptive echo cancellation system successfully developed by the NLMS and normalized cross-correlation DTD algorithms meet the general ITU G. 168 requirements and show excellent robustness against double talk.
\end{abstract}




\section{Acknowledgements}

Many thanks to Dr. Mike Kassam, my supervisor, for his thoughtful advice, assistance and comments.

Also, thanks to my wife and my family for supporting me during this project. 


\section{Table of Contents}

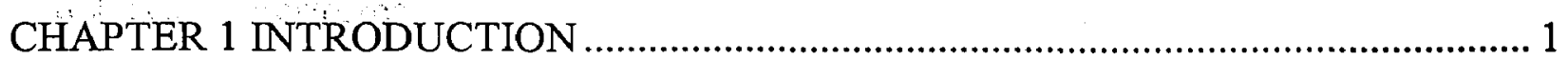

1.1 Adaptive Echo Cancellation .............................................................................. 1

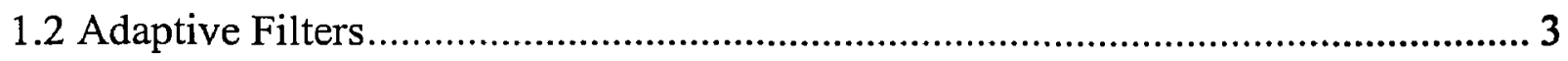

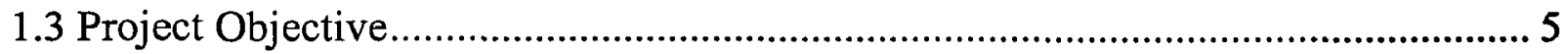

CHAPTER 2 ECHO CANCELLATION ADAPTIVE METHODS .................................. 6

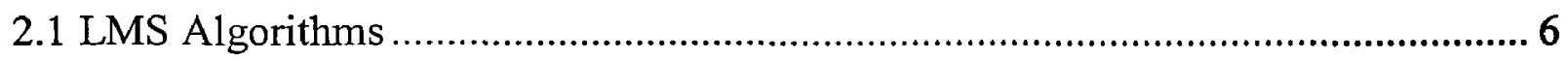

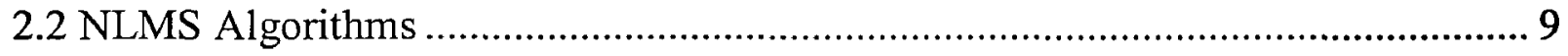

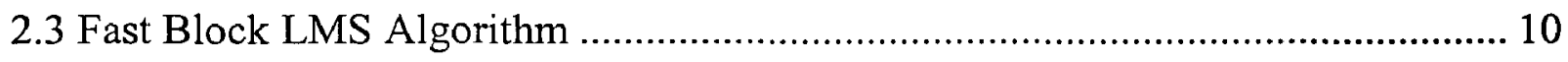

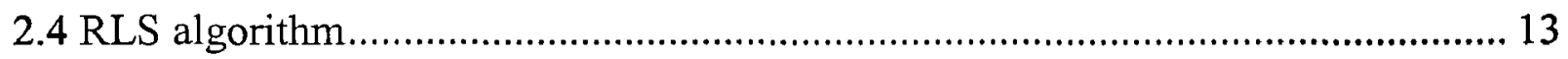

CHAPTER 3 DOUBLE TALK DETECTION ALGORITHMS ................................... 15

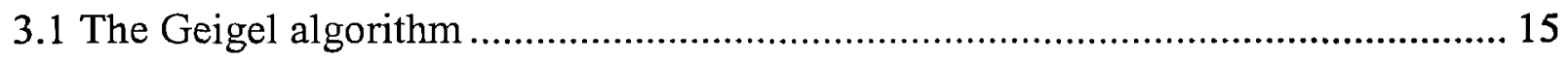

3.2 Normalized cross-correlation algorithm ........................................................... 16

3.3 Evaluation procedure of double detectors........................................................ 18

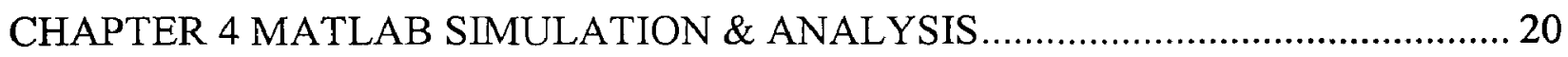

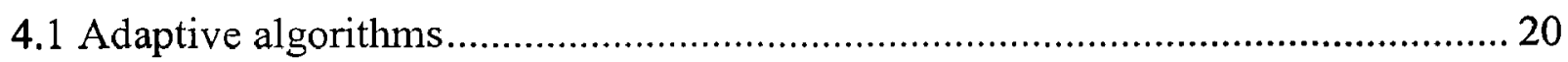

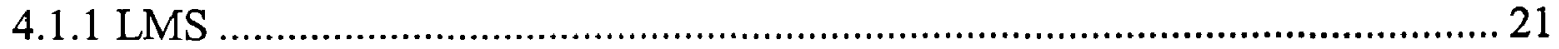

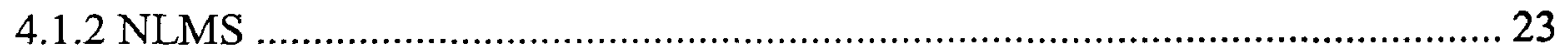

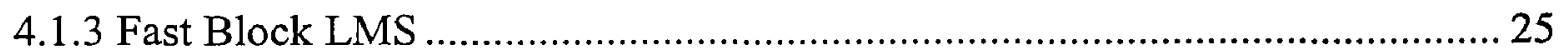

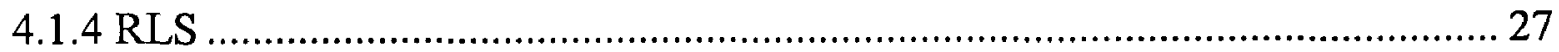

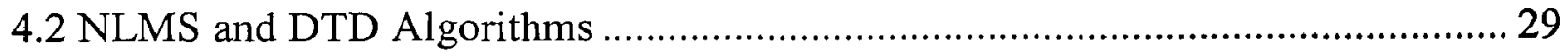

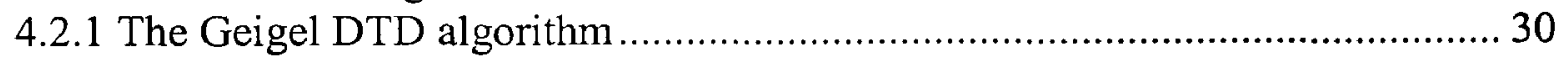

4.2.2 NLMS and normalized cross-correlation DTD algorithm ............................. 33

4.3 Matlab Simulation Summary …….................................................................... 35

CHAPTER 5 ADAPTIVE ECHO CANCELLER WITH DTD STRUCTURE............... 39

5.1 Adaptive Echo Canceller with DTD ..................................................................... 39

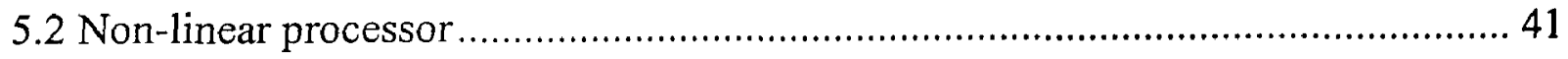

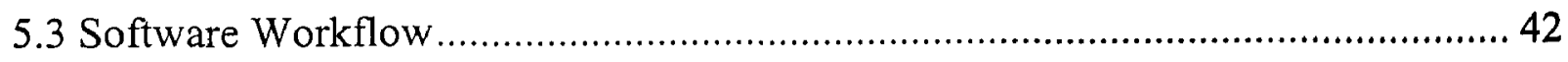

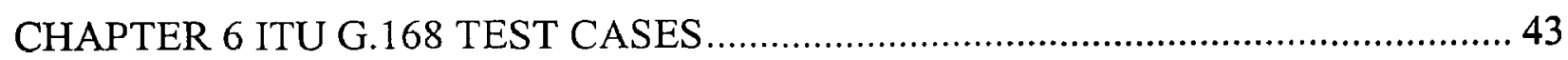

6.1 Test 1: Steady state residual and residual echo level test ........................................43

6.2 Test 2A and 2B: Convergence test with NLP enabled and disabled ...................... 44

6.2.1 Test 2A: Convergence test with NLP enabled.................................................. 44

6.2.1 Test 2B: Convergence test with NLP disabled .............................................. 44 
6.3 Test 2C: Convergence test in the presence of background noise............................ 45

6.4 Test 3: Performance under conditions of double talk ............................................ 47

6.4.1 Test 3A: Double talk test with low near end levels ...................................... 47

6.4.2 Test 3B: Double talk test with high near end levels ....................................... 48

6.5 Test 3C: Double talk test under simulated conversation .................................... 50

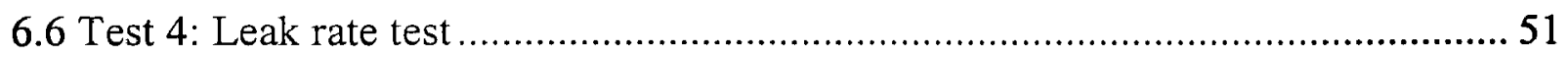

6.7 Test 5: Infinite return loss convergence test ...................................................... 52

6.8 Test 6: Non-divergence on narrow-band signals ...............................................5 53

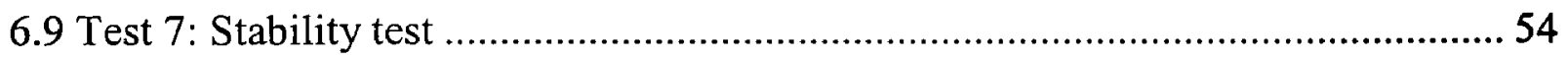

6.10 Test 8: Non-convergence, in-band signaling, and continuity check tones ............ 55

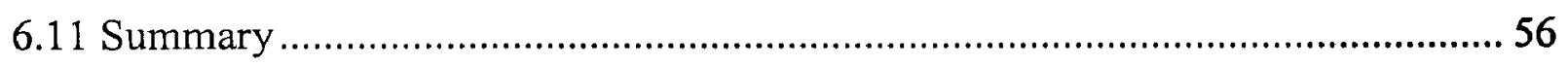

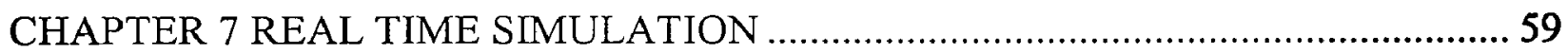

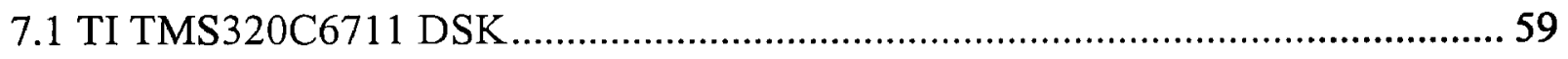

7.2 C6711 DSK Simulation using Code Composer Studio .......................................... 59

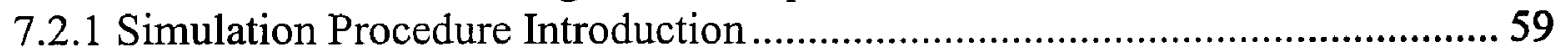

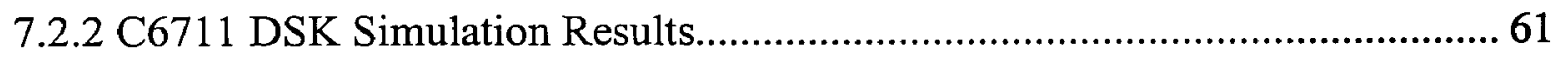

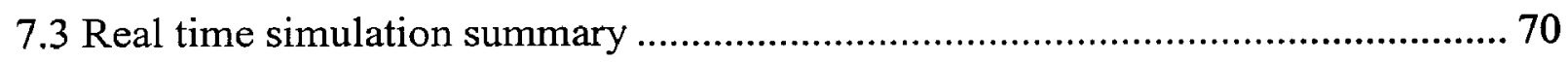

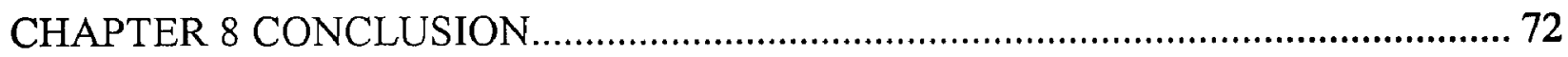

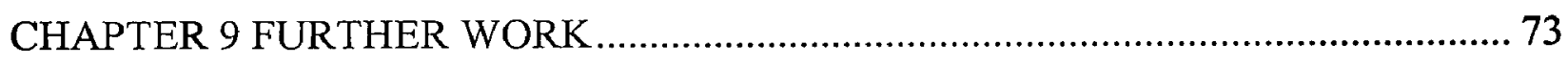

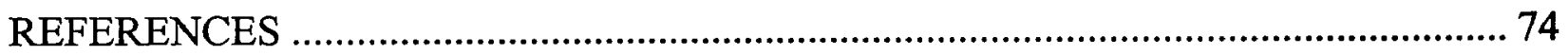

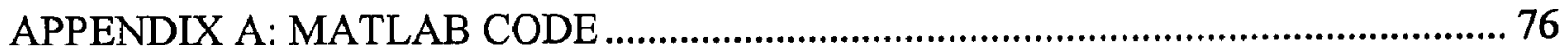

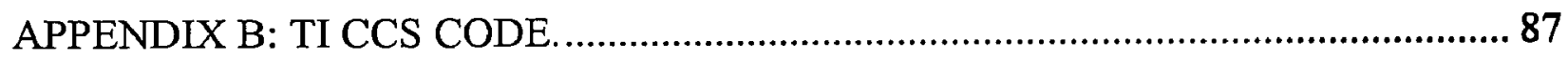




\section{Chapter 1}

\section{Introduction}

\subsection{Adaptive Echo Cancellation}

Echo is a phenomenon in which a delayed and distorted version of an original sound or electrical signal is reflected back to the source. In real life, echoes often occurrence among conversations. The echoes of speech waves can be heard as they are reflected from the floor, wall and other neighboring objects. In such a case when the reflected wave arrives a few tens of milliseconds delay after the direct sound, it can be heard as an obvious echo. These echoes are bothering and may unexpectedly interrupt a conversation. Thus it is desired to eliminate these echoes. In telephone communication, there are two main types of echo: network and acoustic echoes. The network echo results from the impedance mismatch at points along the transmission line, for example, at the hybrids of a public switched telephony network (PSTN) exchange, where the subscriber two-wire lines are connected to four-wire lines. Acoustic echo is due to the acoustic coupling between the loudspeaker and microphone in hands-free telephone systems, for example, if a communication is between one or more hands-free telephones (or speaker phones), then acoustic feedback paths are set up between the telephone's loudspeaker and microphone at each end. Acoustic Echo is more hostile than network echo mainly because of the different nature of echo paths.

The solution to these echo problems is to eliminate the echo with an echo suppressor or echo canceller. 
The problems with echo suppressor are:

- only one speaker can talk at a time

- clips speech

- take time to detect the beginning of speech

Now using adaptive filter to reduce the echo and increase communication quality is a common technology in communication system, but there are still some challenges :

- many existing adaptive algorithms give different performance

- different adaptive algorithms need different parameter setting

- overcome double talk disturbance

This project focuses on the following achievements:

(1) Providing an easy approach to compare and review various adaptive algorithms based on their convergence rate, steady state ERLE and complexity of implementation, etc.

(2) Supplying different pre-designed double talk detection methods that can be easily configured with different parameter to obtain the expected performance.

(3) Implementation of AEC and DTD algorithms to work practically and efficiently using smooth estimation and the pre-set parameters selected from experiments.

Adaptive cancellation of such acoustic echo has became very important in hands-free communication systems, e.g. tele-conference, video-conference and $\mathrm{PC}$ telephony systems. 
Echo canceller is a better solution to the acoustic echo problem, which allows both speakers to talk at the same time.

The Basic Structure of Adaptive Echo Canceller is shown in Figure 1.1 and the estimated echo signal $y(t)$ generated by an adaptive filter eliminates the echo signal $y(t)$. The coefficients of the adaptive filter are adjusted by adaptive algorithm according to the estimation error signal $e(t)$.

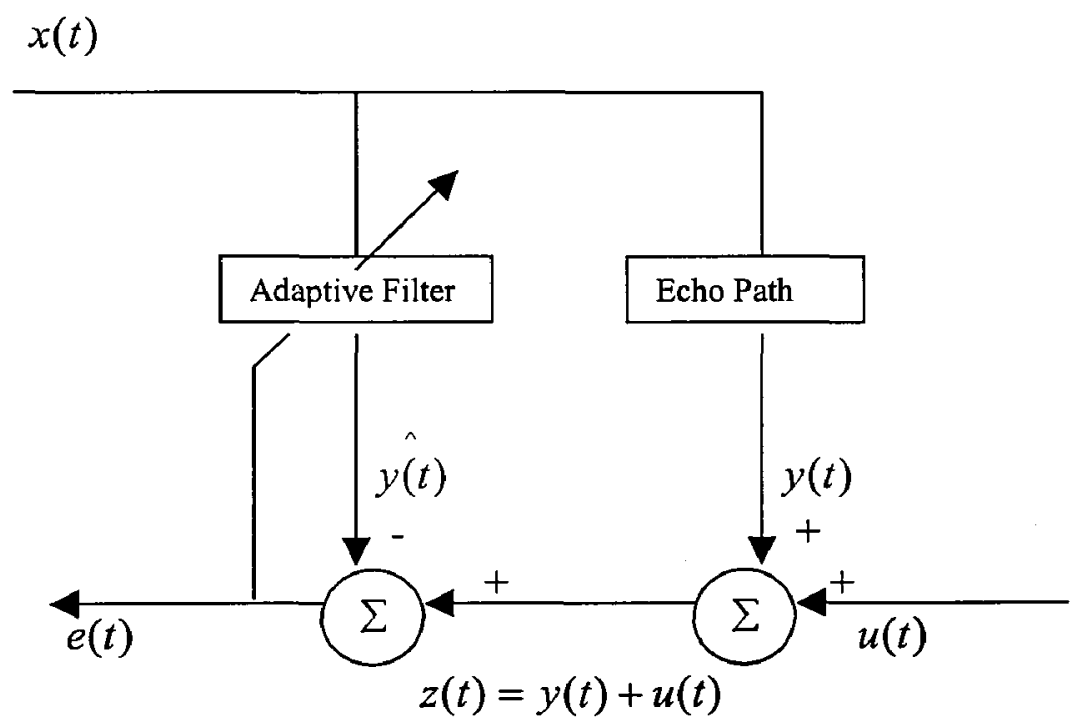

Figure 1.1 Basic structure of adaptive echo canceller: far end signal $x(t)$, echo signal $y(t)$, near end signal $u(t)$, received signal $z(t)$, estimated echo signal $\hat{y(t)}$ and error signal $e(t)$.

\subsection{Adaptive Filters}

Adaptive usually deploys a traversal Finite Impulse Response (FIR) structure due to its guaranteed stability and the adaptation of the FIR filter coefficients is controlled by an adaptive algorithm. The adaptive algorithm is the heart of an AEC, which decides the convergence behavior and tracking behavior of the AEC. The tracking behavior indicates 
how fast the adaptive filter can follow enclosure dislocations, whereas the convergence behavior is studied as an initial adjustment of the adaptive filter to the impulse response of the room or car.

In Figure 1.2, w represents the coefficients of the FIR filter tap weight vector, $\mathbf{x}(\mathbf{k})$ is the input vector samples, $z^{-1}$ is the delay unit, $\mathrm{y}(\mathrm{k})$ is the adaptive filter output, $d_{k}$ is the desired echoed signal and $\varepsilon_{k}$ is the estimation error at time $\mathrm{k}$.

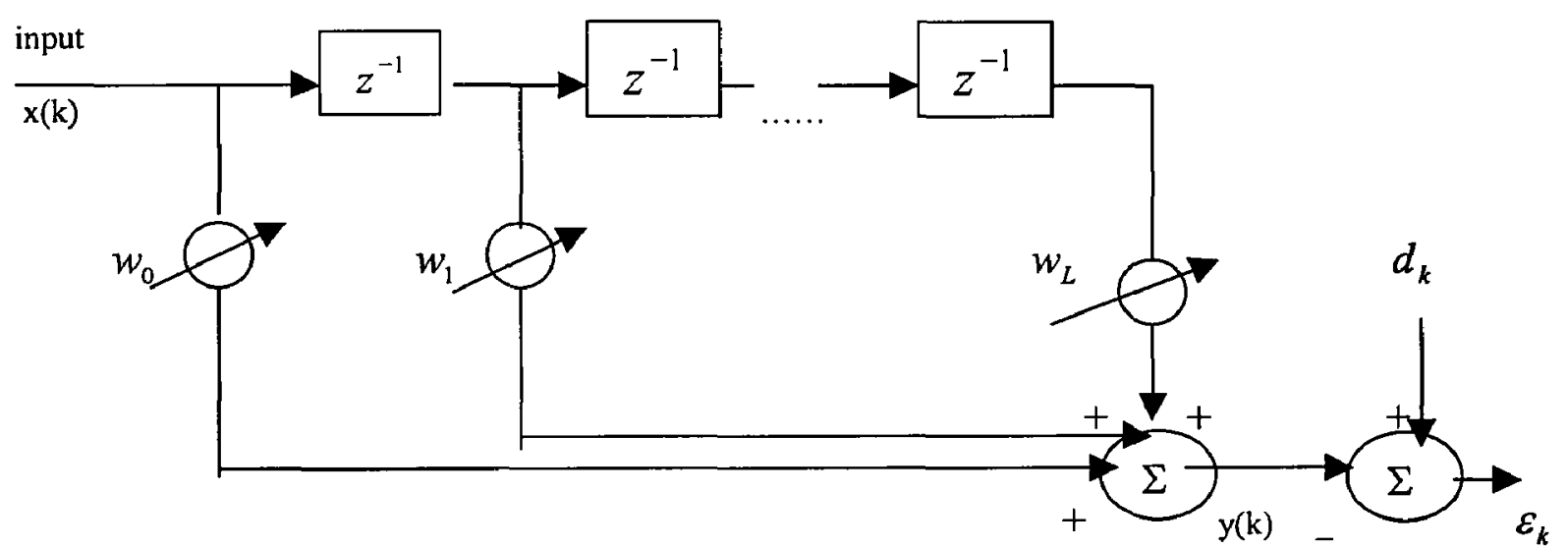

Figure 1.2 Adaptive filter block diagram

The adaptive filter is used to calculate the difference between the desired signal and the adaptive filter output, $\varepsilon_{k}$. This error signal is fed back into the adaptive filter and its coefficients are changed algorithmically to minimize the cost function that is a function of $\varepsilon_{k}$. In acoustic echo cancellation, the optimal output of the adaptive filter is the value of the unwanted echoed signal. When the adaptive filter output is equal to desired signal, the error signal becomes 0 , in this ideal situation, the echo signal will be completely cancelled and the far end user will not hear his original speech returned to him. 


\subsection{Project Objective}

In the project, the LMS, NLMS, Fast Block LMS and RLS algorithm are studied and alternatively used in adaptive acoustic echo canceller. The NLMS with two different Double Talk detection algorithms are integrated in the adaptive echo canceller against double talk. The performance and parameters of these adaptive algorithms such as filter length, step size and convergence speed are studied in details and the two Double Talk algorithms: detection algorithms: the Geigel algorithm and the normalized crosscorrelation algorithm are studied and simulated with NLMS algorithm in Matlab and TI TMS320C6711 DSK. The ITU G.168 standard is used to study the performance of the adaptive echo canceller. 


\section{Chapter 2}

\section{Echo Cancellation Adaptive Methods}

Most echo cancellers use variants of the LMS adaptation algorithm [1] [2][3]. The attractions of the LMS are its relatively low memory and computational requirements and its ease of implementation and monitoring. In practice, LMS adaptation has produced effective line echo cancellation systems.

\subsection{LMS Algorithms}

In Figure 1.1, the error signal $\varepsilon_{k}$ is

$$
\varepsilon_{k}=\mathrm{d}_{k}-\mathrm{y}_{k}
$$

and

$$
\mathrm{y}_{k}=\mathrm{X}_{k}^{T} \mathrm{~W}=\mathrm{W}^{T} \mathrm{X}_{k}
$$

So,

$$
\varepsilon_{k}=\mathrm{d}_{k}-\mathrm{X}_{k}^{T} \mathrm{~W}=\mathrm{d}_{k}-\mathrm{W}^{T} \mathrm{X}_{k}
$$

We can square $\varepsilon_{k}$ to get the instantaneous squared error,

$$
\begin{aligned}
& \varepsilon_{k}^{2}=\mathrm{d}_{k}^{2}+\mathrm{W}^{T} \mathrm{X}_{k} \mathrm{X}_{k}^{T} \mathrm{~W}-2 \mathrm{~d}_{k} \mathrm{X}_{k}^{T} \mathrm{~W} \\
& \mathrm{E}\left[\varepsilon_{k}^{2}\right]=\mathrm{E}\left[\mathrm{d}_{k}^{2}\right]+\mathrm{W}^{T} \mathrm{E}\left[\mathrm{X}_{k} \mathrm{X}_{k}^{T}\right] \mathrm{W}-2 \mathrm{E}\left[\mathrm{d}_{k} \mathrm{X}_{k}^{T}\right] \mathrm{W}
\end{aligned}
$$

Let $\mathrm{R}$ be defined as the autocorrelation matrix

$$
R=E\left[X_{k} X_{k}^{T}\right]=E\left\{\begin{array}{cccc}
x_{k} x_{k} & x_{k} x_{k-1} & \cdots & x_{k} x_{k-N+1} \\
x_{k-1} x_{k} & x_{k-1} x_{k-1} & \cdots & x_{k-1} x_{k-N+1} \\
\vdots & \vdots & & \vdots \\
x_{k-N+1} x_{k} & x_{k-N+1} x_{k-1} & \cdots & x_{k-N+1} x_{k-N+1}
\end{array}\right\}
$$


Let $\mathrm{P}$ be similarly defined as the cross-correlation matrix

$$
\mathrm{P}=E\left[\mathrm{~d}_{k} \mathrm{X}_{k}^{J}\right]=E\left\{\begin{array}{c}
d_{k} x_{k} \\
d_{k} x_{k-1} \\
\vdots \\
d_{k} x_{k-N+1}
\end{array}\right\}
$$

And the mean-square error can be designated by $\xi$ and we can obtain the following expression:

$$
\mathrm{MSE} \cong \xi=\mathrm{E}\left[\varepsilon_{k}^{2}\right]=\mathrm{E}\left[\mathrm{d}_{k}^{2}\right]+\mathrm{W}^{T} \mathrm{RW}-2 \mathrm{PW}
$$

Now it is clear that the mean-square error $\xi$ is a quadratic function of the weight vector W. When we expand this expression, the elements of $\mathrm{W}$ will appear in first and second degree only. If we have two weights, we can get the quadratic error function, or performance surface. ( the vertical axis represents the mean-square error and the horizontal axes the values of the two weights.) The point at the "bottom of the surface" is projected onto the weight-vector plane and then we can obtain the optimal weight vector $\mathrm{W}^{*}$ or the minimum mean-square error point. And there is only a single global optimum in this performance surface. To search the performance surface for minimum point, we can do gradient methods:

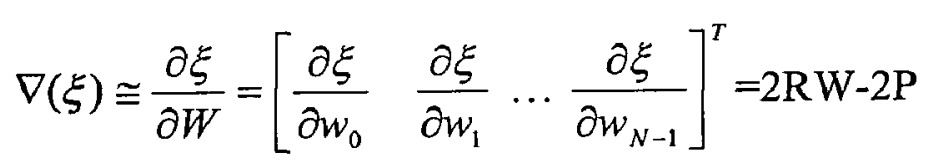

To obtain the minimum mean-square error, the weight vector $\mathrm{W}$ is set at its optimal value $\mathrm{W}^{*}$ and where the gradient is zero.

$$
\begin{aligned}
& \nabla=0=2 \mathrm{RW}^{*}-2 \mathrm{P} \\
& R \mathrm{~W}^{*}=\mathrm{P}
\end{aligned}
$$


Then the

$$
\mathrm{W}^{*}=\mathrm{R}^{-1} \mathrm{P}
$$

To develop the Least Mean Square (LMS) algorithm, we take $\varepsilon_{k}^{2}$ itself as an estimate of $\xi_{k}$. Then, for each iteration in the adaptive process, we have a gradient estimate of the form

$$
\nabla_{k}=\left[\begin{array}{l}
\frac{\partial \varepsilon_{k}^{2}}{\partial w_{0}} \\
\cdot \\
\cdot \frac{\partial \varepsilon_{k}^{2}}{\partial w_{N-1}}
\end{array}\right]=2 \varepsilon_{k}\left[\begin{array}{l}
\frac{\partial \varepsilon_{k}}{\partial w_{0}} \\
\cdot \\
\cdot \\
\frac{\partial \varepsilon_{k}}{\partial w_{N-1}}
\end{array}\right]=-2 \varepsilon_{k} \mathrm{X}_{k}
$$

With this simple estimate of the gradient, we can have

$$
\mathrm{W}_{k+1}=\mathrm{W}_{k}-\mu \nabla_{k}=\mathrm{W}_{k}+2 \mu \varepsilon_{k} \mathrm{X}_{k}
$$

This is the LMS algorithm. $\mu$ is the gain constant that regulates the speed and stability of adaptation. The LMS algorithm can be implemented in a practical system without squaring, averaging, or differentiation and is simple and efficient. For each iteration the LMS algorithm requires $2 \mathrm{~N}$ additions and $2 \mathrm{~N}+1$ multiplications $(\mathrm{N}$ is the filter length, $\mathrm{N}$ for calculating the output, one for $2 \mu \varepsilon_{k}$ and an additional $N$ for the scalar by vector multiplication). 


\subsection{NLMS Algorithms}

When $\mu$ is optimized as described by $\mu(n)=\frac{\alpha}{\beta+x_{M}^{T}(n) x(n)}, \alpha \in(0,2), 0 \leq \beta$,

$\beta$ guarantees that the denominator never becomes zero, while $\alpha$ is a relaxation factor, the normalized LMS algorithm results. In this case, $\mu$ is time varying.

The echo canceller coefficients $w_{k}(m)$ are adapted to minimize the energy of the echo signal on a telephone line. Assuming that the speech signals $x_{A}(m)$ and $x_{B}(m)$ are uncorrelated, the energy on the telephone line from B to A is minimized when the echo canceller output $\hat{x}_{A}$ echo $(m)$ is equal to the echo $\hat{x}_{B} \quad(m)$ on the line. The echo canceller coefficients can be adapted using the least mean squared error (LMS) adaptation algorithm or one of the most widely used algorithms for adaptation of the coefficients of an echo canceller is the normalized least mean square error(NLMS) method. The timeupdated equation describing the adaptation of the filter coefficient vector is

$$
w(m)=w(m-1)+\mu \frac{e(m)}{x(m)_{A}^{T} x_{A}(m)} x_{A}(m)
$$

where $x_{A}(m)=\left[x_{A}(m), \ldots, x_{A}(m-N+1)\right]$ and $x(m)=\left[x_{0}(m), \ldots, x_{P-1}(m-N+1)\right]$ are the input signal vector and the coefficient vector of the echo canceller, and $e(m)$ is the difference between the signal and the echo line and the output of the echo synthesizer. The normalized quanity $x(m){ }_{A}^{T} x_{A}(m)$ is the energy of the input speech to the adaptive filter. The scalar $\mu$ is the adaptation step size, and controls the speed of convergence, the steady-state error and the stability of the adaptation process.

Each iteration of the NLMS algorithm requires $3 \mathrm{~N}+1$ multiplications ( $\mathrm{N}$ is filter length), this is only N more than the standard LMS algorithm. 


\subsection{Fast Block LMS Algorithm}

In the normalized LMS algorithm, the filter coefficients of a finite-duration impulse (FIR) filter are adapted in the time domain. Because the Fourier transform maps timedomain signals into the frequency domain and the inverse Fourier transform provides the inverse mapping that takes the signals back into the time domain, it is workable to perform the adaptation of filter coefficients in the frequency domain, which is called frequency-domain adaptive filtering.

In a block-adaptive filter, shown in Figure 2.1, the input data sequence $\mathrm{x}(\mathrm{n})$ is sectioned into $L$-point blocks by means of a serial-to-parallel converter, and the blocks of input data so produced are applied to an FIR filter of length $M$, one block at a time. The coefficients of the filter are updated after the collection of each block of data samples, so that adaptation of the filter proceeds on a block-by-block basis rather than on a sampleby-sample basis as in the conventional LMS algorithm.

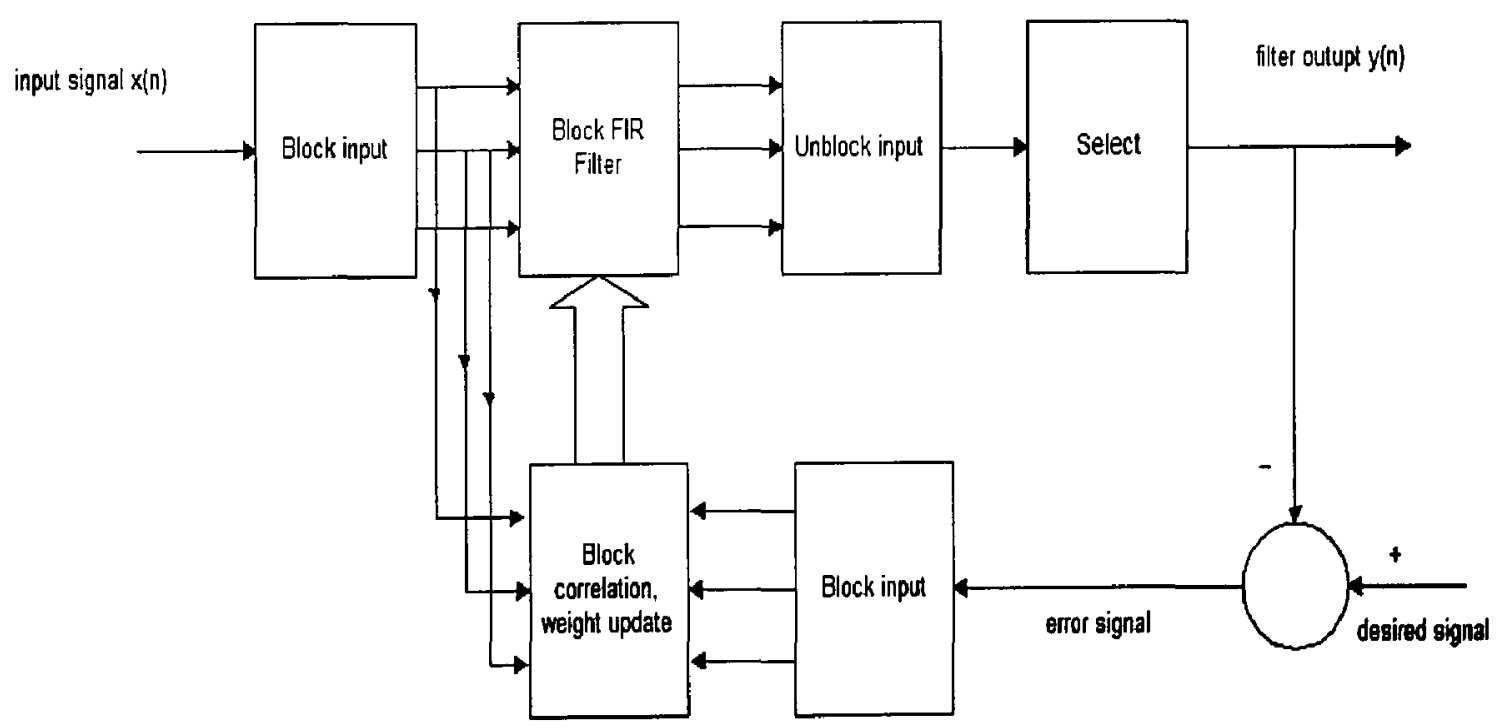

Figure 2.1 Block-adaptive filter 
The Fast Block LMS algorithm represents a precise frequency-domain implementation of the block LMS algorithm and its convergence properties are identical to those of the block LMS algorithm.

Fast Block LMS Algorithm is based on Overlap-Save Sectioning (Assuming RealValued Data).

Initialization:

$$
\begin{aligned}
& \hat{w}(0)=2 \mathrm{M} \text { by } 1 \text { zero vector } \\
& \operatorname{Pi}(0)=\delta_{i}
\end{aligned}
$$

where the $\delta_{i}$ are small positive constants and $i=0, \ldots, 2 M-1$

Notations:

$$
\begin{array}{ll}
0 & =\mathrm{M} \text { by } 1 \text { zero vector } \\
\mathrm{FFT} & =\text { fast Fourier transformation } \\
\mathrm{IFFT} & =\text { inverse fast Fourier transformation } \\
\alpha & =\text { adaptation constant }
\end{array}
$$

Computation: For each new block of $M$ input samples, compute:

Filtering

$$
\begin{aligned}
& U(k)=\operatorname{diag}\left\{F F T[u(k M-M), \ldots, u(k M-1), u(k M), \ldots, u(k M+M-1)]^{T}\right\} \\
& y^{T}(k)=\text { the last } M \text { elements of IFFT }[U(k) \hat{W}(k)]
\end{aligned}
$$

Error estimation

$$
\begin{gathered}
e(k)=d(k)-y(k) \\
E(k)=F F T\left[\begin{array}{c}
0 \\
e(k)
\end{array}\right]
\end{gathered}
$$

Signal-power estimation

$$
\begin{aligned}
& P i(k)=\gamma P i(k-1)+(1-\gamma)|U i(k)|^{2} \quad, \quad i=0,1, \ldots ., 2 M-1 \\
& D(k)=\operatorname{diag}\left[P_{0}^{-1}(k), P_{1}^{-1}(k), \ldots, P_{2 M-1}{ }^{-1}(k)\right]
\end{aligned}
$$

Filter coefficient adaptation

$$
\begin{aligned}
& \phi(k)=\text { the first } M \text { elements of } \operatorname{IFFT}\left[D(k) U^{H}(k) E(k)\right] \\
& \hat{W}(k+1)=\hat{W}(k)+\alpha F F T\left[\begin{array}{c}
\phi(k) \\
0
\end{array}\right]
\end{aligned}
$$




\section{Choice of Block Size:}

The block size $\mathrm{L}$ in relation to the length $\mathrm{M}$ of the adaptive filter. There are three possible choices that can be chosen, each with its own practical implications:

- $L=M$ which is optimum choice

- $L<M \quad$ advantage of reduced processing delay and if the block size smaller than the filter length, the adaptive filtering algorithm computationally is still more efficient than the conventional LMS algorithm.

- $L>M$ increased redundant operations in the adaptive process, because the estimation of the gradient vector now uses more information than the filter itself.

In the fast block LMS algorithm there are 5 FFT transforms, requiring approximately $2 \mathrm{M} \log (2 \mathrm{M})$ real multiplications each, and also other $16 \mathrm{M}$ operations (when updating the parameters, computing the errors, element-wise multiplications of FFT transformed vectors), so the total is $10 \mathrm{M} \log (2 \mathrm{M})+16 \mathrm{M}=10 \mathrm{M} \log (\mathrm{M})+26 \mathrm{M}$. 


\subsection{RLS algorithm}

The Recursive Least Square (RLS) algorithm is used to minimize the cost function:

$$
\zeta=\sum_{k=1}^{n} \lambda^{n-k} e_{n}^{2}(k)
$$

Where $\mathrm{k}=1$ is the time at which the RLS algorithm starts and $\lambda$ is a small positive constant very close to, but smaller than 1 .

Unlike the LMS algorithm and its derivatives, the RLS algorithm directly considers the values of previous error estimations. In fact, it is impossible to use all previous values of the estimation error from the start of the algorithm with the cost equation in real FIR implementation for the computation complexity. In practice, only a finite number of previous values are considered, this number corresponds to the order of the RLS FIR filter, $N$.

$$
\begin{aligned}
& y_{n}(k)=w^{T}(n) x(k), e_{n}(k)=d(k)-y_{n}(k) \\
& d(n)=[d(1), d(2) \ldots d(n)]^{T}, e(n)=\left[e_{n}(1), e_{n}(2) \ldots e_{n}(n)\right]^{T} \\
& e(n)=d(n)-y(n) \\
& \zeta=\sum_{k=1}^{n} \lambda^{n-k} e_{n}^{2}(k)=e^{T}(n) \tilde{\Lambda} e(n)=d^{T} \tilde{\Lambda} d-2 \tilde{\theta_{\lambda}^{T}} w+w^{T} \tilde{\Phi_{\lambda}} w
\end{aligned}
$$

where $\tilde{\theta}_{\lambda}(n)=x(n) \tilde{\Lambda} d(n), \tilde{\Phi}_{\lambda}(n)=x(n) \tilde{\Lambda}(n) x^{T}(n)$

$$
\bar{w}(n)=\tilde{\Phi}_{\lambda}^{-1}(n) \tilde{\theta}_{\lambda}(n)
$$

Find the inverse matrix using recursive form,

$$
\tilde{\Phi}_{\lambda}^{-1}(n)=\lambda \tilde{\Phi}_{\lambda}^{-1}(n-1)+x(n) x^{T}(n)=\lambda^{-1}\left(\tilde{\Phi}_{\lambda}^{-1}(n-1)-k(n) x^{T}(n) \tilde{\Phi}_{\lambda}^{-1}(n-1)\right)
$$




$$
\begin{aligned}
& \text { where } k(n)=\frac{\lambda^{-1} \Phi_{\lambda}^{-1}(n-1) x(n)}{1+\lambda^{-1} x^{T}(n) \Phi_{\lambda}^{-1}(n-1) x(n)}=\frac{1}{\lambda+x^{T}(n) u(n)} u(n) \\
& \tilde{\theta}_{\lambda}(n)=\lambda \tilde{\theta}_{\lambda}(n-1)+x(n) d(n)
\end{aligned}
$$

so RLS algorithm filter weight update vector

$$
\begin{aligned}
& \bar{w}(n)=\tilde{\Phi}_{\lambda}^{-1}(n) \tilde{\theta}_{\lambda}(n) \\
& \left.=\tilde{\Phi}_{\lambda}^{-1}(n-1) \tilde{\theta}_{\lambda}(n-1)-k(n) x^{T} \tilde{\Phi}_{\lambda}^{-1}(n-1)\right) \tilde{\theta}_{\lambda}(n-1)+k(n) d(n) \\
& =\bar{w}(n-1)+k(n) \bar{e}_{n-1}(n)
\end{aligned}
$$

where $\quad \bar{e}_{n-1}(n)=d(n)-\bar{w}^{T}(n-1) x(n)$

Each iteration of the RLS algorithm requires $4 \mathrm{~N}^{\wedge} 2$ multiplication operations $(\mathrm{N}$ is the filter length). 


\section{Chapter 3}

\section{Double Talk Detection Algorithms}

During double-talking periods, there exists the other end speaker's adaptive signal $v(n)$ which acts as a very large interference to the adaptive filter. If the adaptive filter continues to adjust its coefficients during double-talking periods, the adaptive filter will be greatly disturbed and will quickly diverge from its convergence state. Therefore, double-talk detectors are used in adaptive voice echo cancellers to detect the doubletalking periods, and the adaptive filter coefficients adjustment is prohibited during these periods to prevent the echo canceller from being disturbed by the other end speaker's signal. Double-talk detection plays a very important part in adaptive acoustic echo cancellation. The basic requirement for a double-talk detector is that it can detect doubletalking quickly and accurately and it should also have the ability to distinguish the double-talking conditions from echo path variations and quickly track variations in the echo path.

\subsection{The Geigel algorithm}

A simple approach is to measure the power of the received signal and compare it to the power of the far-end signal; as shown in Figure 1.1, $z(t)$ is the received signal, $x(t)$ is the far end signal, this is the Geigel algorithm [4] and the decision variable is defined as $d_{G}(t)=\frac{|z(t)|}{\max \{|x(t)|, \ldots, \mid x(t-n+1)\}}$. 
If $d_{G}(t)$ is larger than some preset threshold, $T_{G}$, it is treated that Double Talk is occurring, otherwise not. The Geigel detector is computationally simple and need little memory, but the choice of $T_{G}$ is not easy to obtain good performance.

\subsection{Normalized cross-correlation algorithm}

As shown in Figure 1.1, the power of the received signal can be written as

$$
\sigma_{z}^{2}(t)=h_{t}^{T} R_{x}(t) h_{t}+\sigma_{v}^{2}(t)
$$

where $R_{x}(t)=E\left\{x_{t} x_{t}^{T}\right\}$ is the $L \times L$ covariance matrix of the far-end signal,

$\sigma_{v}^{2}(t)$ is the power of the near-end signal,

$\sigma_{z}^{2}(t)$ is the power of the received signal,

$h_{t}$ is room acoustic response.

As $y(t)=h_{t}^{T} x_{t}$,

Then $r_{x y}(t)=E\left\{x_{t} y(t)\right\}=R_{x}(t) h_{t}$,

Yielding,

$$
h_{t}=R_{x}^{-1}(t) r_{x y}(t)
$$

So,

$$
\sigma_{z}^{2}(t)=r_{x y}^{T}(t) R_{x}^{-1}(t) r_{x y}(t)+\sigma_{v}^{2}(t)
$$

When there is no near-end signal is present, $v(t)=0$, then $z(t)=y(t)$ and

$$
\sigma_{z}^{2}(t)=r_{x y}^{T}(t) R_{x}^{-1}(t) r_{x y}(t), \quad \text { with } r_{x z}(t)=E\left\{x_{t} z(t)\right\}
$$

The detection variable is $d(t)=\frac{r_{x z}^{T}(t) R_{x}^{-1} r_{x z}(t)}{\sigma_{z}^{2}(t)}=\frac{r_{x z}^{T}(t) h}{\sigma_{z}^{2}(t)}$, 
where $h$ is the estimated room acoustic response and $r_{x z}(t)$ is the estimated crosscorrelation between the far-end signal and the received signal.

The nominator is the power of the received signal if no near-end signal is present. The denominator is the actual power of the received signal. Thus, if no near end signal is present, $d(t) \approx 1$, otherwise $d(t)<1$. The Double Talk decision is formed as $d(t)<T_{n}$, double talk present, otherwise not.

When there is no near-end talk, it is known that the decision variable $d$ is $=1$ for $v=0$ and $d<1$ for $\mathrm{v} \neq 0$ when there exist near-end talk. To calculate the decision variable $d$, in implementation, we use the following smoothed estimate:

$$
\begin{aligned}
& r_{x z}(t)=\alpha r_{x z}(t-1)+(1-\alpha) X(t) z(t) \\
& \sigma_{z}^{2}(t)=\alpha \sigma_{z}^{2}(t-1)+(1-\alpha) z(t)^{2}
\end{aligned}
$$

where $\alpha$ is a smoothing factor which lies in $(0,1)$. In experiment, $\alpha$ is set to be 0.9 and

$$
r_{x z}(t)=\sum_{k=0}^{L-1} X(t-k) z(t-k)
$$

using a L length sliding window, the $r_{x z}(t)$ can be estimated [5] [6][7][8]. 


\subsection{Evaluation procedure of double detectors}

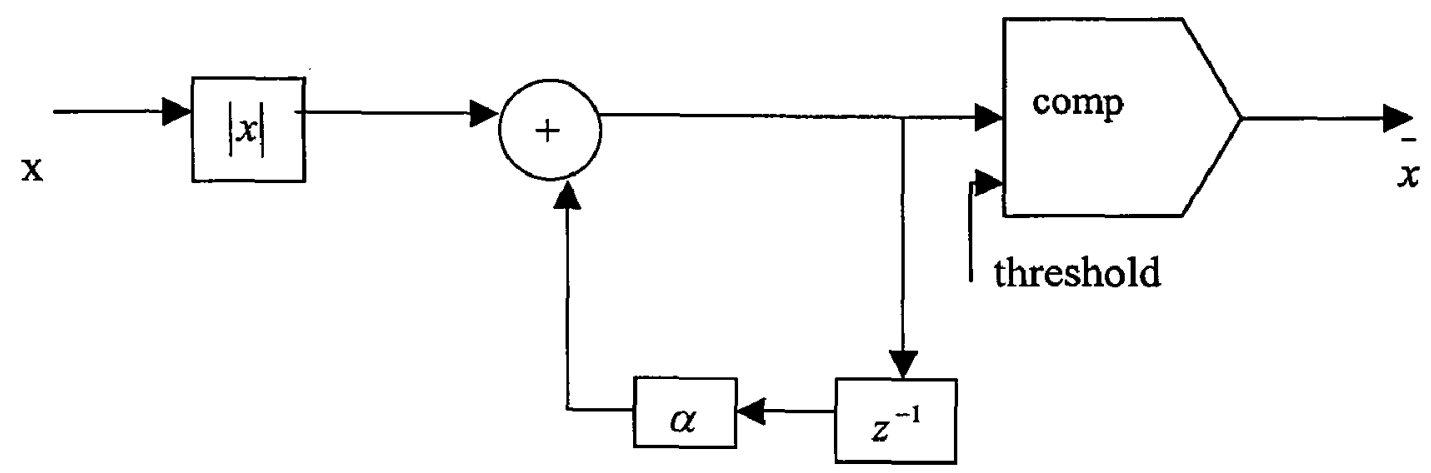

Figure 3.1 Voice activity detector

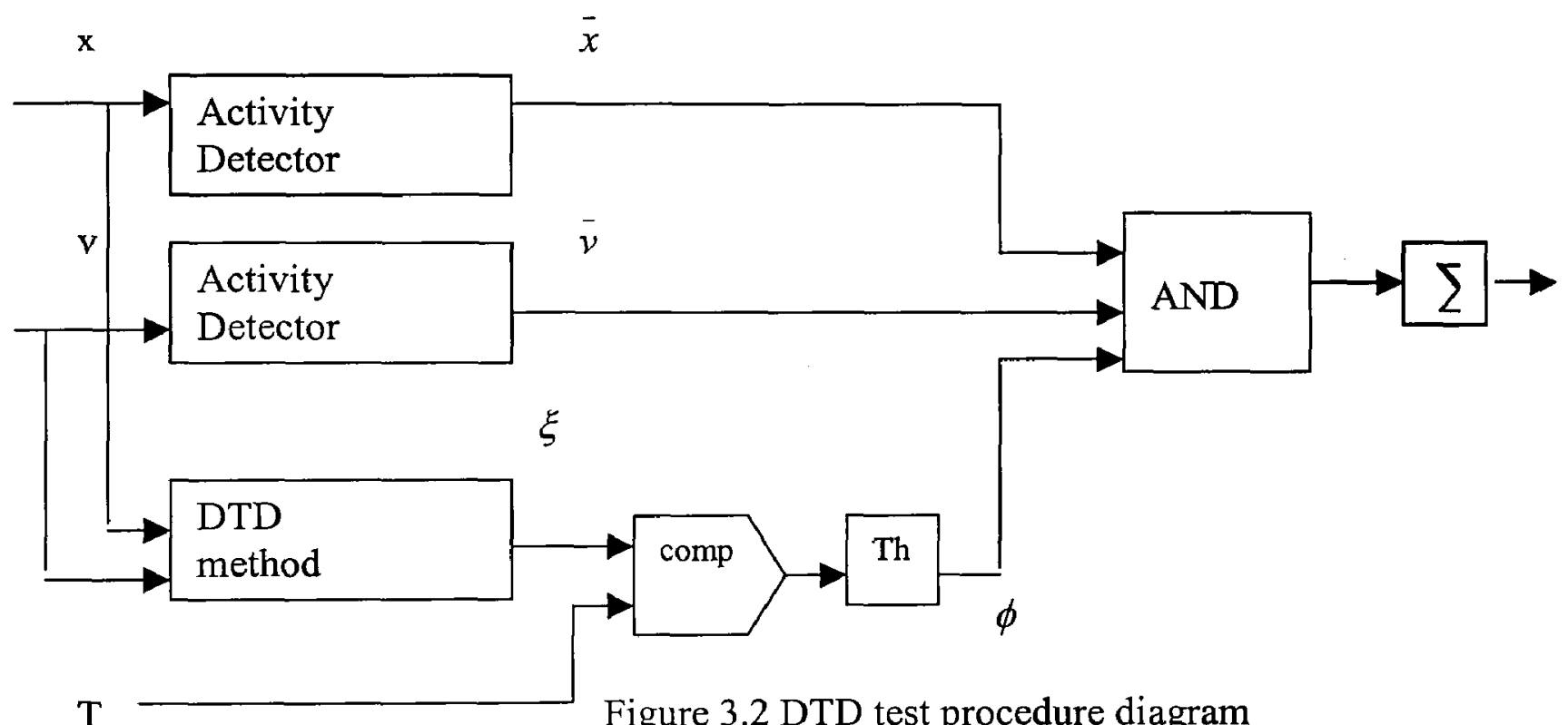

In the DTD methods discussed in the previous section, the role of threshold $\mathrm{T}$ is essential to the performance. However, there hasn't been a systemic approach to select the value of T. In the paper [6][9][10], an objective technique is proposed for evaluating doubletalk detectors in acoustic echo cancellers. It view DTD as a binary detection problem and use it in actual operating environments. The general characteristics of a binary detection scheme are as follows. 
Probability of False Alarm $\left(P_{f}\right)$ : Probability of declaring detection when a target is not present.

Probability of Detection ( $\left.P_{d}\right)$ : Probability of successful detection when a target is present.

Probability of Miss ( $\left.P_{m}=1-P_{d}\right)$ : Probability of detection failure when a target is present.

$$
\begin{aligned}
& P_{f}=\frac{\sum \phi \bullet x}{N} \\
& P_{d}=\sum \phi \bullet \bar{x} \cdot \bar{v} / \sum \bar{x} \bullet \bar{v} \\
& P_{m}=1-\sum \phi \cdot \bar{x} \bullet \bar{v} / \sum \bar{x} \bullet \bar{v}
\end{aligned}
$$




\section{Chapter 4}

\section{Matlab Simulation \& Analysis}

\subsection{Adaptive algorithms}

Graphical User Interface

In this project, the command 'guide' is used in MATLAB v6.5 to make the Graphical

User Interface (GUI) of the AEC Analysis and Design program to help study different

AEC algorithms. The GUI of the program can be seen in Figure 4.1.

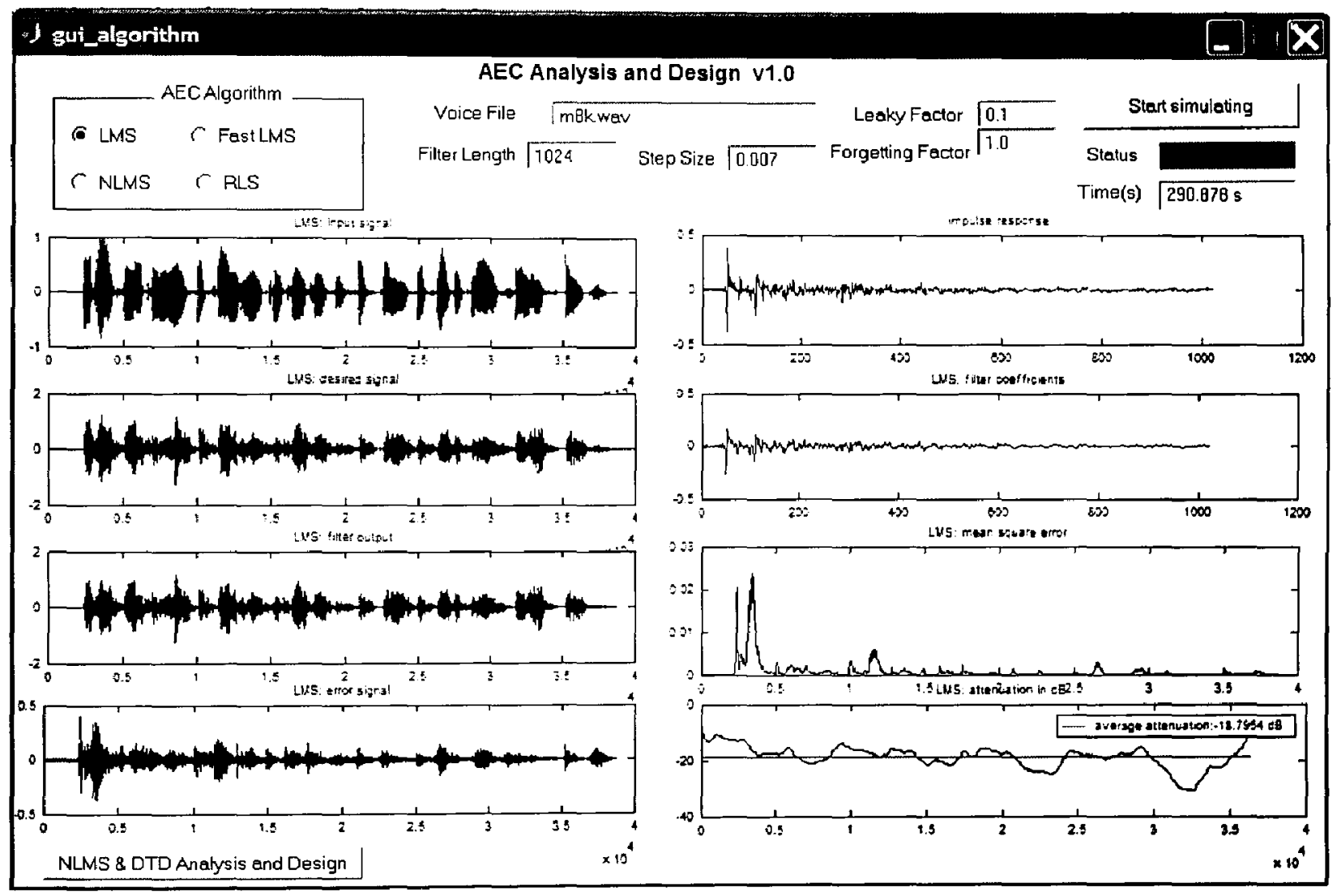

Figure 4.1 AEC Analysis and Design program GUI

In this program, user can choose:

1. AEC Algorithm

a. LMS algorithm

b. NLMS Algorithm 


\section{c. Fast LMS Algorithm}

2. Input signal file (wav format)

3. Filter Length

4. Step Size

5. Leaky Factor

6. Forgetting Factor

\subsubsection{LMS}

The Figure 4.2 shows the desired signal, adaptive output signal, estimation error and mean square error for the LMS algorithm with FIR Filter length of 1000, step size of 0.007 .
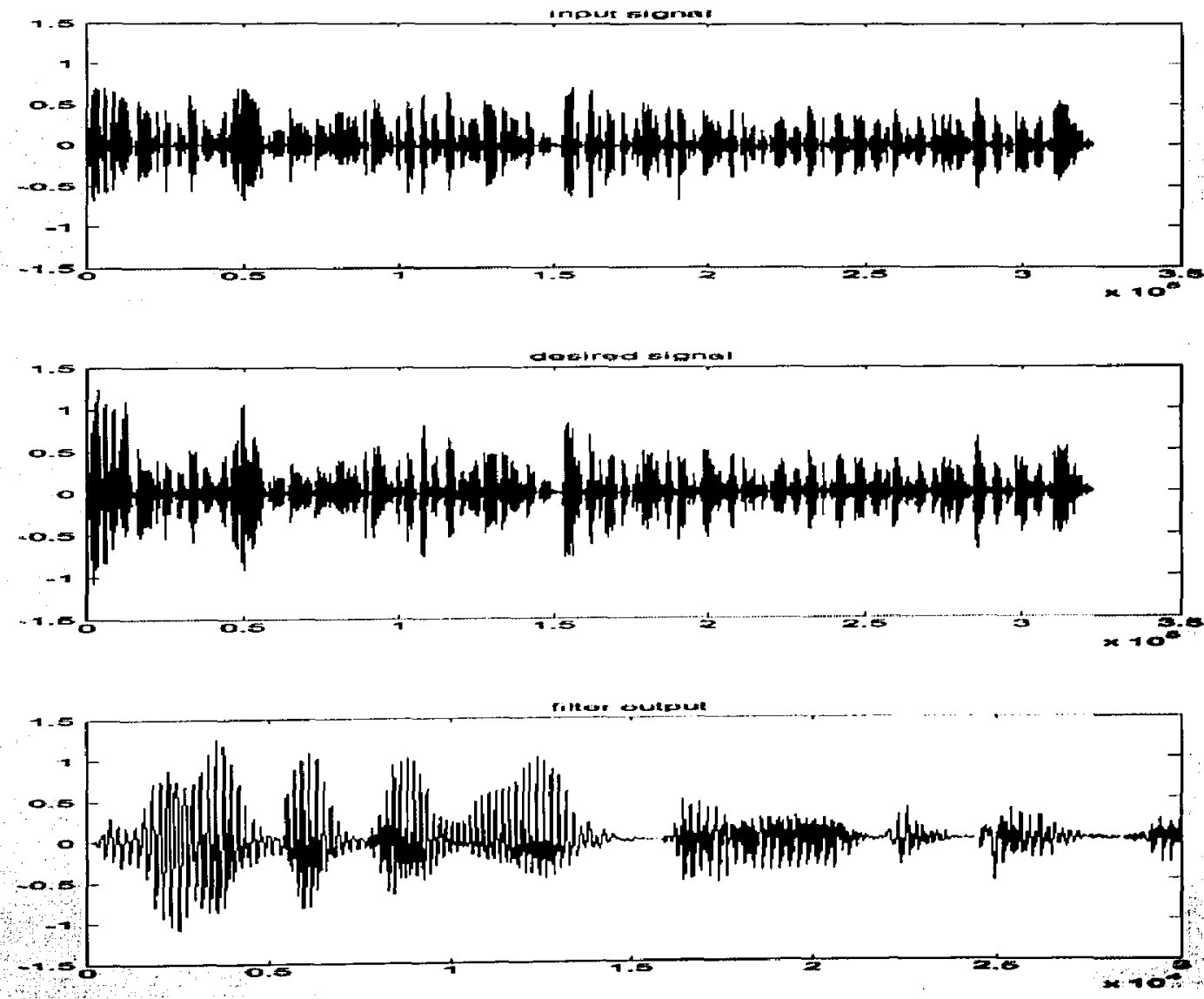

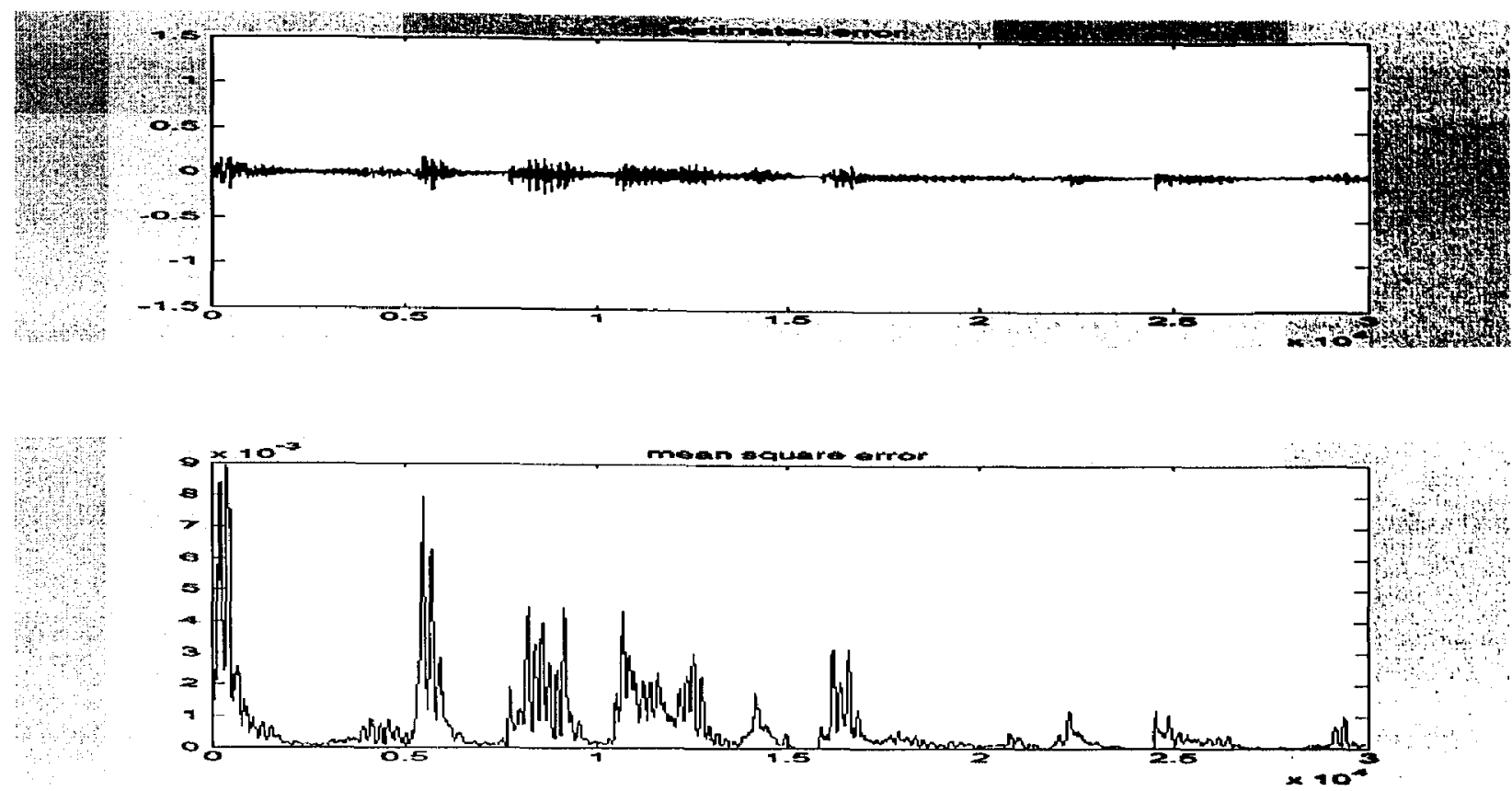

Figure 4.2 LMS algorithm
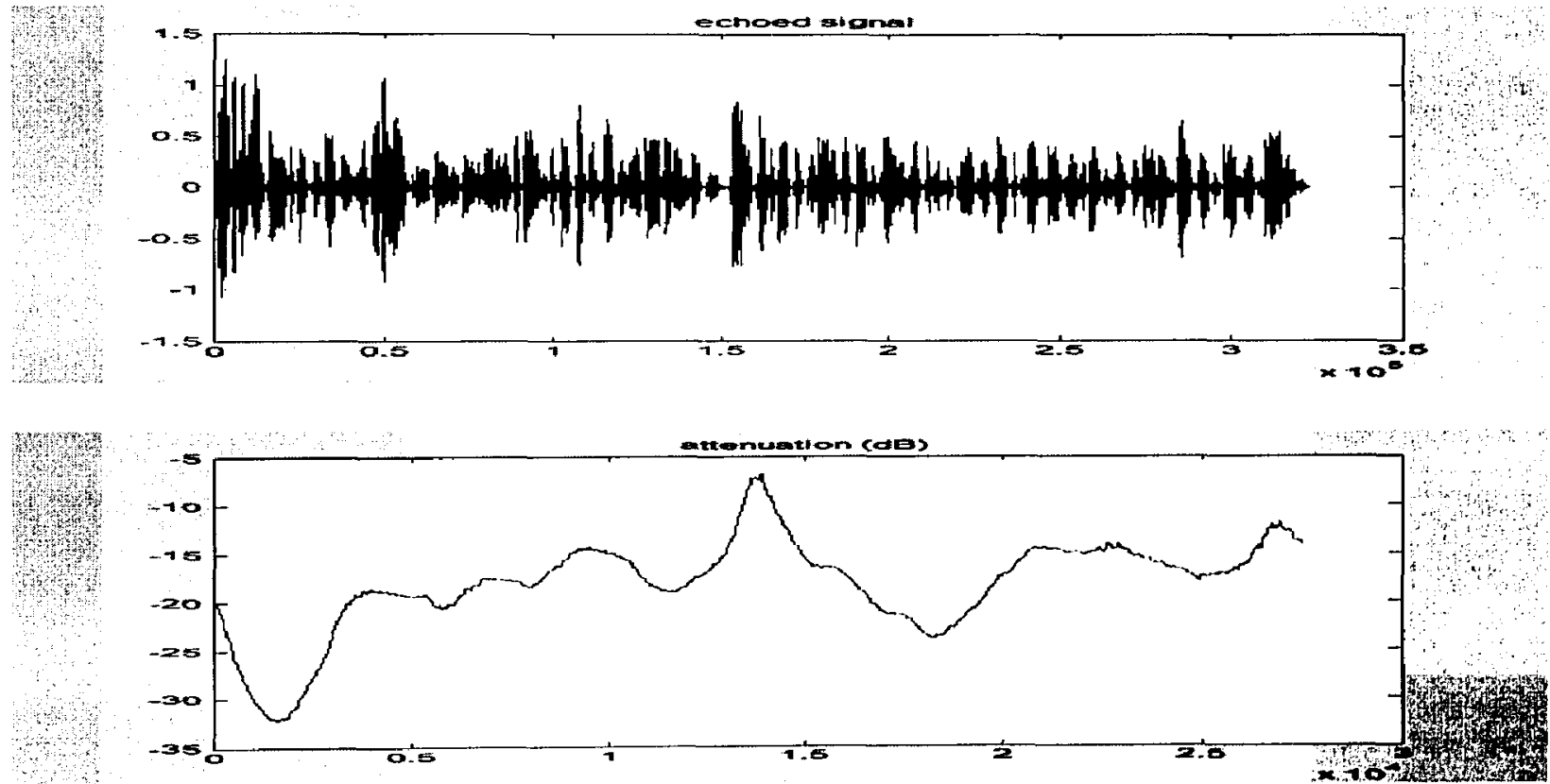

Figure 4.3 LMS echo signal attenuation

The average attenuation for LMS FIR filter is $-18.16 \mathrm{~dB}$.

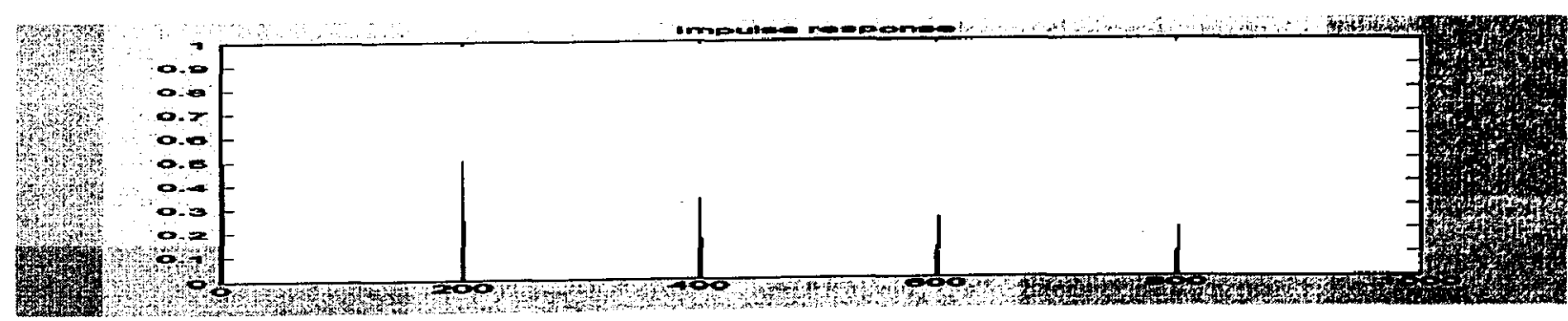




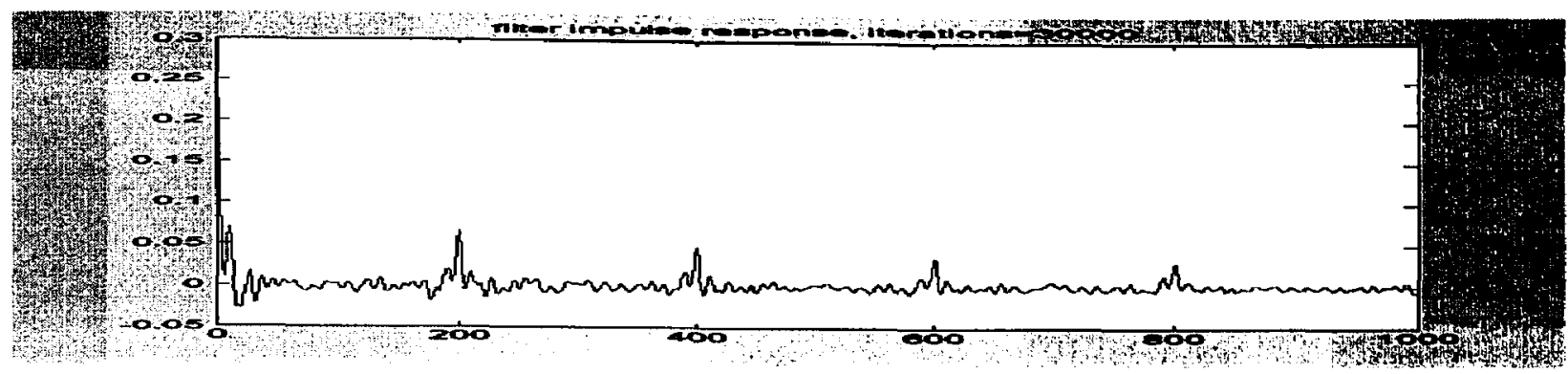

Figure 4.4 LMS FIR filter impulse response

\subsubsection{NLMS}

The Figure 4.5 shows the desired signal, filter output signal, estimation error and mean square error for the NLMS algorithm with FIR Filter length of 1000.
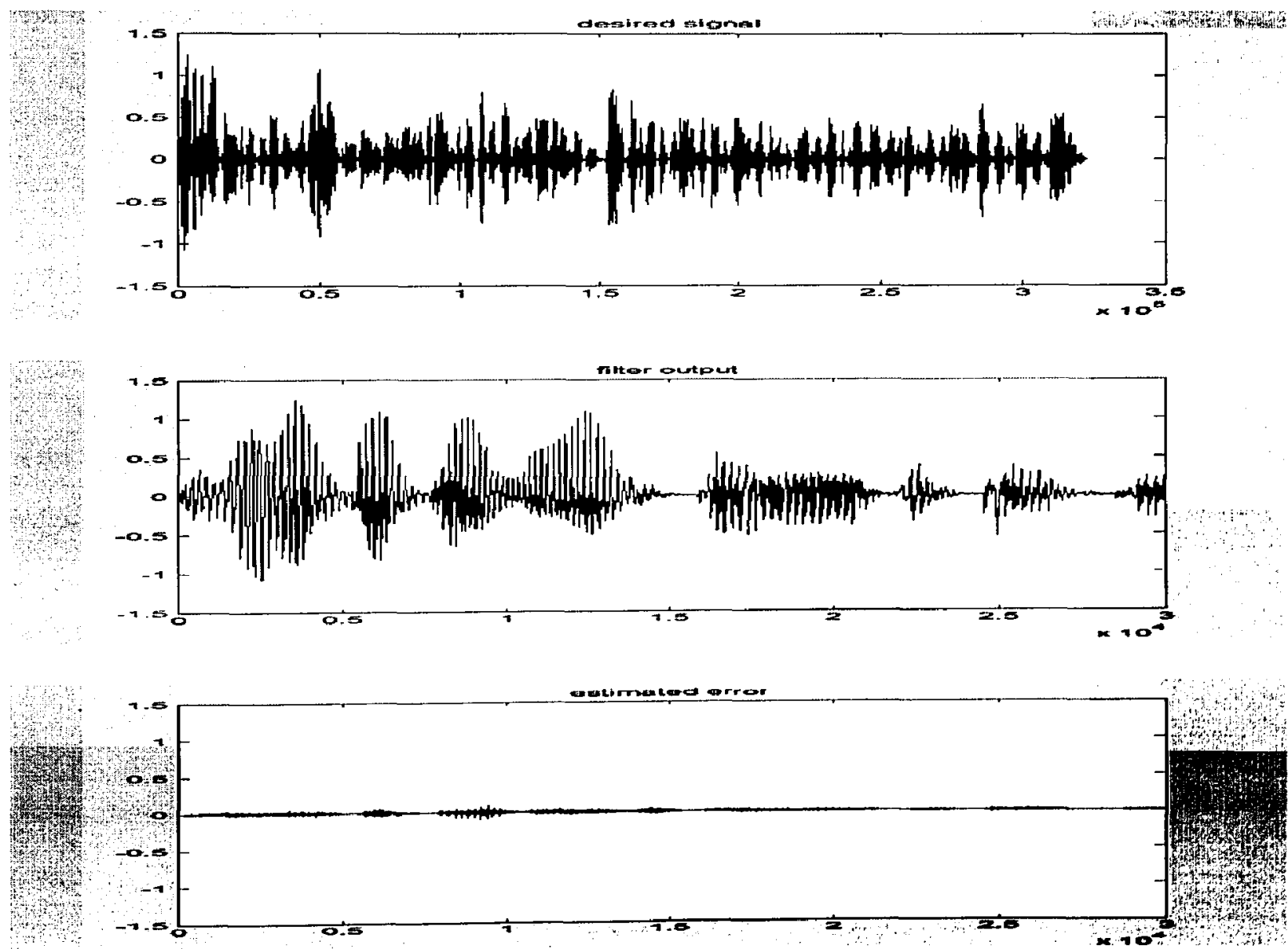

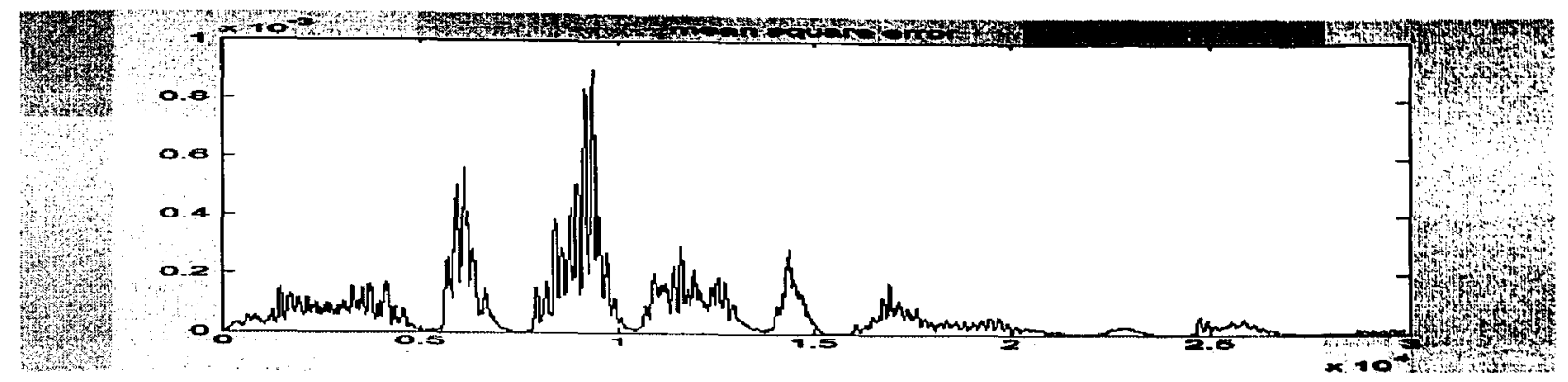

Figure 4.5 NLMS algorithm
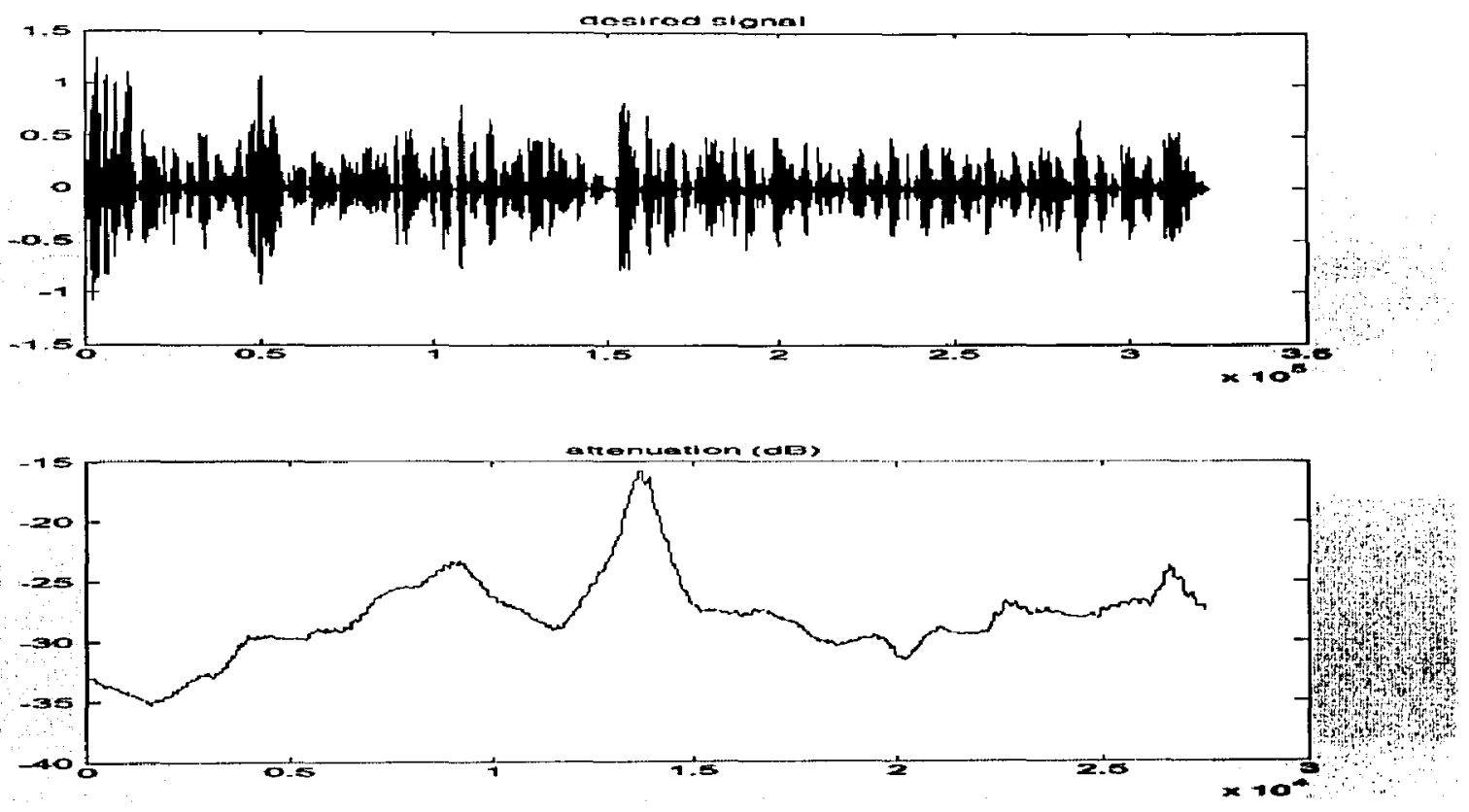

Figure 4.6 NLMS echo signal attenuation

The average attenuation for NLMS FIR filter is $-27.99 \mathrm{~dB}$

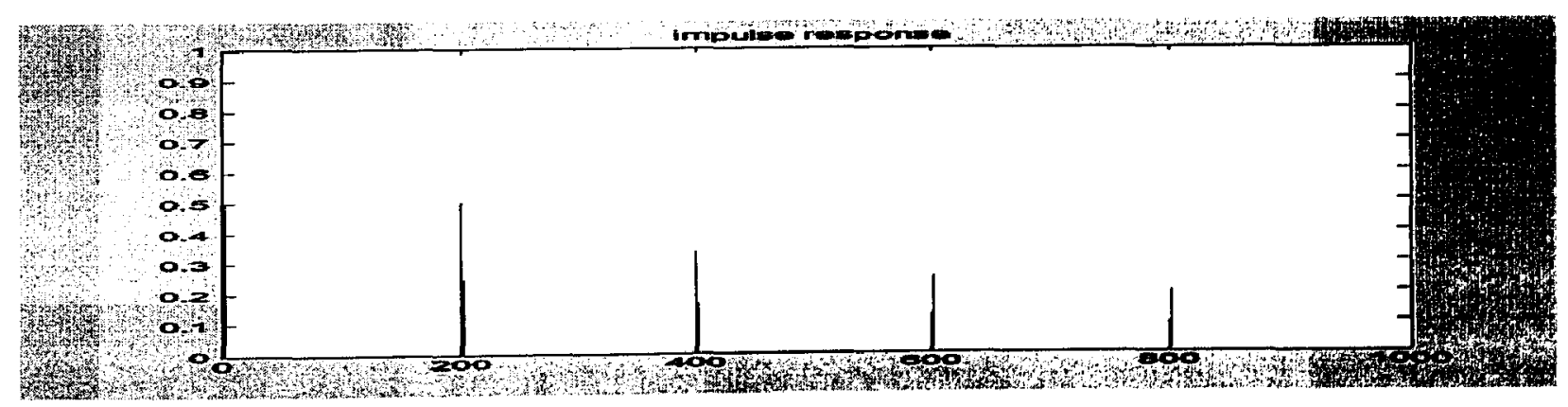



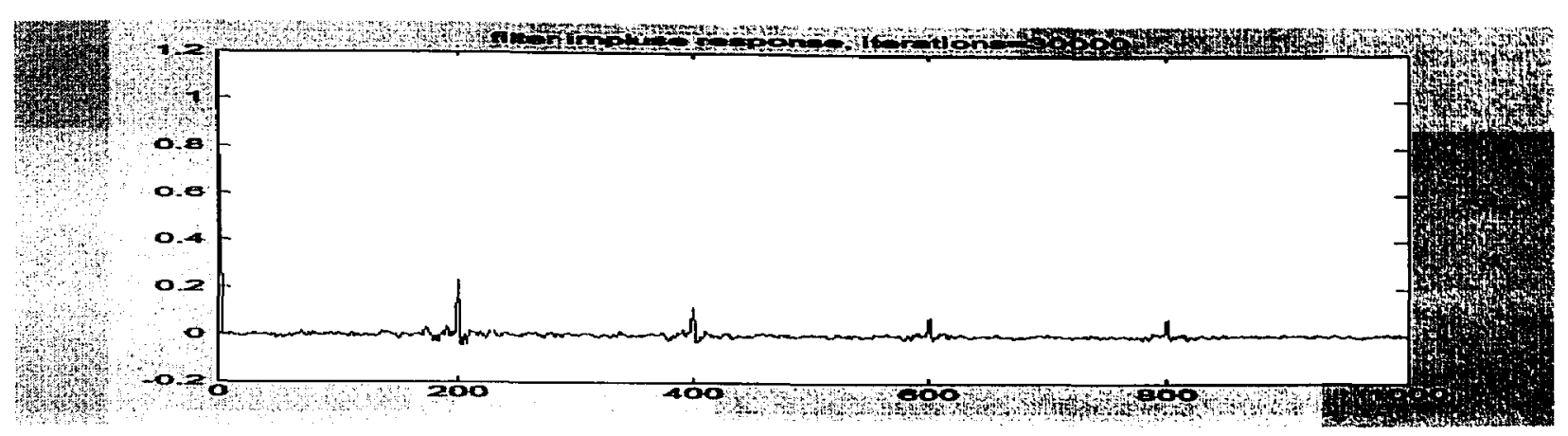

Figure 4.7 NLMS FIR filter impulse response

\subsubsection{Fast Block LMS}

The Figure 4.8 shows input signal, the desired signal, filter estimation error and mean square error for the FLMS algorithm with FIR Filter length of 1000.
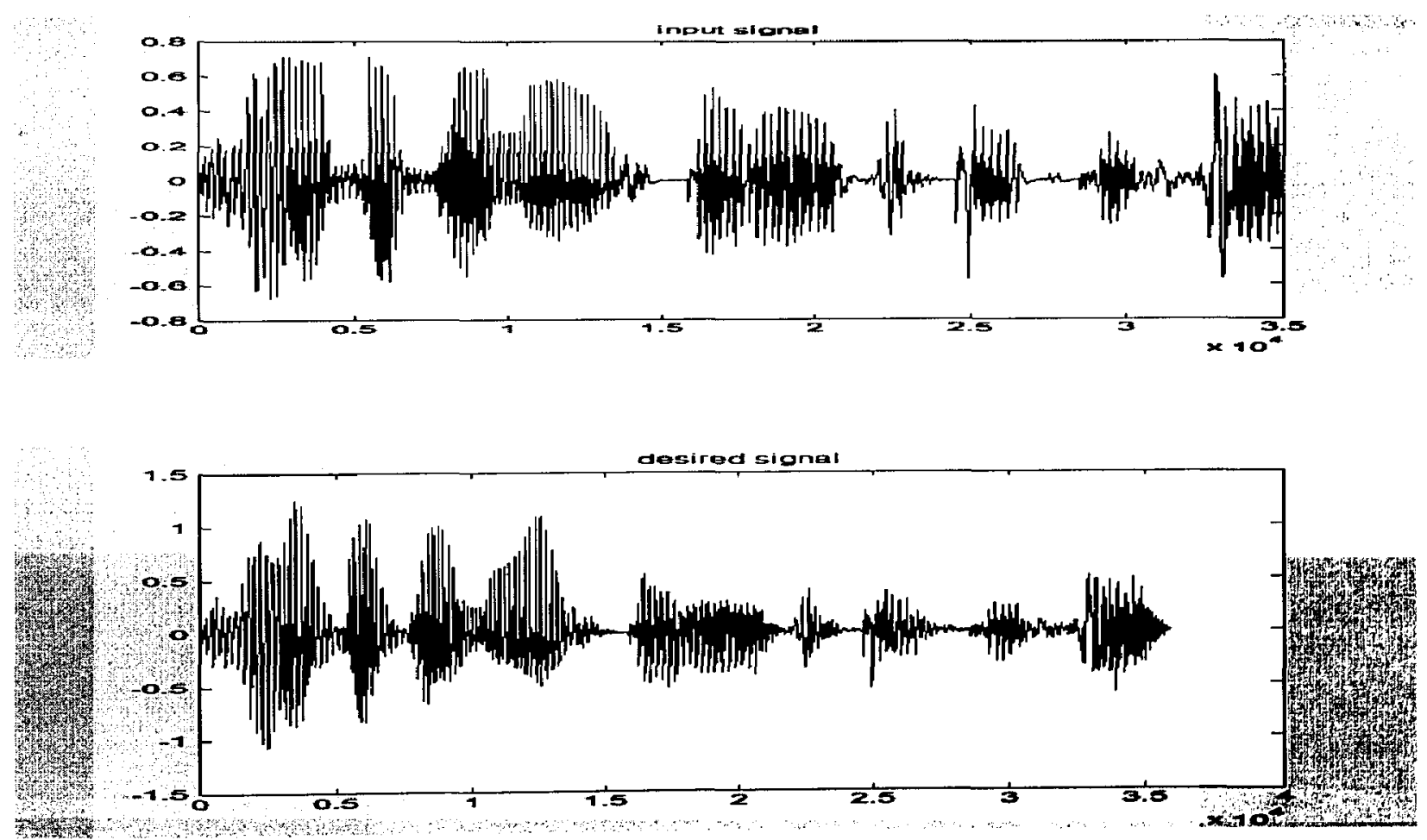

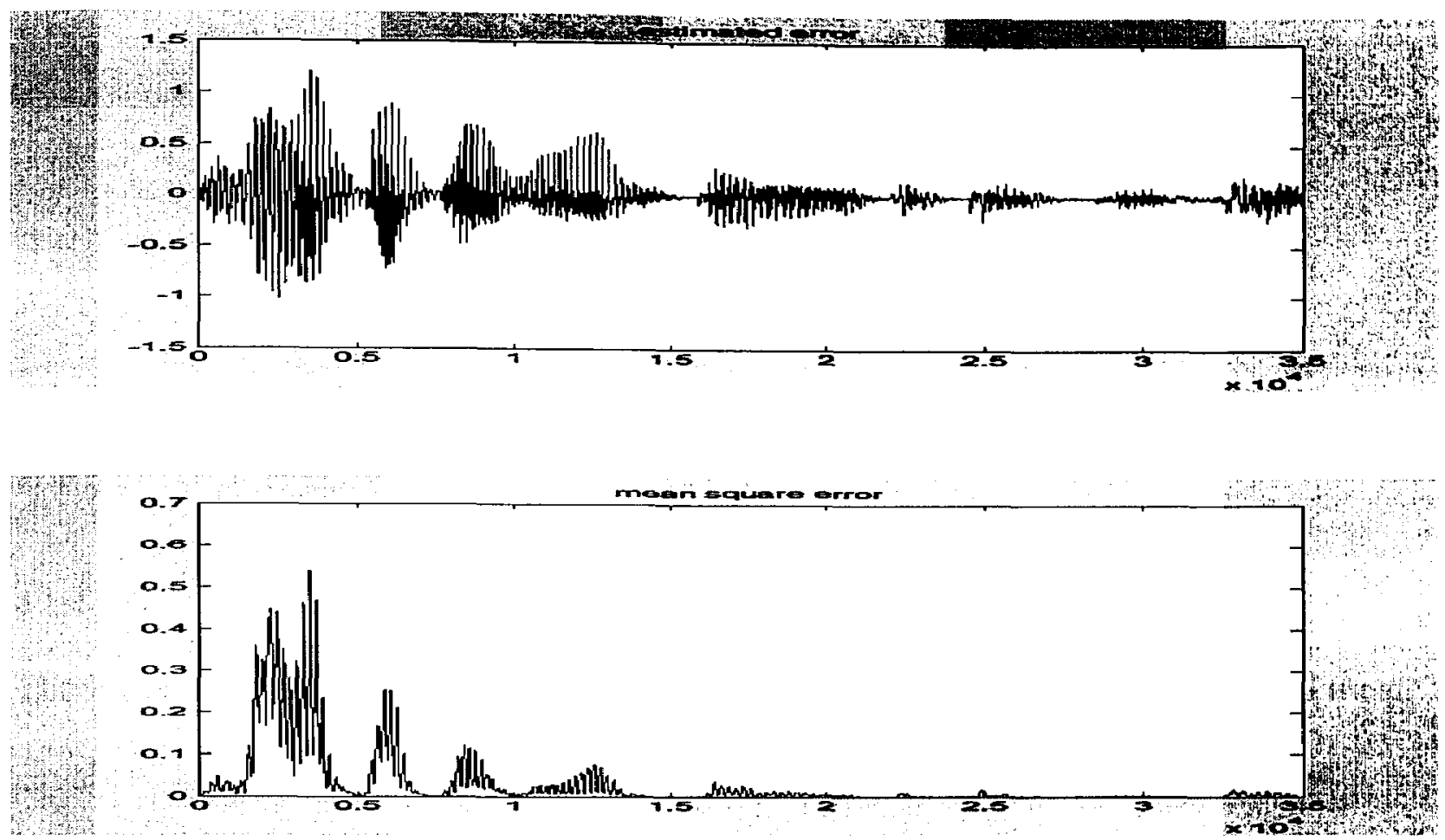

Figure 4.8 Fast Block LMS algorithm
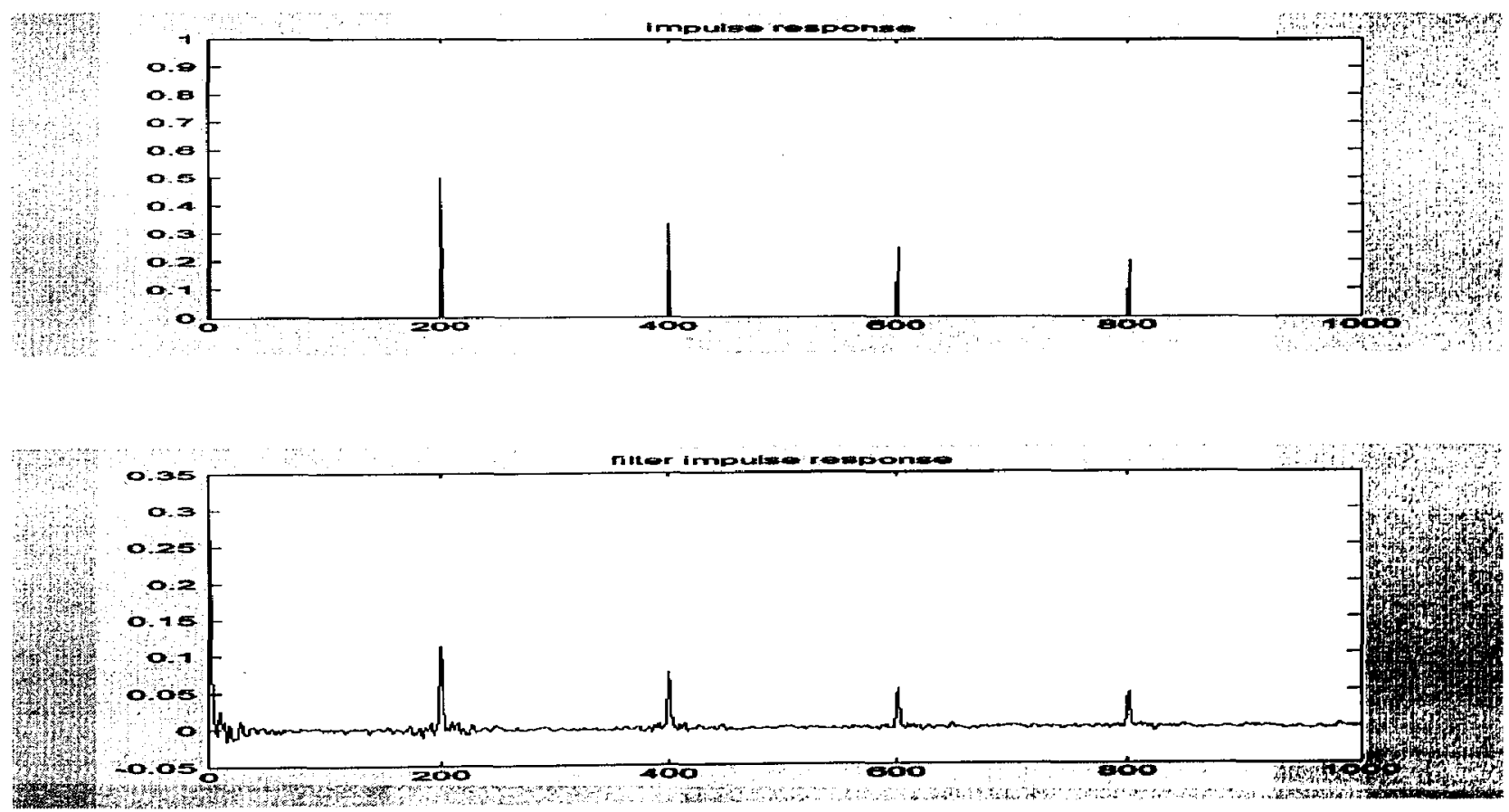

Figure 4.9 Fast Block LMS FIR filter impulse response 

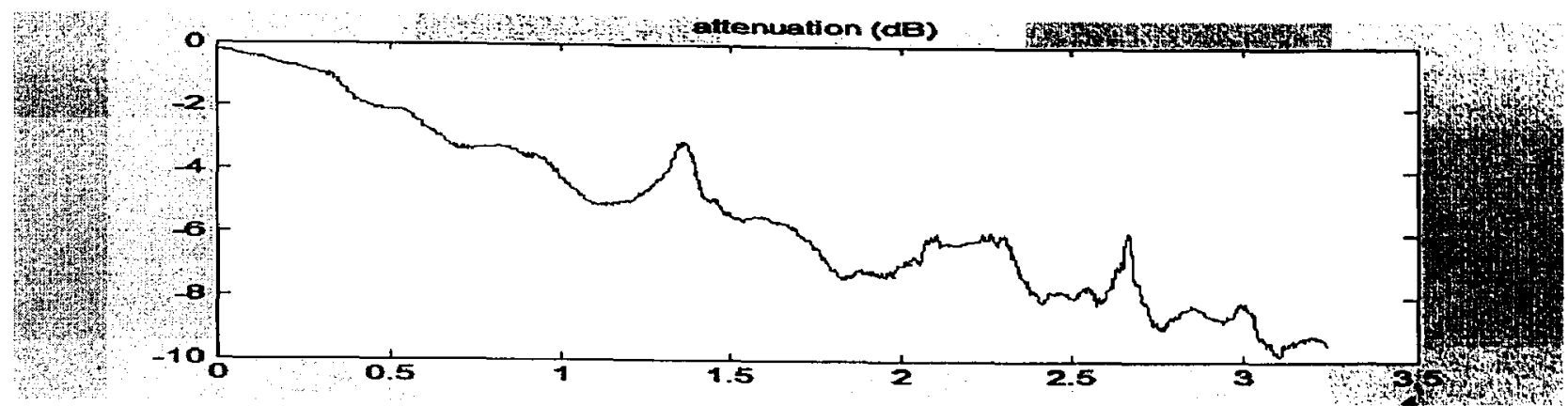

Figure 4.10 Fast Block LMS FIR filter echo signal attenuation

The average attenuation for NLMS FIR filter is $-5.33 \mathrm{~dB}$.

\subsubsection{RLS}

The Figure 4.11 shows the desired signal, filter output signal, estimation error and mean square error for the RLS algorithm with FIR Filter length of 1000 and $\lambda=1$.
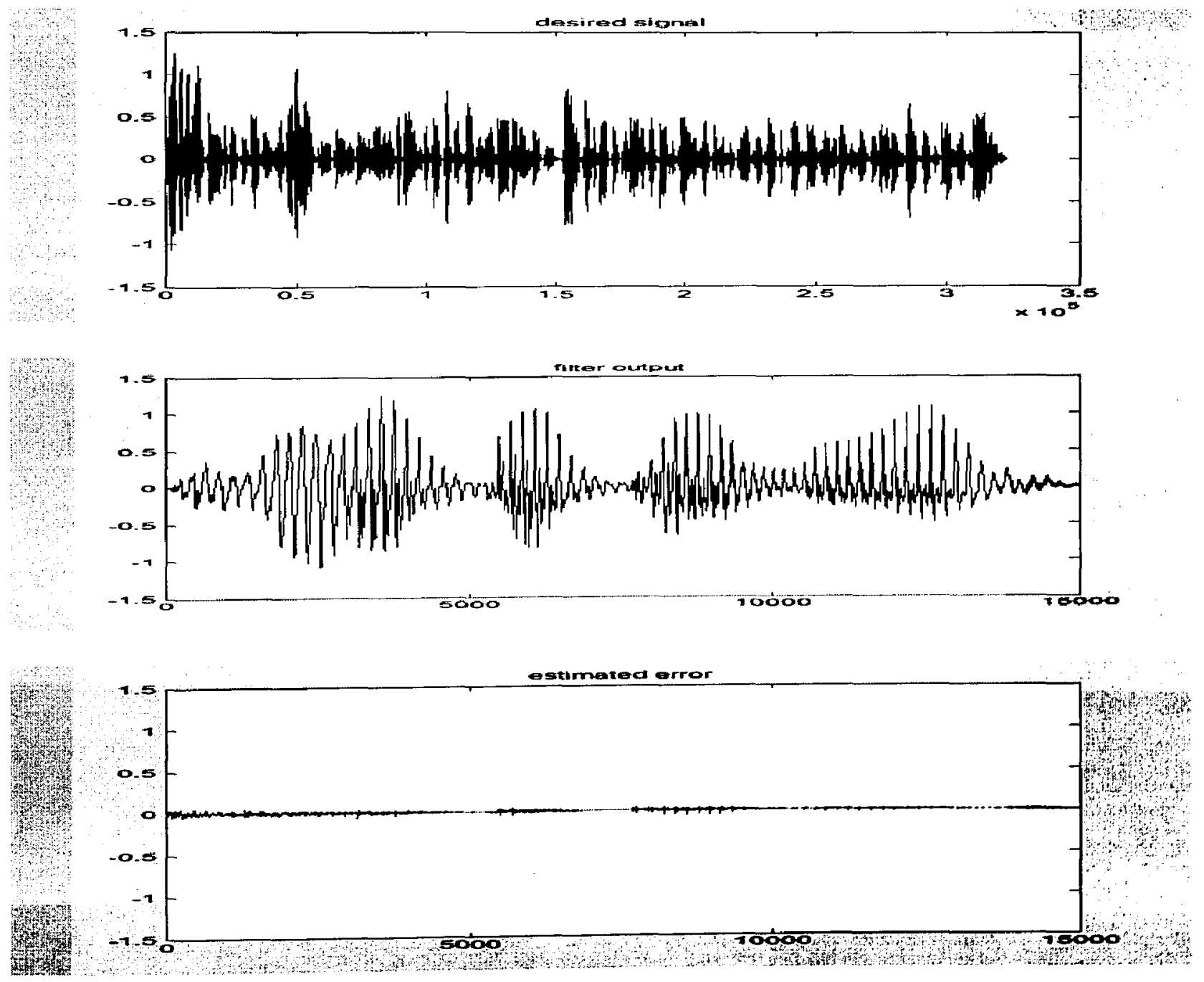


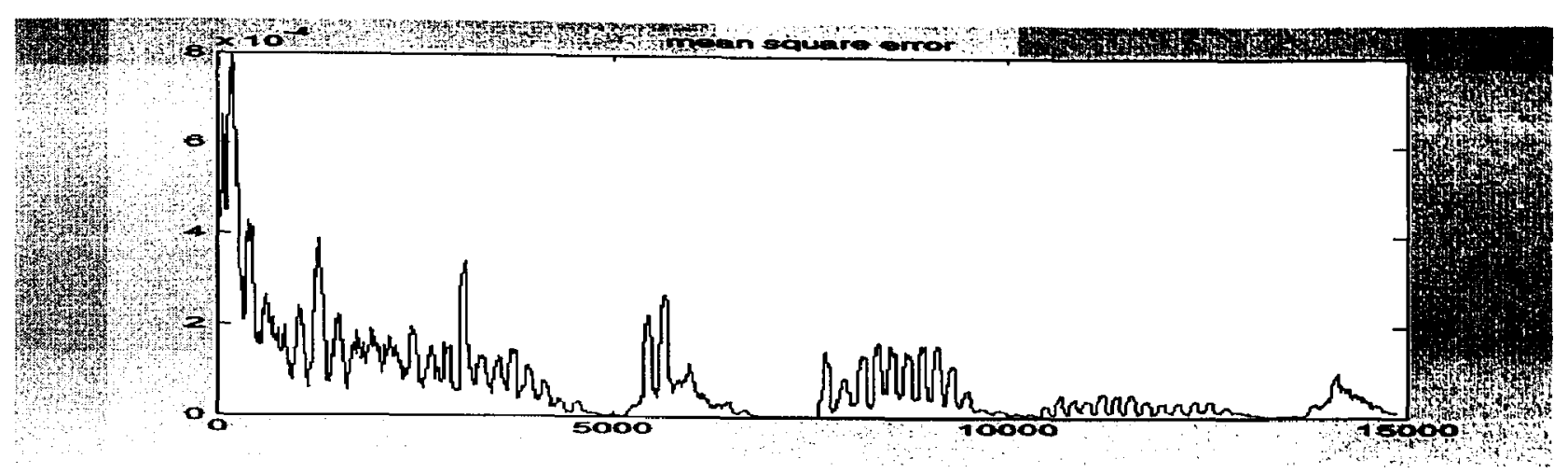

Figure 4.11 RLS algorithm
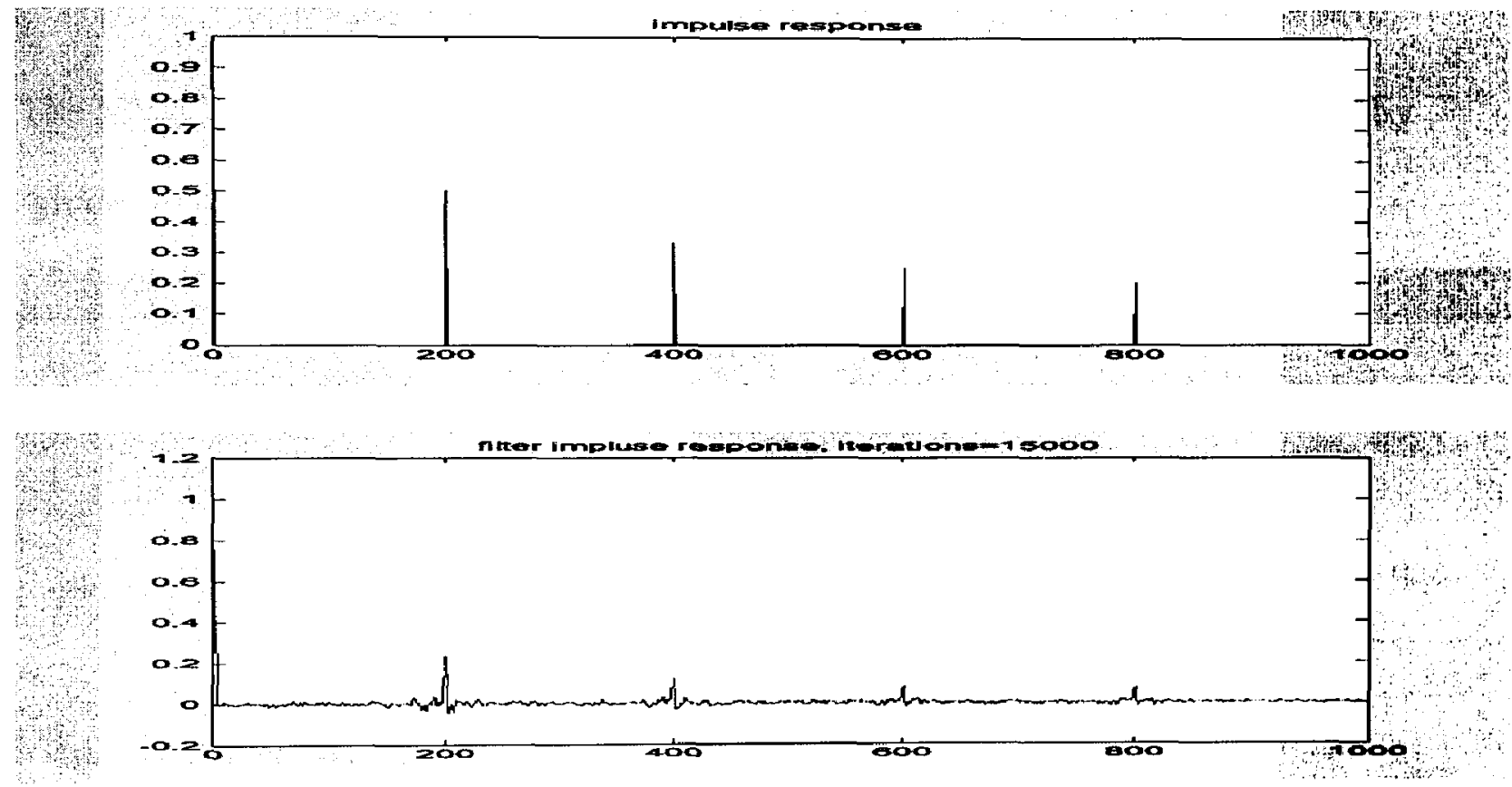

Figure 4.12 RLS FIR filter impulse response
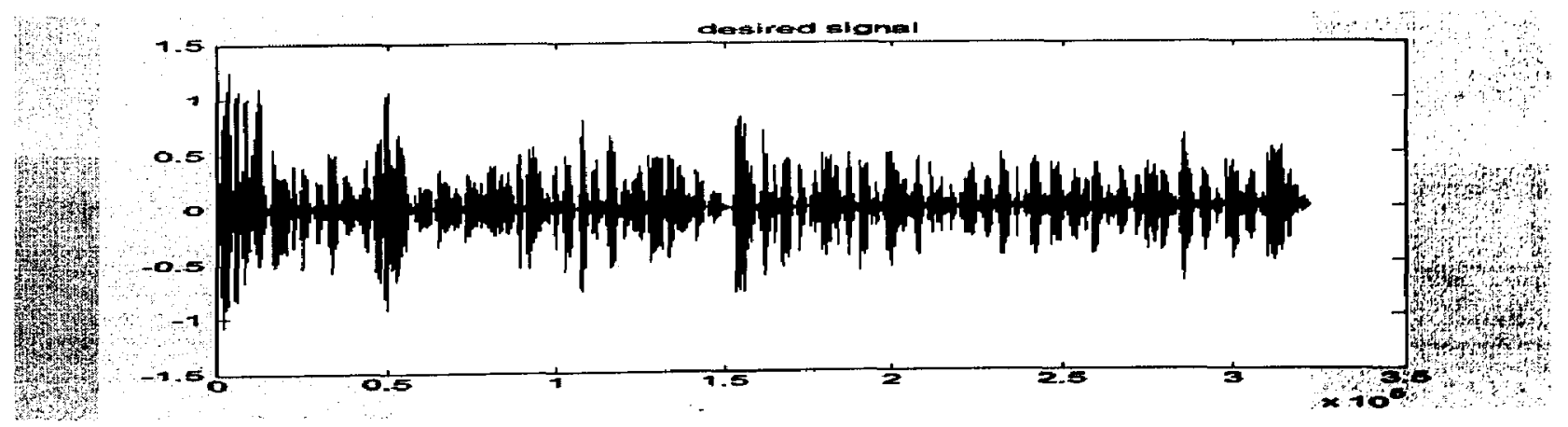

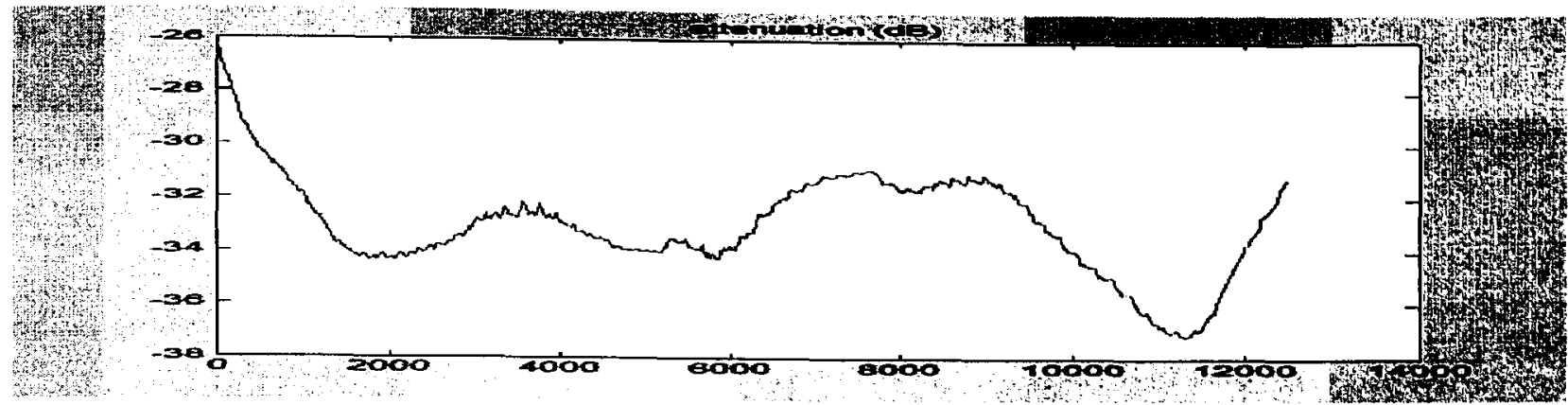

Figure 4.13 RLS echo signal attenuation

The average attenuation for RLS algorithm is $-33 \mathrm{~dB}$.

\subsection{NLMS and DTD Algorithms}

The NLMS \& DTD Analysis and Design program is another GUI program made by 'guide' to study NLMS and DTD performance, and shown in Figure 4.14.

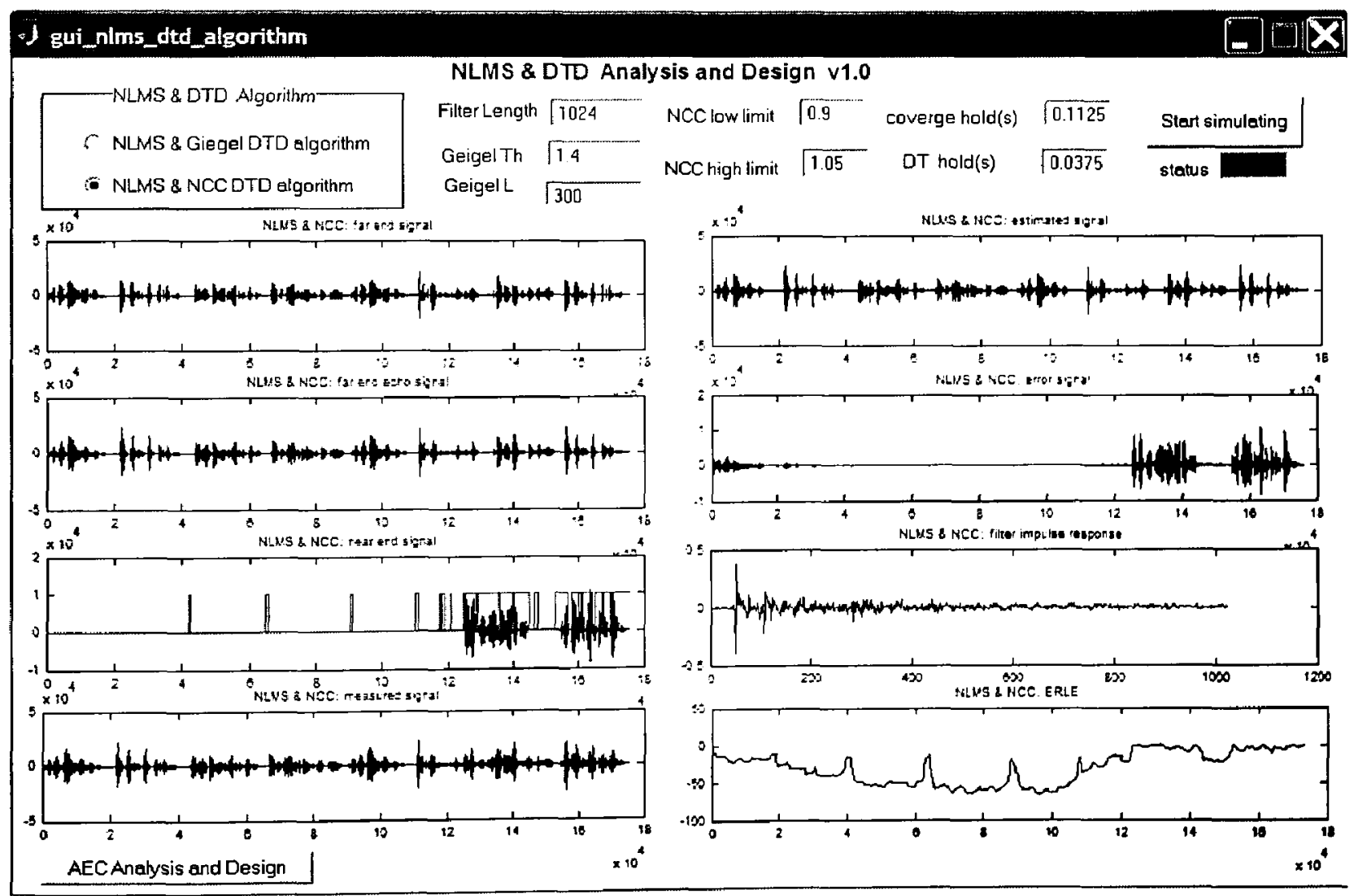

Figure 4.14 NLMS \& DTD Analysis and Design program GUI 
In this program, user can choose:

1. NLMS \& DTD Algorithm

a. NLMS and Giegel DTD algorithm

b. NLMS and normalized cross-correlation (NCC) DTD algorithm

2. Filter Length

3. Geigel Threshold

4. Geigel Length

5. NCC low limit

6. NCC high limit

7. Converge hold time

8. Double Talk period hold time

\subsubsection{The Geigel DTD algorithm}

The Figure 4.15 shows the far end signal, far end echo signal, near end signal with DTD detection, received signal, error signal, filter impulse response, and ERLE for NLMS \& Geigel DTD algorithm.
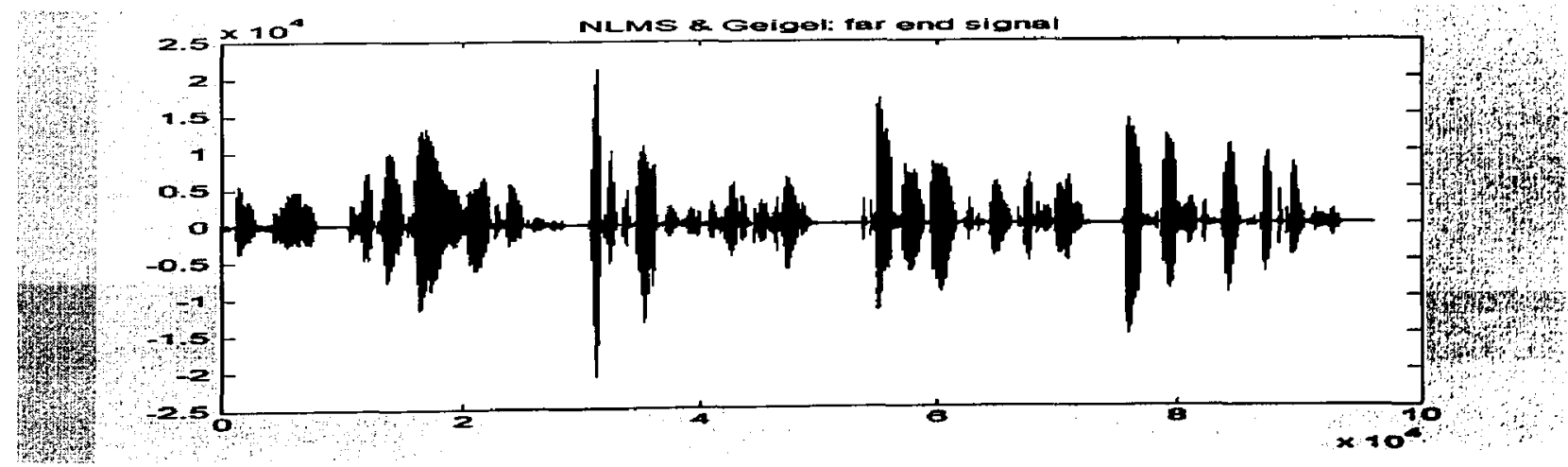

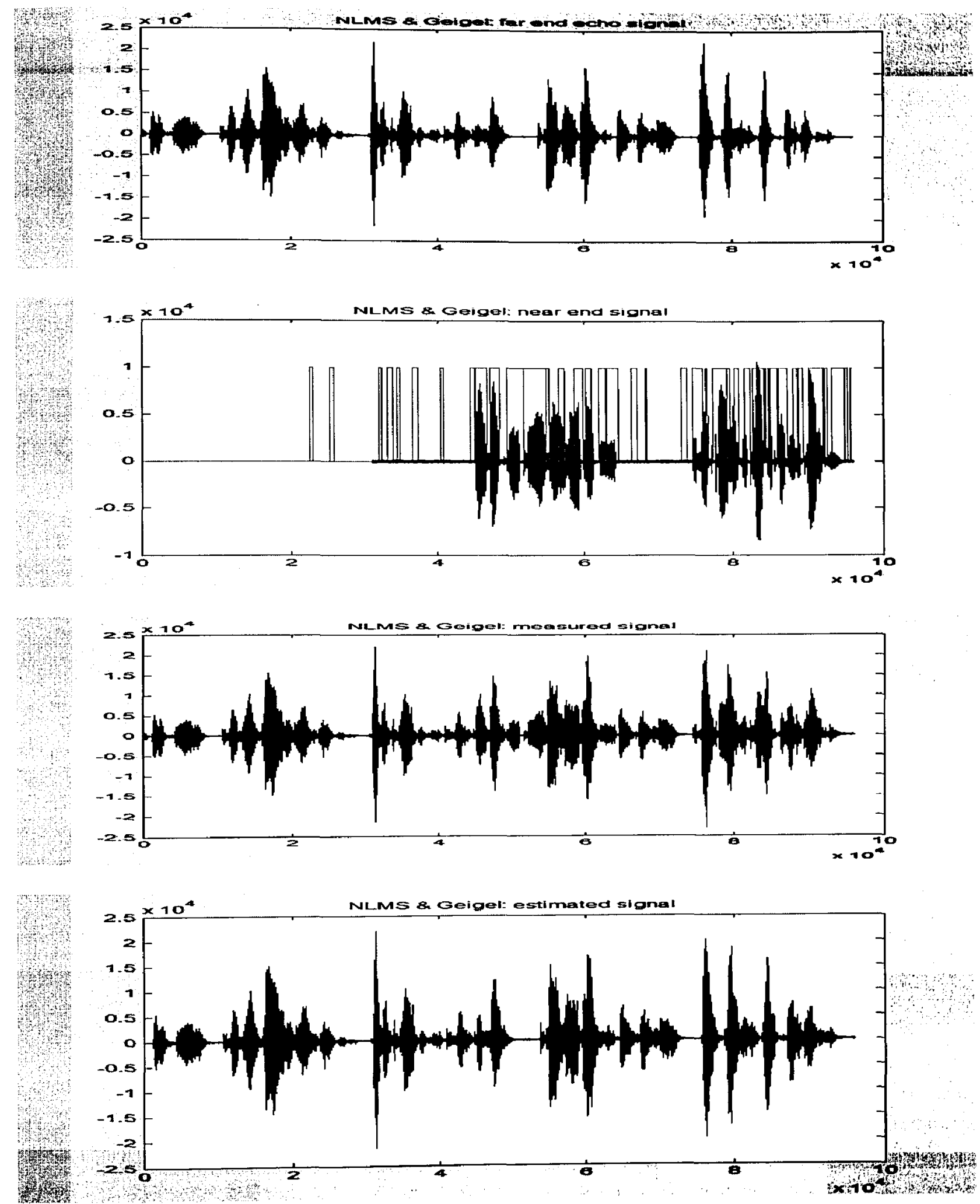

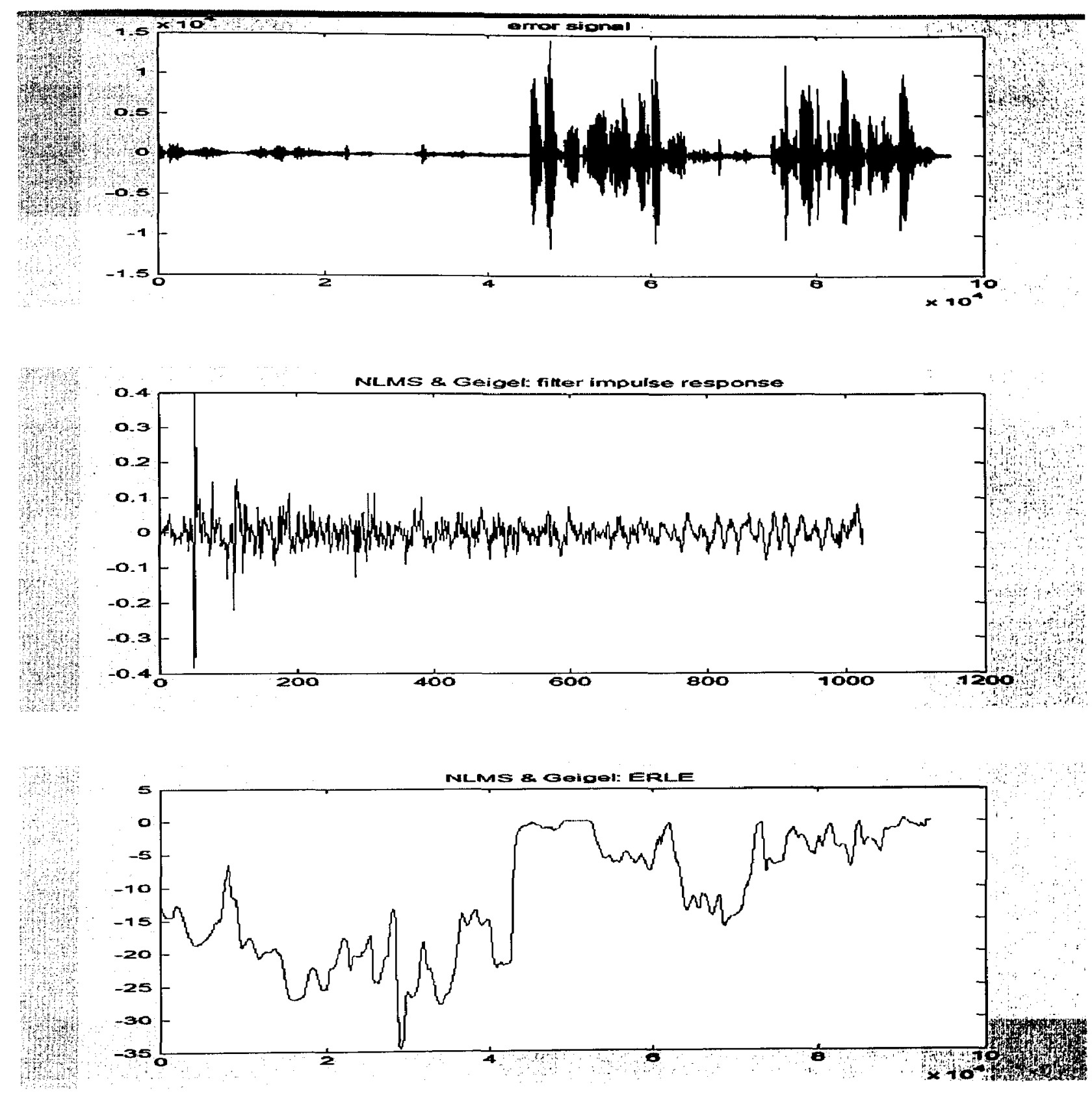

Figure 4.15 NLMS \& Geigel DTD algorithm

The Geigel DTD miss alarm rate is $P m=1-21715 / 33368=35 \%$. and its false alarm rate is $P f=15559 / 96009=16 \%$. 


\subsubsection{NLMS and normalized cross-correlation DTD algorithm}

The Figure 4.16 shows the far end signal, far end echo signal, near end signal with DTD detection, received signal, error signal, filter impulse response, and ERLE for . NLMS and The normalized cross-correlation DTD algorithm.
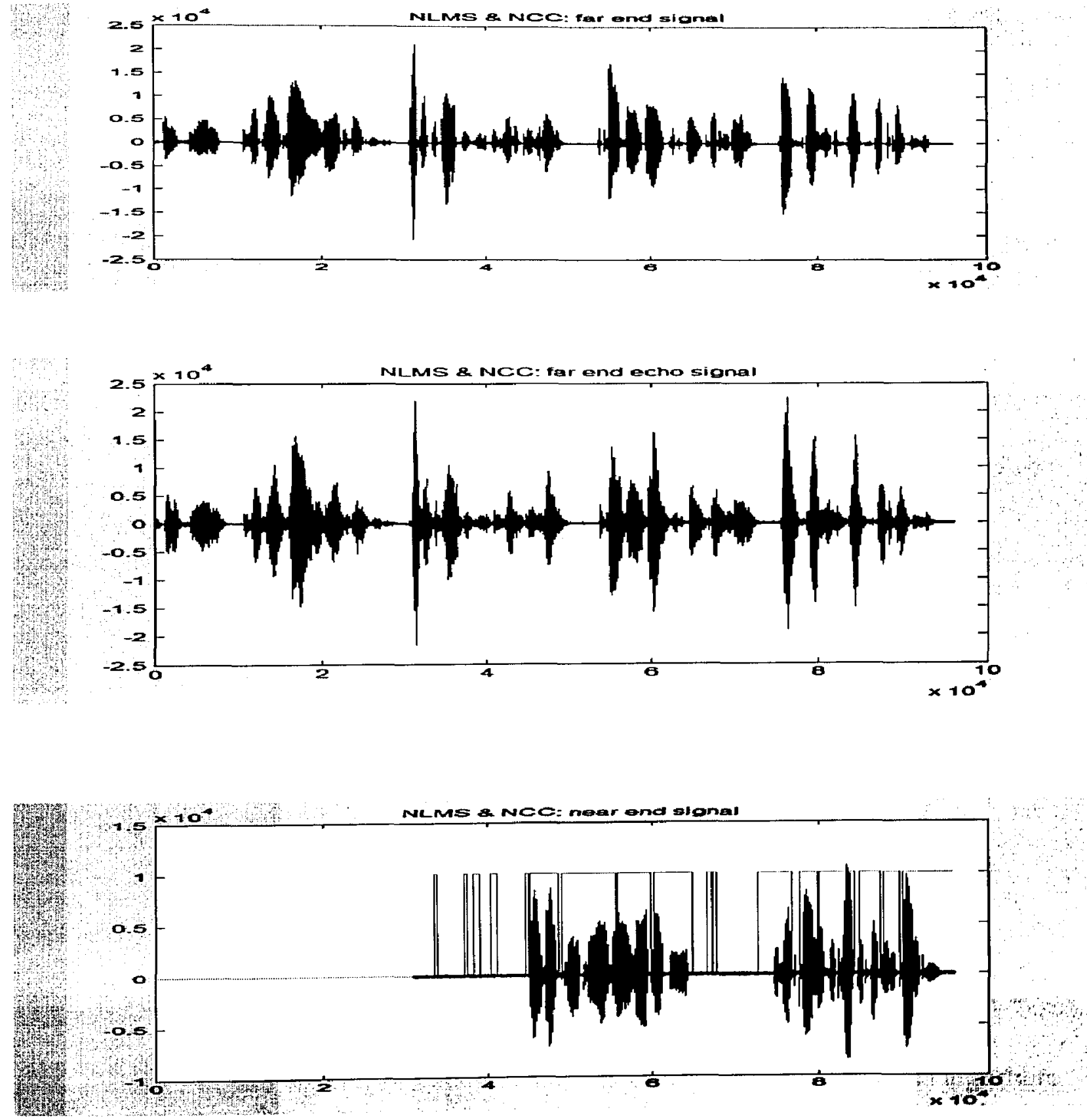

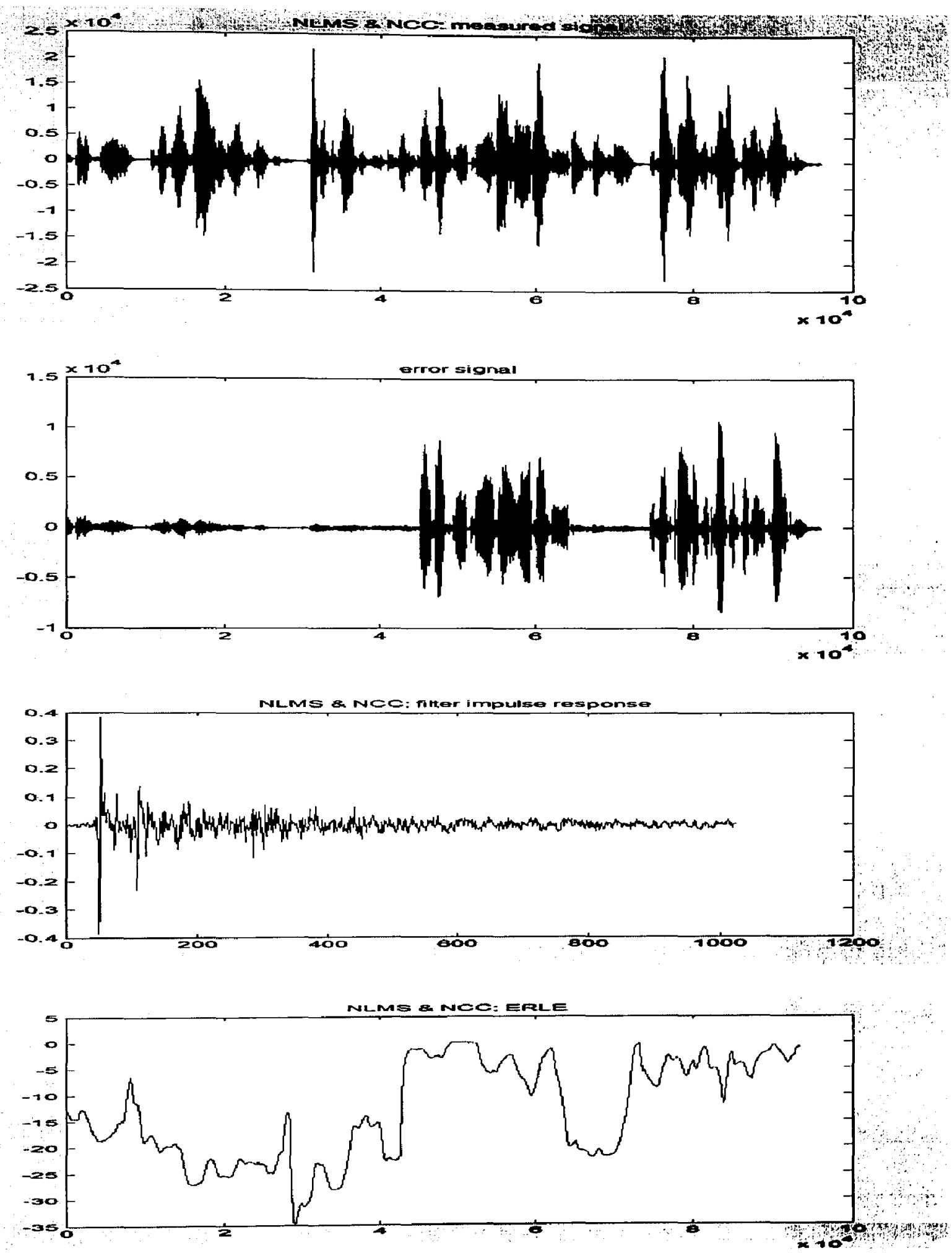

Figure 4.16 NLMS and the normalized cross-correlation DTD algorithm

The miss alarm rate is $P m=1-30118 / 33368=0.1=10 \%$ and the false alarm rate is $P f=$ $2432 / 96009=2.5 \%$. 


\subsection{Matlab Simulation Summary}

The ERLE (Echo Return Loss Enhancement) is used o assess the quality of an echo cancellation filter. ERLE, a function of the discrete-time index $n$, is defined as the ratio of the instantaneous power of signal $\mathrm{d}(\mathrm{n})$ and the instantaneous power of the residual echo $\mathrm{e}(\mathrm{n})$ :

$\operatorname{ERLE}(n)=10 * \log _{10}^{\left(P_{d}(n) / P_{e}(n)\right)}$

\begin{tabular}{|l|c|c|}
\hline Algorithm & Average ERLE & Multiplication (Filter Length: N) \\
\hline LMS & $-18.16 \mathrm{~dB}$ & $2 \mathrm{~N}+1$ \\
\hline NLMS & $-27.99 \mathrm{~dB}$ & $3 \mathrm{~N}+1$ \\
\hline Fast LMS & $-5.3 \mathrm{~dB}$ & $10 \operatorname{Nog}_{2} \mathrm{~N}+26 \mathrm{~N}$ \\
\hline RLS & $-33 \mathrm{~dB}$ & $4 \mathrm{~N}^{\wedge} 2$ \\
\hline
\end{tabular}

Table 4.1 Algorithm summary

The LMS algorithm belongs to the class of stochastic gradient algorithms and it's the simplest one. The mean square error of LMS in Figure 4.2 shows that the LMS filter's impulse response converges to the actual impulse response as the average value of the cost function decreases so that the filter could more accurately emulate the desired signal and more effectively cancel the echo signal. LMS is easily to be implemented and if the step size is correctly selected, it is stable. In Figure 4.5, the error signal and mean square error of the Normalized Least Mean Square (NLMS) algorithm is obviously smaller than those of LMS and has faster convergence speed than that of the LMS algorithm. The NLMS differs from the standard LMS algorithm in the sense that it varies the step size according to the power level of the far-end signal. Thus the convergence speed is independent of the input signal power. The average echo signal attenuation of NLMS 
algorithm is $-27.99 \mathrm{~dB}$ and is much better than the LMS average echo signal attenuation $-18.16 \mathrm{~dB}$. Due to the presence of feedback in the NLMS algorithm, there exists a possibility of it becoming unstable. The stability of the algorithm depends on the step seize parameter $\beta$. For the NLMS algorithm, the step size $\beta$ should satisfy: $0<\beta<2$ and the fastest convergence occurs when $\beta=1$. Since NLMS algorithm is easier to be implemented and the computation is not very intensive, as well as good echo cancellation performance, it is widely used in real time adaptive echo cancellation.

Figure 4.8 shows the Fast LMS error estimation signal is larger, the average echo signal attenuation is only $-5.3 \mathrm{~dB}$, but its converge speed is faster and computation complexity is lighter. The RLS algorithm is more effective than all other algorithms. Figure 4.11 shows its error estimation signal is also very small and its mean square error quickly approach to zero and its average echo signal attenuation is $-33 \mathrm{~dB}$, but each iteration of the RLS algorithm requires $4 \mathrm{~N}^{\wedge} 2$ multiplication operations, it is more intensive computation so that in practice it is not popular to be implemented.

From Figure 4.11 and Figure 4.2, we can see that RLS algorithm has a much better convergence rate that the LMS algorithm, but it comes out with more computational complexity. In addition, echo cancellation generally requires large FIR order and thus the RLS algorithm is not suitable for real time implementation.

Figure 4.15 shows the simulation results of the NLMS FIR filter combined with The Geigel DTD algorithm. When the near-end speaker is silent, the FIR has a good performance to cancel the echo signal and keep the error signal in very low level. When both the near-end and the far-end are active - the Double Talk is occurring, the error signal not only contains the echo estimation error, but also the near-end signal. 
At this time, the Geigel DTD algorithm will detect the DT period and not update the filter coefficients to prevent the echo canceller from being disturbed by the near end speaker's signal. From the miss alarm rate and false alarm rate, the Gegel algorithm is not very accurate to detect double talk.

For cross-correlation double talk detection algorithm, at the beginning, the decision

variable is simplified as $d(t)=\frac{r_{x z}^{T}(t) h}{\sigma_{z}^{2}(t)}=\frac{\tilde{\sigma}_{z}^{2}(t)}{\hat{\sigma}_{z}^{2}(t)}$

where $\quad \hat{\sigma}_{z}^{2}(t)=\hat{\sigma}_{z}^{2}(t-1)+z^{2}(t)-z^{2}(t-L)$

$$
\tilde{\sigma}_{z}^{2}(t)=\tilde{\sigma}_{z}^{2}(t-1)+z(t) \hat{y}(t)-z(t-L) \hat{y}(t-L)
$$

When double talk is presented, Figure 5.1 shows the filter output error signal is not correct and Figure 5.2 displays the double talk detection is not accurate. Through introducing the smoothed estimate and adjusting the smooth factor that described in 3.2, the better results can be achieved.

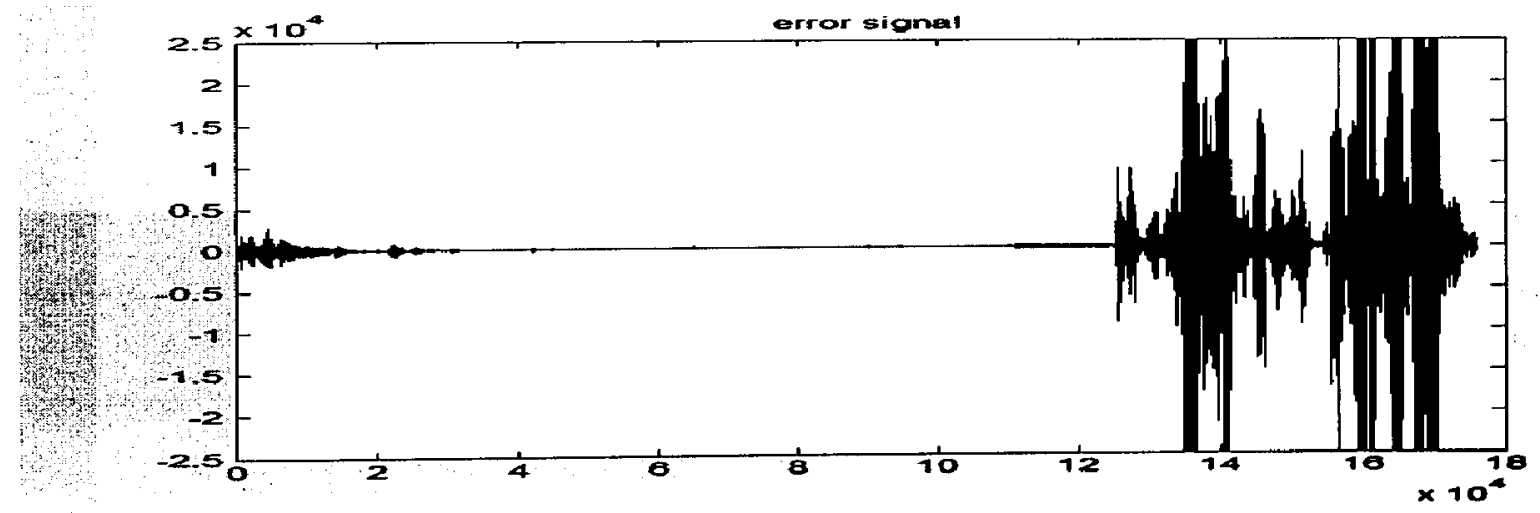

Figure 4.17 Error signal 


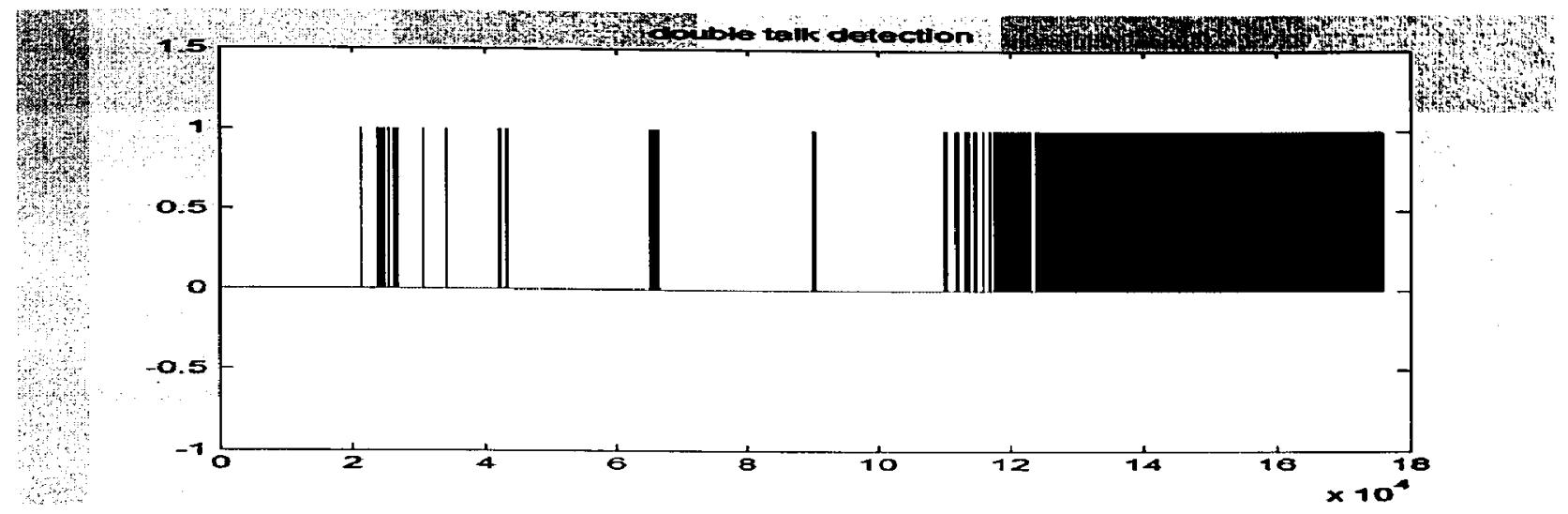

Figure 4.18 Double talk detection

Figure 4.16 shows the simulation results of the NLMS FIR filter combined with the selected normalized cross-correlation DTD algorithm described in 3.2. In no Double Talk period, the filter echo cancellation keep the same as the Geigel DTD algorithm. Figure 4.16 shows the normalized cross-correlation DTD has a better decision in distinguishing DT period. Though it causes more intensive computation, the miss alarm rate is $10 \%$ and the false alarm rate $2.5 \%$ is much better than Geigel algorithm. 


\section{Chapter 5}

\section{Adaptive Echo Canceller with DTD Structure}

\subsection{Adaptive Echo Canceller with DTD}

Based on above adaptive algorithm analysis and DTD studies, the NLMS algorithm and normalized cross-correlation DTD algorithm are finally selected in designing the AEC as shown in Figure 6.1.

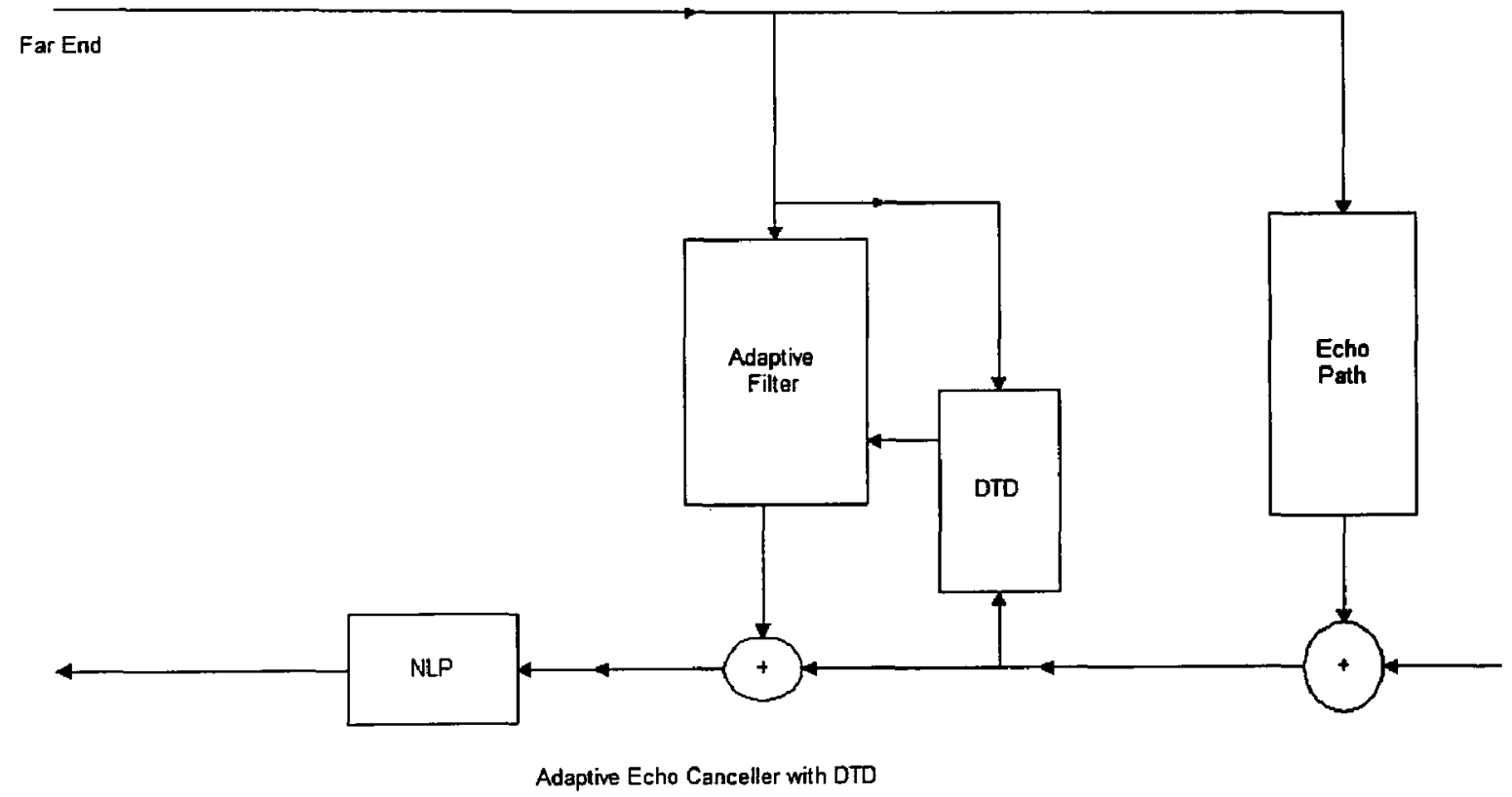

Figure 5.1 Adaptive echo canceller with DTD

The adaptive filter is based on the normalized least mean square (NLMS) algorithm and in the NLMS coefficient update equation (2.2.2),

$$
w(m)=w(m-1)+\mu \frac{e(m)}{x(m)_{A}^{T} x_{A}(m)} x_{A}(m),
$$

the variable $\mu$ is the step size, which is usually between $(0,2)$ to maintain the system stable. 
The length of the adaptive filter depends on the reverberation time constant of the room. The system is working with sample rate of $8 \mathrm{KHZ}$ and the predicted echo path is about $128 \mathrm{msec}$. Thus adaptive filter length will be set as:

Filter length $=$ echo path $*$ sample rate $/ 1000=1024$.

With the equations (3.2.7), (3.2.8) and (3.2.9) of the normalized cross-correlation DTD algorithm, the smoothing factor $\alpha$ is set to be 0.9 in the equations (3.2.8) and (3.2.9) from experiments. Thus the received decision variable $d$ is a good estimate of the theory value only after the adaptive filter converge, as well as $r_{x z}, \sigma_{z}$ become good statistical estimates. Hence the adaptive filter has to converge first to make this DTD algorithm effective. However, it won't cause much problem; because the adaptive algorithm takes relative short time to converge and remain converge most of the time. Initially, the DTD has to be switched off until the adaptive filter first time converges. To detect the convergence of the adaptive filter, we still use the same decision variable $d$. We know that the decision variable $d$ is about 1 only when the adaptive has converged and there is no double-talk. Hence, the convergence is claimed when the decision variable $d$ approaches 1 and remaining approaching 1 for a certain time. The range of approaching 1 is set to be $(0.9,1.05)$ and the certain time is set to be 0.125 second in practice. In this project, the AEC takes about 25472 samples to converge and the convergence time is calculated as:

\section{Initial Convergence Time $=25472 / 8000=3.2 \mathrm{sec}$.}

Once the convergence is claimed, we switch on the DTD. After that DTD monitors the decision variable $d$ and it claims double-talk occurs when the decision variable $d$ is out of the range $(0.75,1.35)$. Once double talk is declared, the detection is held for a minimum 
period of time. While the detection is held, the filter adaptation and non-linear processor is frozen. After the hold time, the DTD resumes monitoring again. The hold time is necessary due to the noisy behavior of the detection statistic and is set to $38 \mathrm{~ms}$ in implementation.

\subsection{Non-linear processor}

Since the residual echo is inevitable for the non-linearity of the echo path, the non-linear processor (NLP) is used to degrade the residual echo to an inaudible level. Only during single talk, NLP is active and it is controlled by the DTD. The NLP in this project is set as a controlled attenuator, which attenuates the echo during single-talk by $20 \mathrm{~dB}$. 


\subsection{Software Workflow}

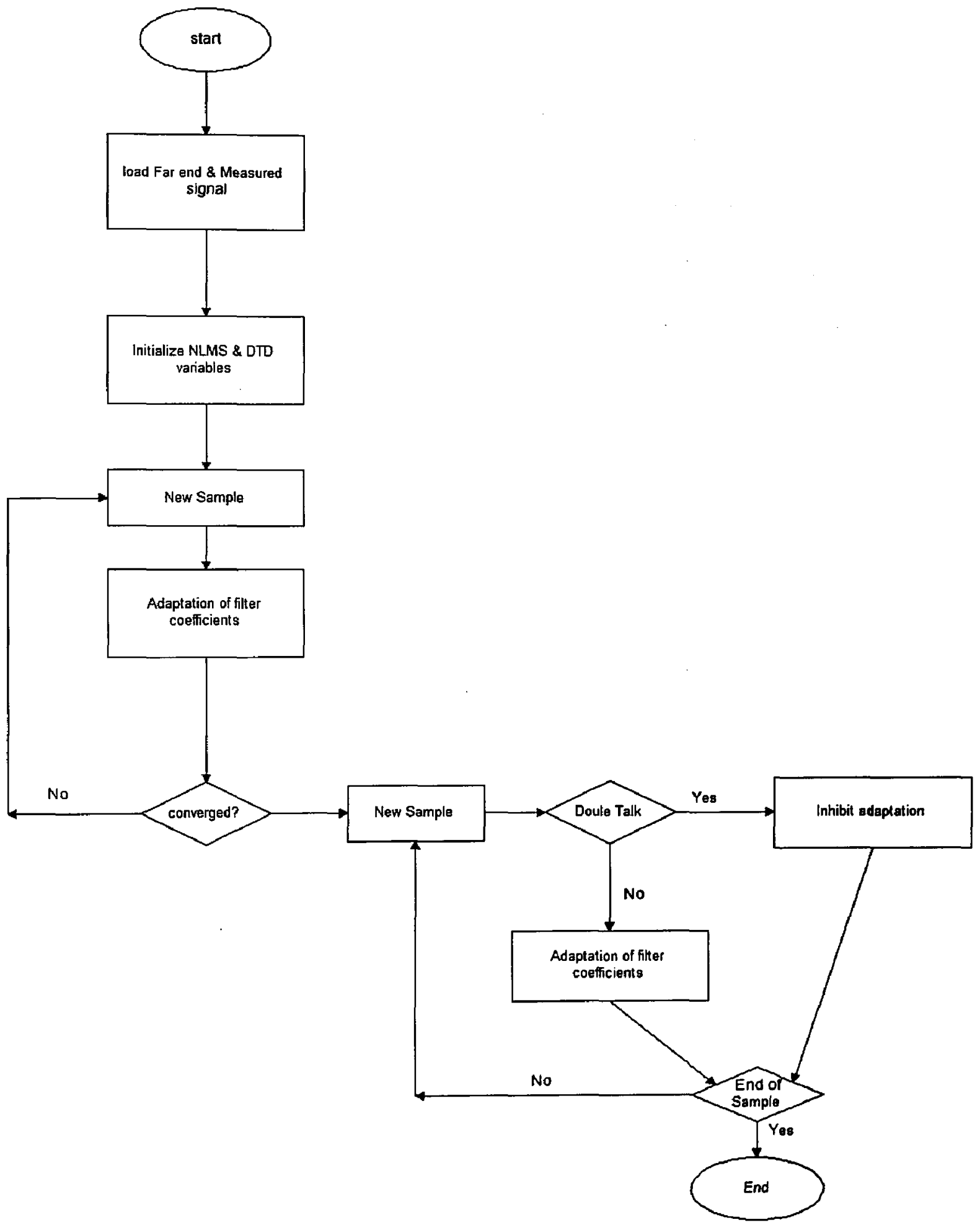

Figure 5.2 Adaptive echo canceller with DTD software workflow 


\section{Chapter 6}

\section{ITU G.168 Test Cases}

The International Telecommunications Union (ITU), as well as the European

Telecommunication Standard Institute (ETSI) regulated the specifications of telephone systems. The ITU-T recommendations: G.167 specifies the performance requirements of acoustic echo control devices. G.165 specifies the requirements of network echo canceller. G.168 is an enhanced version and specifies the new requirements of digital network echo canceller.

\subsection{Test 1: Steady state residual and residual echo level test}

The G.168 Requirements for Test 1: In the Table 6.1 below, "L(Rin) Input", are the various input levels. "L(Res), NLP Dis, Output Req." are the requirements per G.168 (2000). "Sample" column is for the users to record their test results.

\begin{tabular}{|l|l|l|l|l|}
\hline $\begin{array}{l}\text { LRin) } \\
\text { Input }\end{array}$ & $\begin{array}{l}\text { L(Res), NLP Dis } \\
\text { Output Req. }\end{array}$ & Result & $\begin{array}{l}\text { L(Res), NLP enab } \\
\text { Output Req. }\end{array}$ & $\begin{array}{l}\text { Result } \\
\text { (NLP Enabled) }\end{array}$ \\
\hline$-10 \mathrm{dBm} 0$ & $-37 \mathrm{dBm} 0$ & $-46.15 \mathrm{dBm} 0$ & $-65 \mathrm{dBm} 0$ & $-66.15 \mathrm{dBm} 0$ \\
\hline
\end{tabular}

Table 6.1 Test 1 results

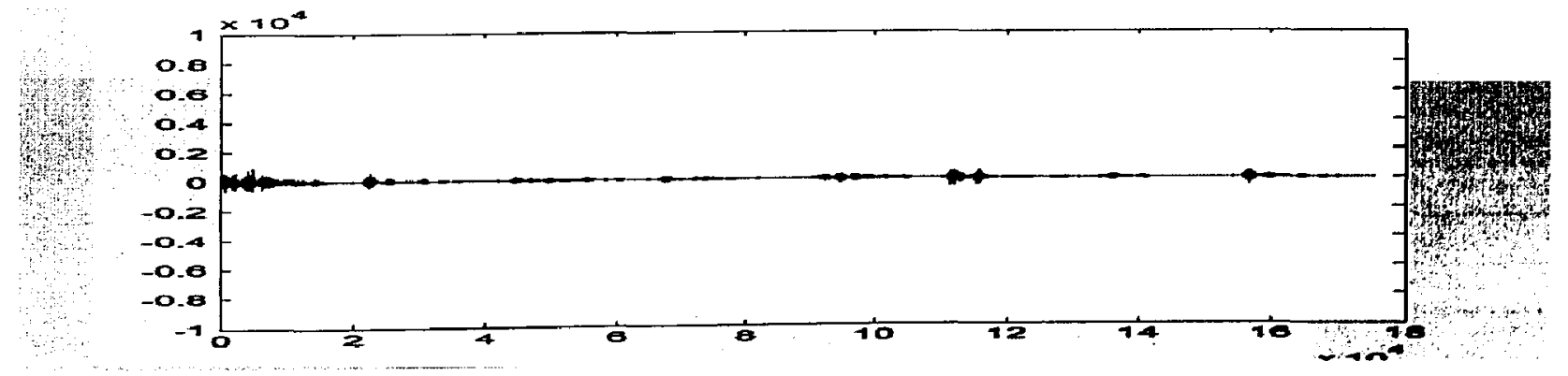

error signal

Figure 6.1 Test 1 


\subsection{Test 2A and 2B: Convergence test with NLP enabled and disabled}

\subsubsection{Test 2A: Convergence test with NLP enabled}

The G.168 Requirements for Test 2A: the echo path delay is considered as time "zero".

For $50 \mathrm{~ms}$ beyond the echo path delay, the $\mathrm{EC}$ is required to do no better than the

minimum ERL of $6 \mathrm{~dB}$. Between $50 \mathrm{~ms}$ and $1 \mathrm{sec}$, the performance of the EC must

increase from the minimum of $6 \mathrm{~dB}$ to up to $20 \mathrm{~dB}$. Beyond $1 \mathrm{sec}$, the EC performance is dependent on the input signal level and should be $55 \mathrm{dBm} 0$ or greater for input signal levels of $0 \mathrm{dBm} 0$ and $-10 \mathrm{dBm} 0$.

\begin{tabular}{|l|l|l|}
\hline Time (ms) & $\begin{array}{l}\text { L (Rin)-L (Ret) } \\
\text { (NLP Enabled) }\end{array}$ & $\begin{array}{l}\text { Result } \\
\text { (NLP Enabled) }\end{array}$ \\
\hline 0 & $6 \mathrm{~dB}$ & $48 \mathrm{~dB}$ \\
\hline $50 \mathrm{~ms}-1 \mathrm{~s}$ & $6 \mathrm{~dB} \sim 20 \mathrm{~dB}$ & $50 \mathrm{~dB}$ \\
\hline $1 \mathrm{~s}+$ & @ $-10 \mathrm{dBm} 055 \mathrm{~dB}$ & $57 \mathrm{~dB}$ \\
\hline
\end{tabular}

Table 6.2 Test $2 \mathrm{~A}$ results

\subsubsection{Test 2B: Convergence test with NLP disabled}

The G.168 Requirements for Test 2B: the echo path delay is considered time "zero".

From 0 to $50 \mathrm{~ms}$, the $\mathrm{EC}$ is not required to perform, therefore the $6 \mathrm{~dB}$ minimum requirement (equal to the minimum ERL). Between $50 \mathrm{~ms}$ to $1 \mathrm{sec}$, the $\mathrm{EC}$ is required to increase its performance to $20 \mathrm{~dB}$ of loss and maintain at least this requirement for up to $10 \mathrm{sec}$. Beyond $10 \mathrm{sec}$, the requirement is dependent on signal level.

\begin{tabular}{|l|l|l|}
\hline Time & $\begin{array}{l}\text { L (Rin)-L (Ret) } \\
\text { (NLP Disabled) }\end{array}$ & $\begin{array}{l}\text { Result } \\
\text { (NLP Disabled) }\end{array}$ \\
\hline 0 & $6 \mathrm{~dB}$ & $8.8 \mathrm{~dB}$ \\
\hline $50 \mathrm{~ms}-1 \mathrm{~s}$ & $6 \mathrm{~dB} \sim 20 \mathrm{~dB}$ & $9 \mathrm{~dB} \sim 20 \mathrm{~dB}$ \\
\hline $1 \mathrm{~s}-10 \mathrm{~s}$ & $20 \mathrm{~dB}$ & $40 \mathrm{~dB}$ \\
\hline $10 \mathrm{~s}+$ & $@-10 \mathrm{dBm} 027 \mathrm{~dB}$ & $42 \mathrm{~dB}$ \\
\hline
\end{tabular}

Table 6.3 Test $2 \mathrm{~B}$ results 


\subsection{Test $2 \mathrm{C}$ : Convergence test in the presence of background noise}

The G.168 Requirements for Test 2C Convergence Test with NLP Enabled (part (a)).

The columns are interpreted as follows. "L(Rin) Input" is the input signal level. There are three separate files provided for levels $-0 \mathrm{dBm} 0,-10 \mathrm{dBm} 0$, and $-20 \mathrm{dBm} 0$. The "Noise Level L(Rin) -15 " is the noise level mixed in along with the echo. Note that the maximum level of the noise is $-30 \mathrm{dBm} 0$. " $\mathrm{L}$ (Ret) Requirement" is the appropriate requirement from G.168 (2000) specification.

\begin{tabular}{|l|l|l|l|}
\hline $\begin{array}{l}\text { L(Rin) } \\
\text { Input }\end{array}$ & $\begin{array}{l}\text { Noise Level } \\
\text { L(Rin)-15 }\end{array}$ & $\begin{array}{l}\text { L(Ret) } \\
\text { Requirement }\end{array}$ & $\begin{array}{l}\text { Result } \\
\text { (NLP Enabled) }\end{array}$ \\
\hline$-10 \mathrm{dBm} 0$ & $-30 \mathrm{dBm} 0$ & $-30 \mathrm{dBm} 0$ & $-46 \mathrm{dBm} 0$ \\
\hline
\end{tabular}

Table 6.4 Test $2 \mathrm{C}(\mathrm{a})$ results

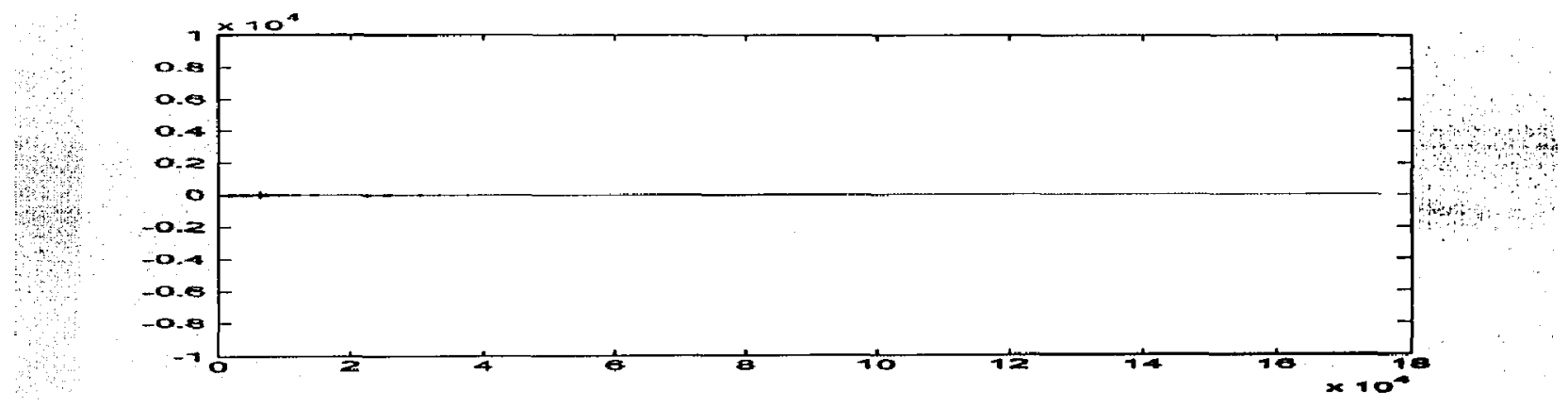

error signal

Figure 6.2 Test 2C(a)

The G.168 Requirements for Test 2C Steady State Test with NLP Enabled (part

(b)). The columns are interpreted as follows. "L(Rin) Input" is the input signal level. The

"Noise Level $-55 \mathrm{dBm0"}$ is the noise level mixed in along with the echo. "L(Ret)

Requirement" is the appropriate requirement from G.168 (2000) specification.

\begin{tabular}{|c|c|c|c|}
\hline $\begin{array}{l}\text { (Rin) } \\
\text { nput }\end{array}$ & Noise Level & $\begin{array}{l}\text { L(Ret) } \\
\text { Requirement }\end{array}$ & $\begin{array}{l}\text { Resulfy } \\
\text { (NLPEnabled) }\end{array}$ \\
\hline$-10 \mathrm{dBm} 0$ & $-55 \mathrm{dBm} 0$ & $-38 \mathrm{dBm} 0$ & $-46 \mathrm{dBm} 0$ \\
\hline
\end{tabular}

Table 6.5 Test $2 \mathrm{C}(\mathrm{b})$ results 


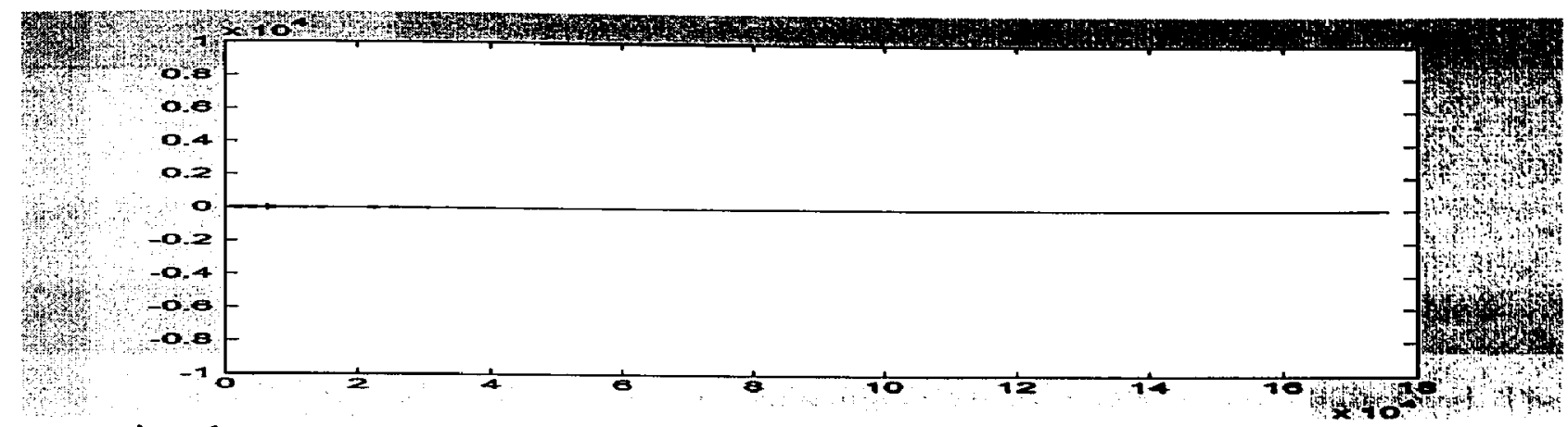

error signal

Figure 6.3 Test $2 \mathrm{C}(\mathrm{b})$

The G.168 Requirements for Test 2C with NLP disabled (part (c)). The columns are interpreted as follows. The input requirements with NLP disabled are similar to those for NLP enabled. The corresponding requirements are given in the column " $L($ Ret)

Requirement”.

\begin{tabular}{|l|l|l|l|}
\hline E(Rin) & $\begin{array}{l}\text { Noise Level } \\
\text { L(Rin)-15 }\end{array}$ & $\begin{array}{l}\text { L(Ret) } \\
\text { Requirement }\end{array}$ & $\begin{array}{l}\text { Sample } \\
\text { (NLP Disabled) }\end{array}$ \\
\hline$-10 \mathrm{dBm} 0$ & $-30 \mathrm{dBm} 0$ & $-30 \mathrm{dBm} 0$ & $-37 \mathrm{dBm} 0$ \\
\hline
\end{tabular}

Table 6.6 Test $2 \mathrm{C}(\mathrm{c})$ results

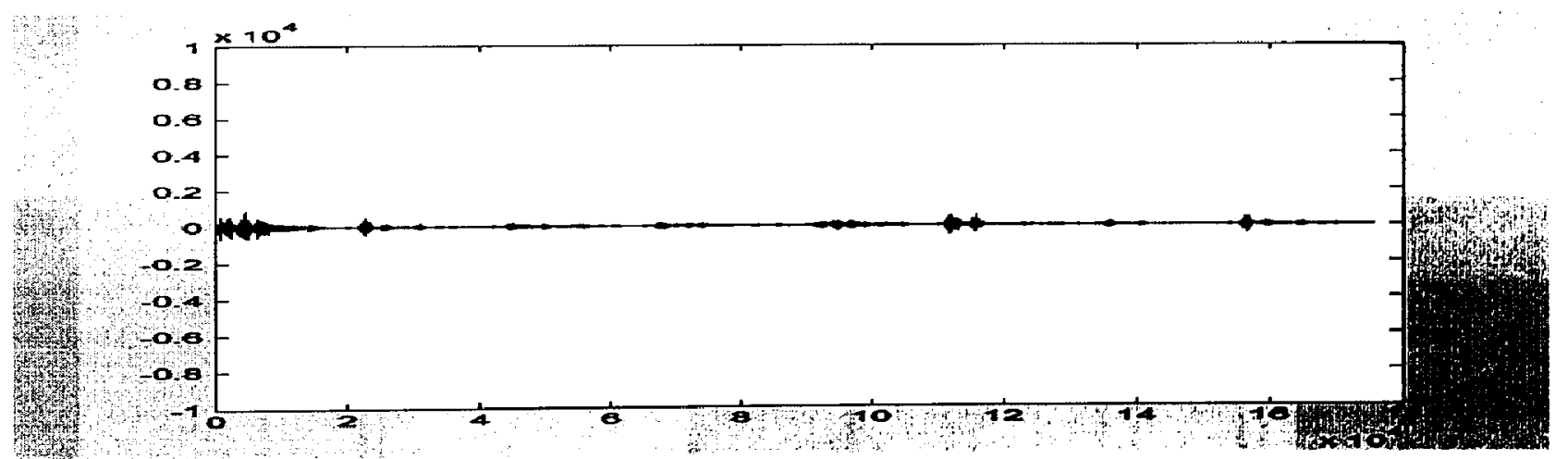

error signal

Figure 6.4 Test $2 \mathrm{C}(\mathrm{c})$ 


\subsection{Test 3: Performance under conditions of double talk}

\subsubsection{Test 3A: Double talk test with low near end levels}

The G.168 Requirements for Test 3A: the requirement as given in the Table 6.7 below is such that the residual echo level should be equal to lower than the doubletalk level. The convergence occurs during the periods when the echo and doubletalk signals do not overlap.

\begin{tabular}{|l|l|l|l|}
\hline $\begin{array}{l}\text { E(Rin) } \\
\text { Input }\end{array}$ & $\begin{array}{l}\text { Low Near-End } \\
\text { L(Rin)-15 }\end{array}$ & $\begin{array}{l}\text { Level L(Res) } \\
\text { Requirement }\end{array}$ & $\begin{array}{l}\text { Result } \\
\text { (NLP Disabled) }\end{array}$ \\
\hline$-10 \mathrm{dBm} 0$ & $-25 \mathrm{dBm} 0$ & $-25 \mathrm{dBm} 0$ & $-34 \mathrm{dBm} 0$ \\
\hline
\end{tabular}

Table 6.7 Test $3 \mathrm{~A}$ results

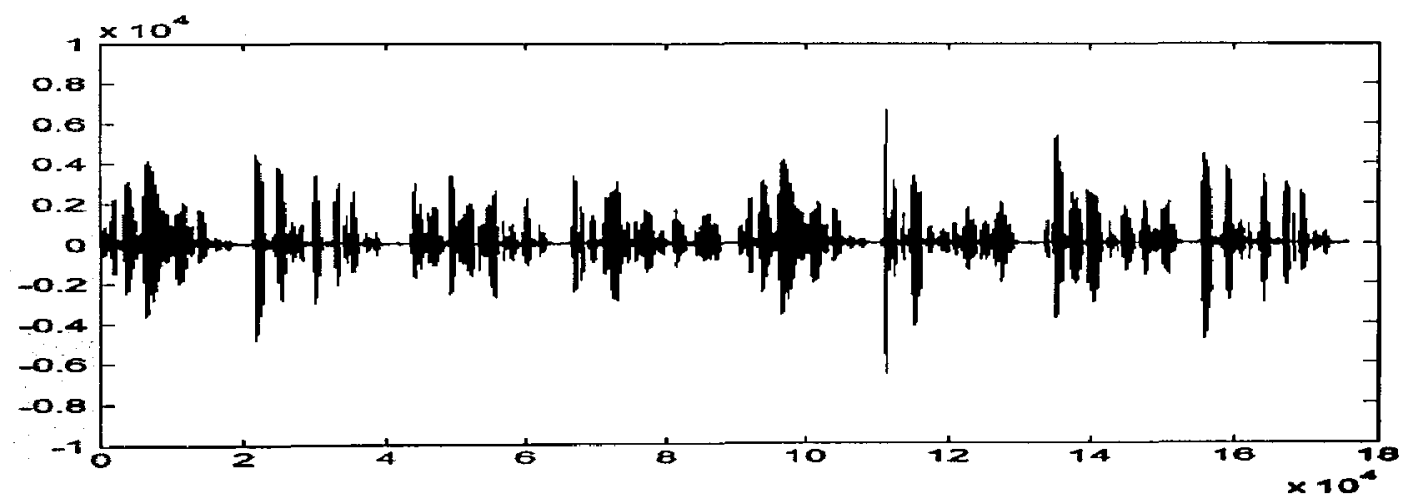

far end signal

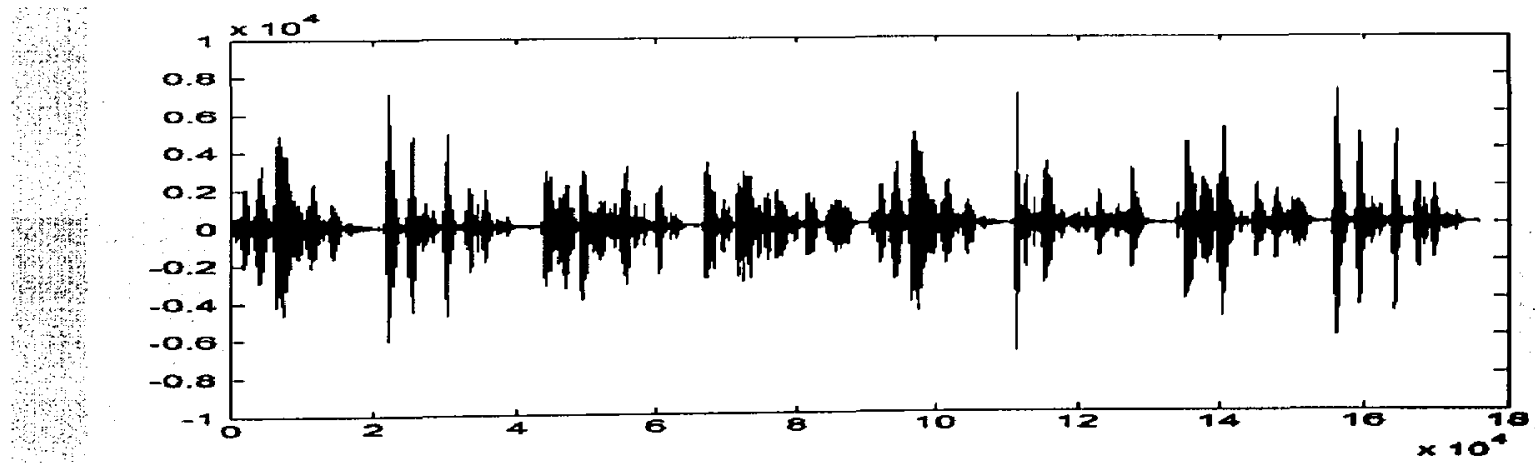

fare end echoed signal 


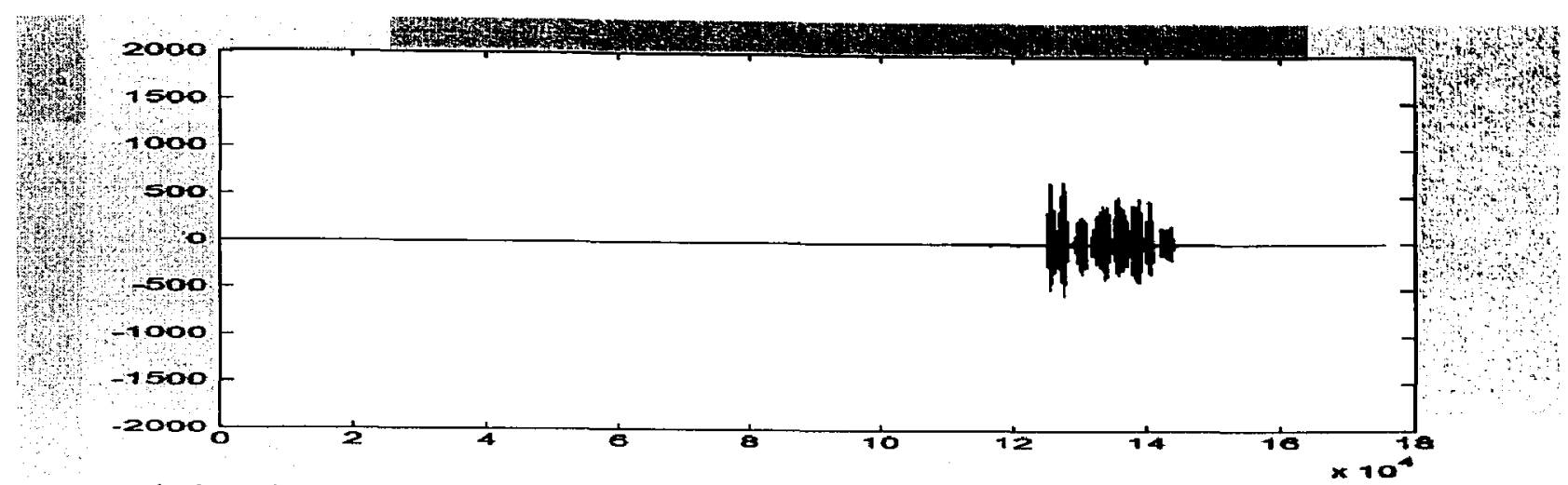

near end signal

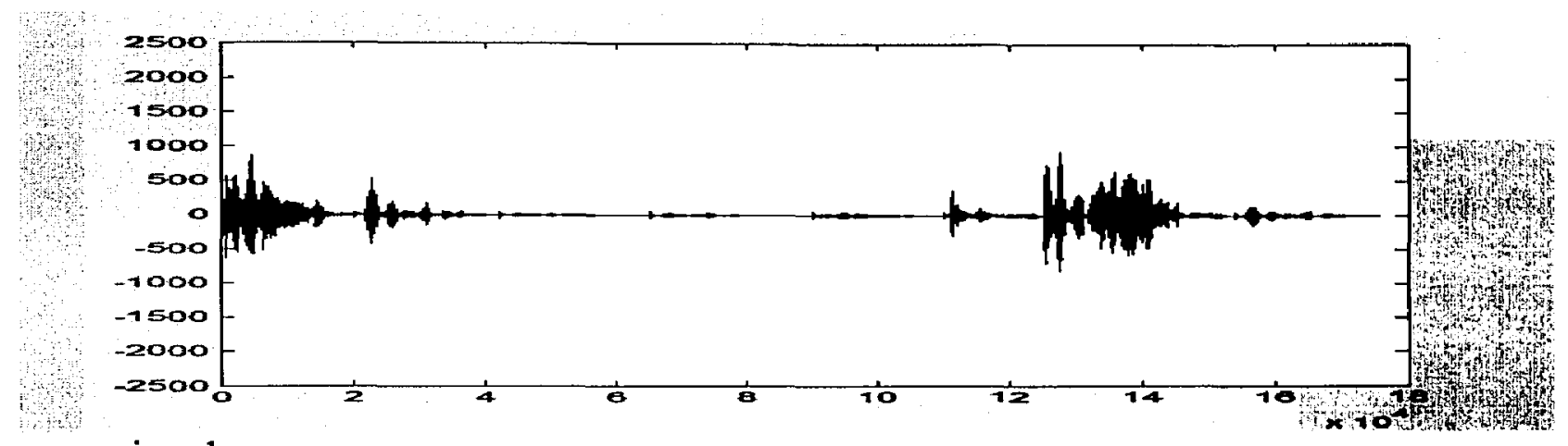

error signal

Figure 6.5 Test $3 \mathrm{~A}$

\subsubsection{Test 3B: Double talk test with high near end levels}

The G.168 Requirements for Test 3B: notice that the doubletalk level should be at least as great as the input level. The residual echo requirement is relaxed by $10 \mathrm{~dB}$ from the steady state requirements.

\begin{tabular}{|c|c|c|c|}
\hline $\begin{array}{l}\text { Wopin) } \\
\text { Input }\end{array}$ & $\begin{array}{l}\text { Doubletalk } \\
\mathbf{N} \geq \mathbf{L} \text { (Rin) }\end{array}$ & $\begin{array}{l}\text { Level L(Ret) } \\
\text { Requirement }\end{array}$ & $\begin{array}{l}\text { Resulth } \\
\text { (NLP Disabled) }\end{array}$ \\
\hline$-10 \mathrm{dBm} 0$ & $-10 \mathrm{dBm} 0$ & $-27 \mathrm{dBm} 0$ & $-35 \mathrm{dBm} 0$ \\
\hline
\end{tabular}

Table 6.8 Test $3 \mathrm{~B}$ results 

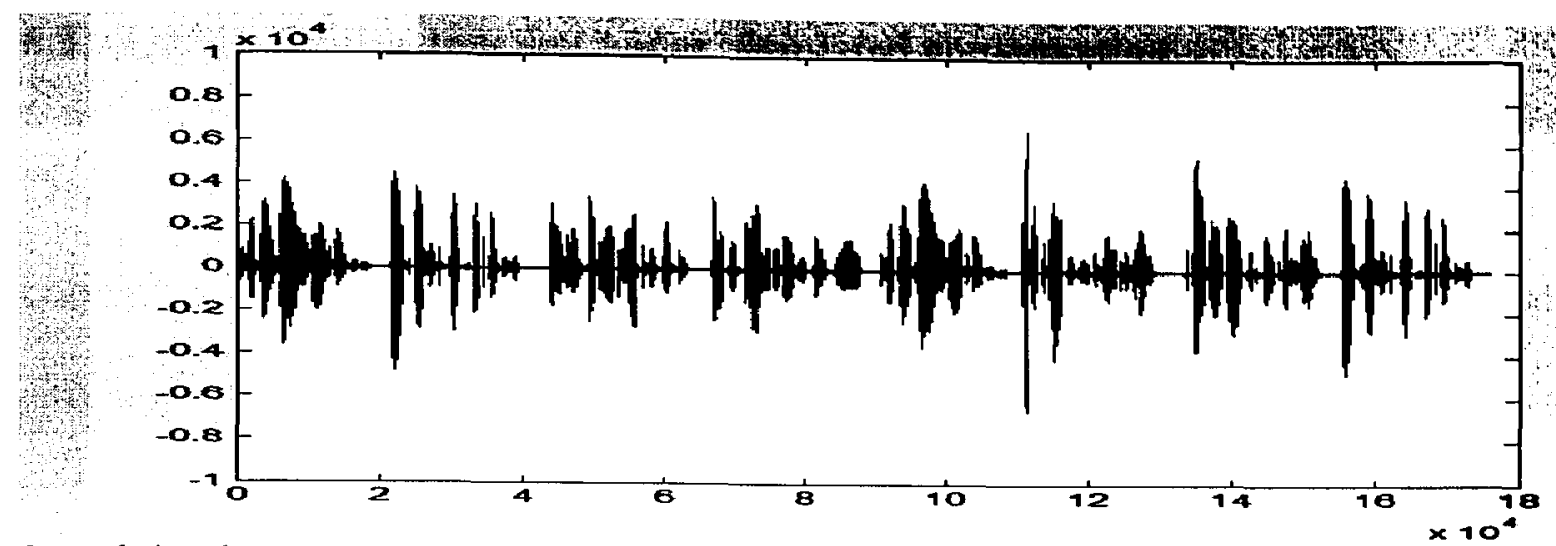

far end signal

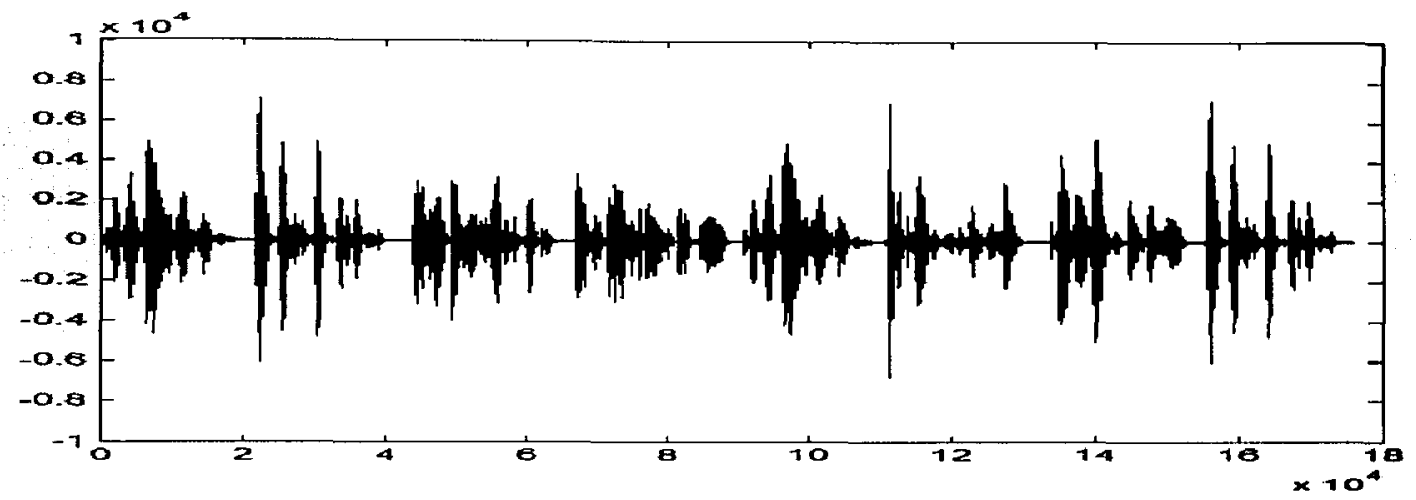

far end echoed signal

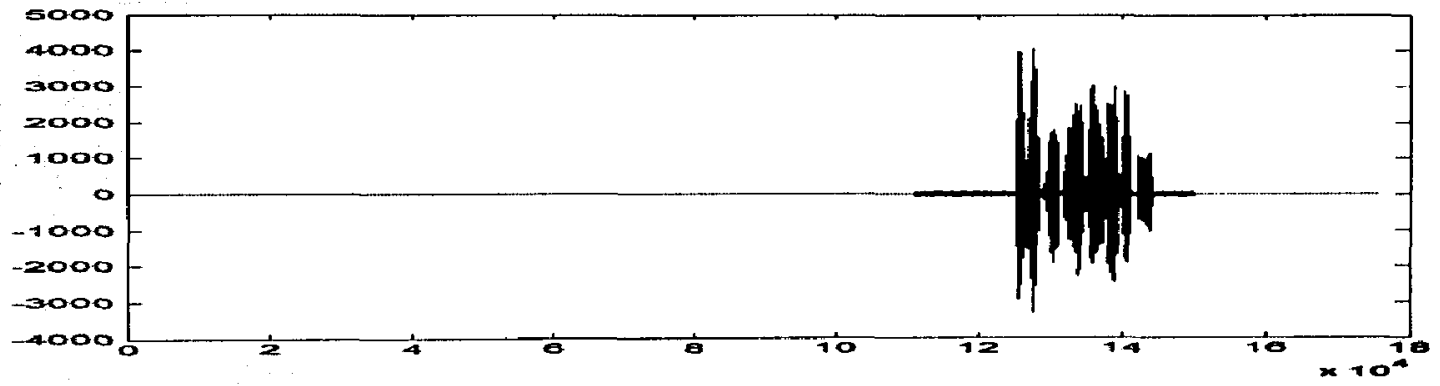

near end signal

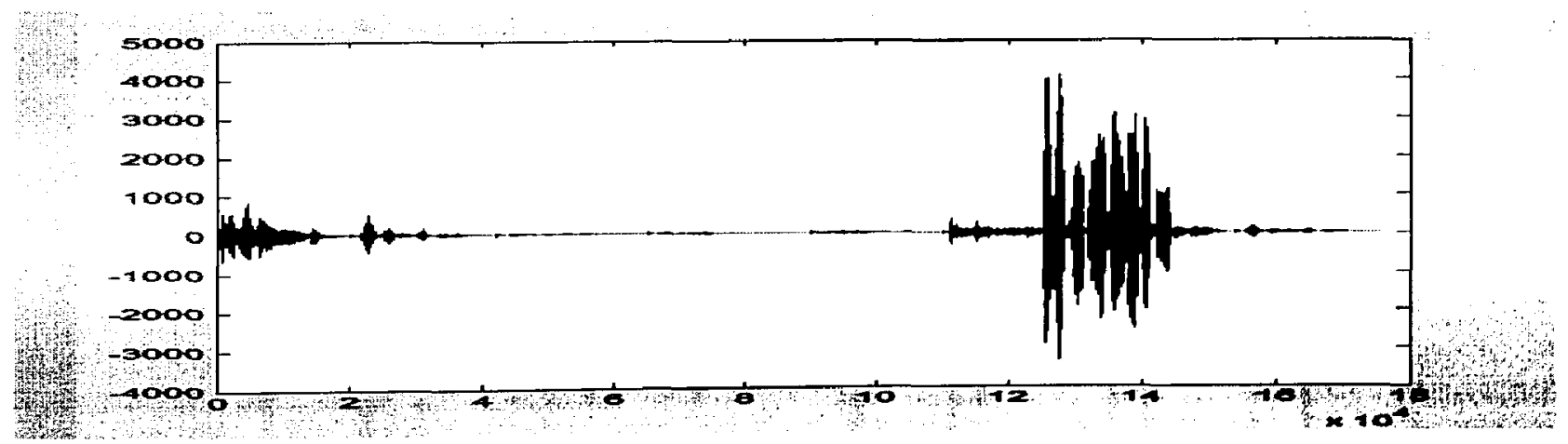

error signal

Figure 6.6 Test 3B 


\subsection{Test 3C: Double talk test under simulated conversation}

The G.168 Requirements for Test 3C: in the Table 6.9 below, note that the table continues, i.e. there are actually eight (8) columns. The doubletalk signal is applied simultaneously with the input signal. The resultant signal is divided into 5 periods (refer to G.168 (2000) spec) and denoted as $t_{1}, t_{2}, t_{3}, t_{4}$, and $t_{5}$. The requirements for these different periods are given in the table below.

\begin{tabular}{|l|l|l|l|}
\hline $\begin{array}{l}\text { L(Rin) } \\
\text { Input }\end{array}$ & $\begin{array}{l}\text { Doubletalk Level } \\
\text { N Performance }\end{array}$ & t2(Req.) & Result \\
\hline$-10 \mathrm{dBm} 0$ & $-10 \mathrm{dBm} 0$ & no peaks $>\mathrm{N}$ & meets req. \\
\hline
\end{tabular}

\begin{tabular}{|l|l|l|l|}
\hline $\begin{array}{l}\text { t3(Req.) } \\
\text { Performance }\end{array}$ & Result & t4(Req.) & Result \\
\hline$-65 \mathrm{dBm} 0$ & $-52 \mathrm{dBm} 0$ & no peaks $>\mathrm{N}+6 \mathrm{dBm} 0$ & meets req. \\
\hline
\end{tabular}

Table 6.9 Test $3 \mathrm{C}$ results

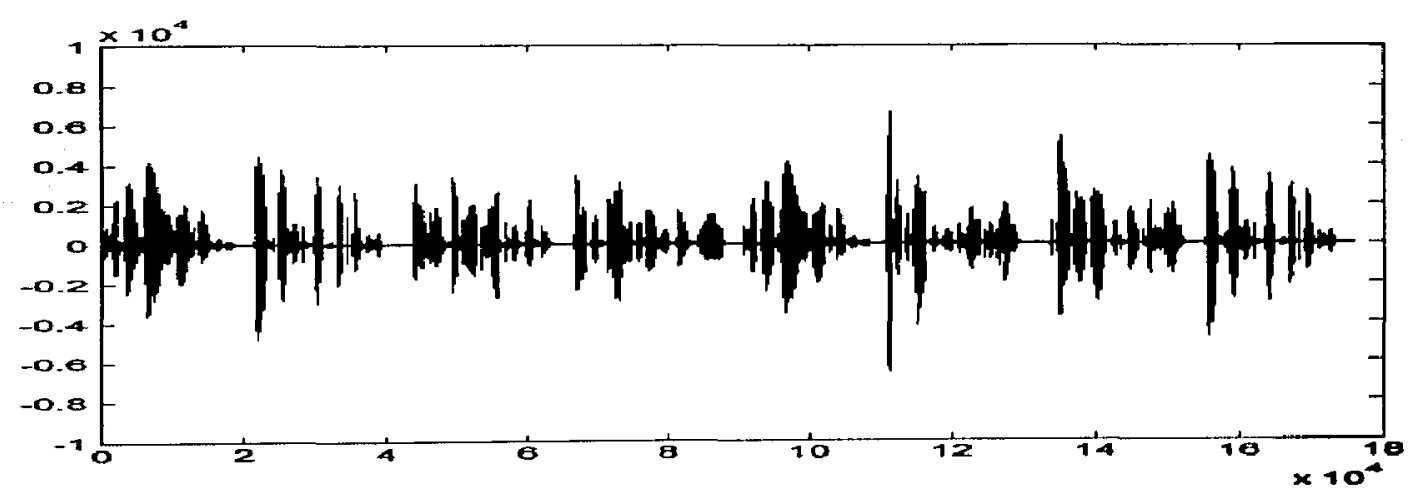

far end signal

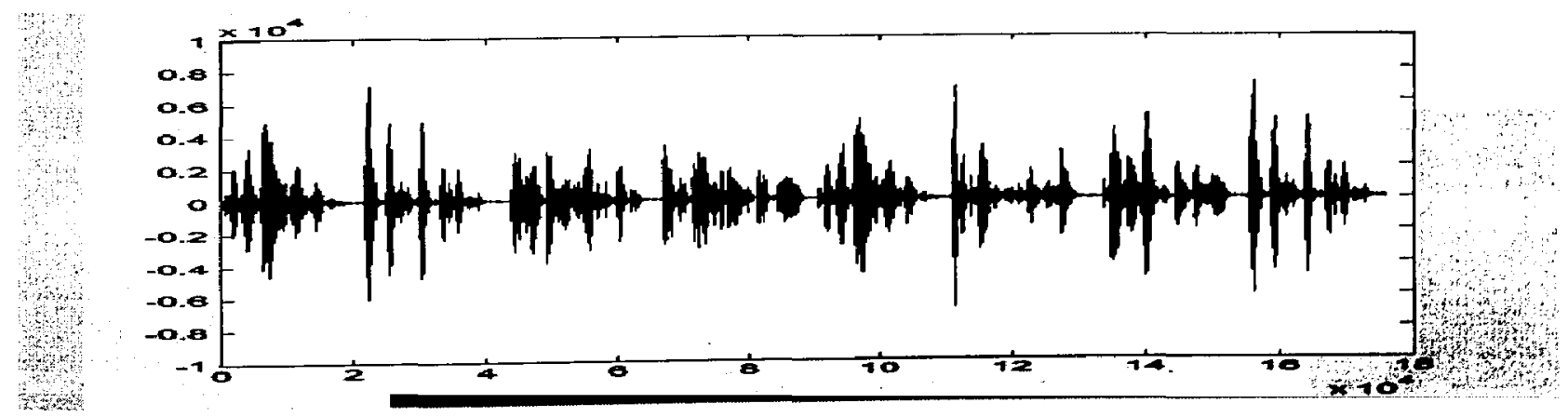

far end echoed signal 


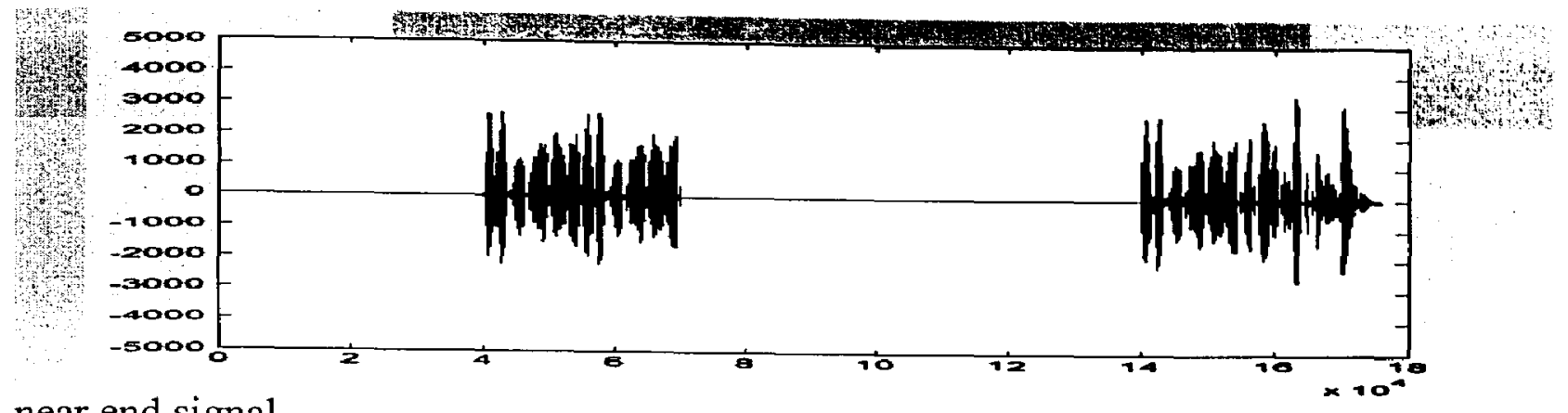

near end signal

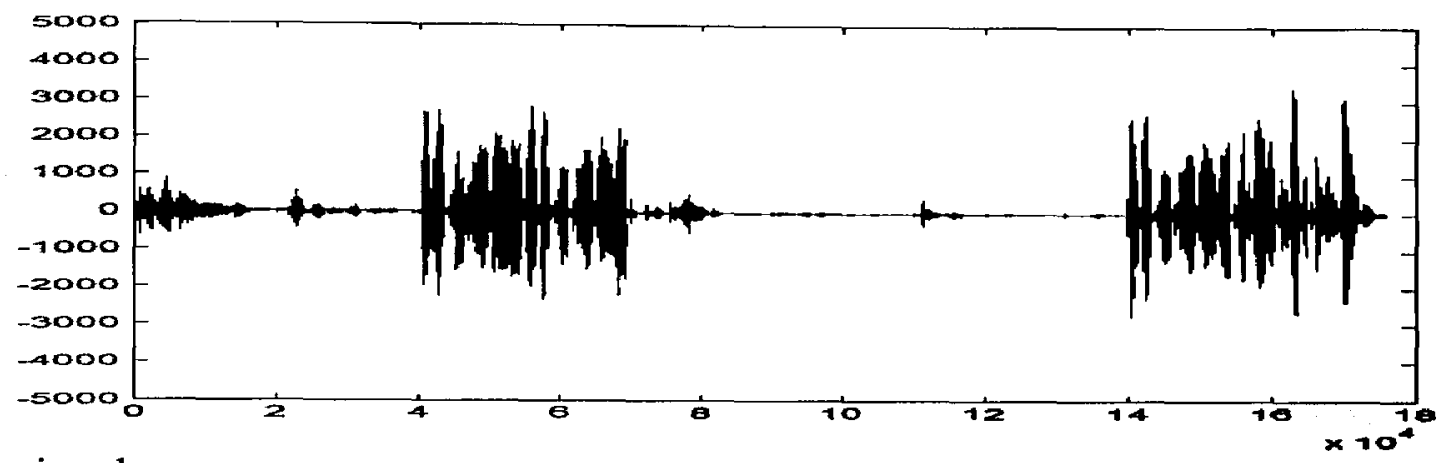

error signal

Figure 6.7 Test $3 \mathrm{C}$

\subsection{Test 4: Leak rate test}

The G.168 Requirements for Test 4: the requirements for the residual echo level are relaxed by $10 \mathrm{~dB}$ from the requirements for steady state in Test 1 and 2 .

\begin{tabular}{|l|l|l|}
\hline $\begin{array}{l}\text { IRin) } \\
\text { Input }\end{array}$ & $\begin{array}{l}\text { L(Ret) } \\
\text { Requirement (NLP Disabled) }\end{array}$ & Result \\
\hline$-10 \mathrm{dBm} 0$ & $-27 \mathrm{dBm} 0$ & $-25 \mathrm{dBm} 0$ \\
\hline
\end{tabular}

Table 6.10 Test 4 results

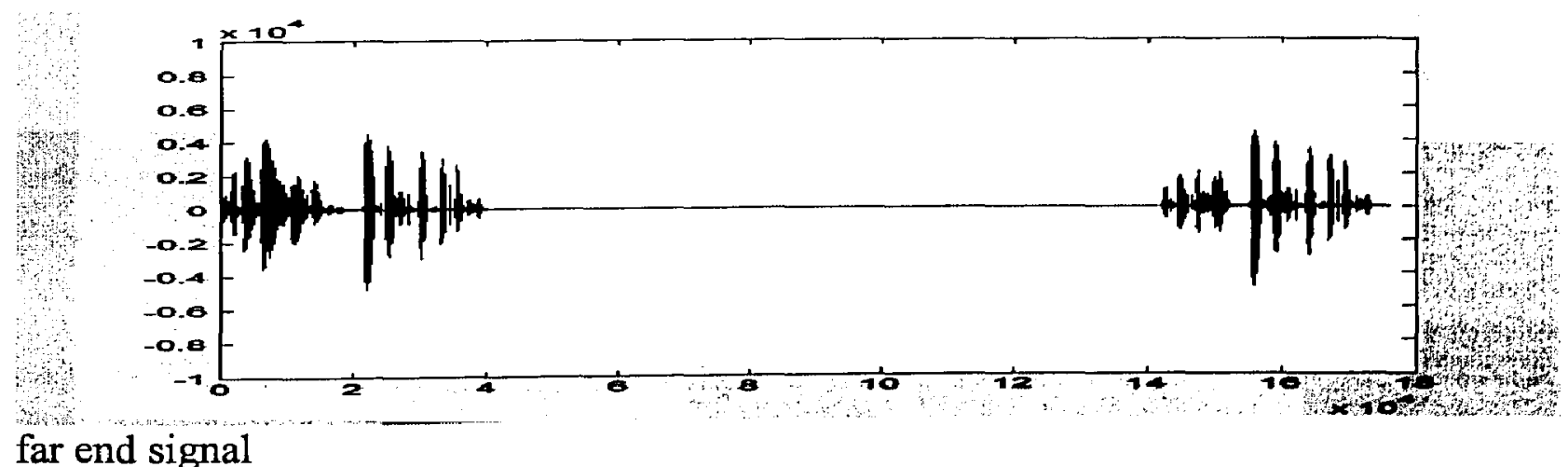




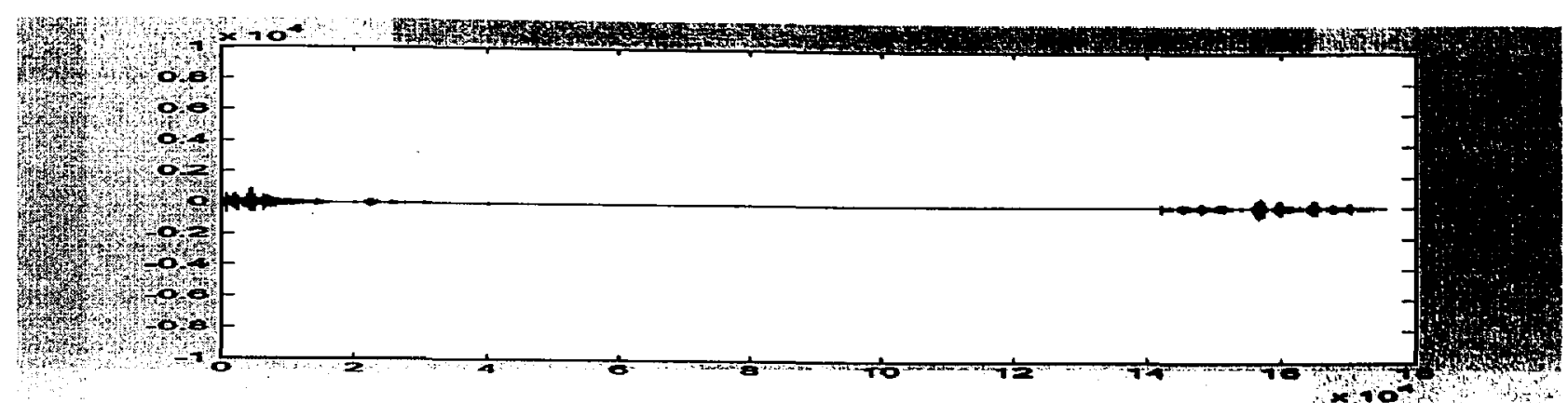

error signal

Figure 6.8 Test 4

\subsection{Test 5: Infinite return loss convergence test}

The G.168 Requirements for Test 5: the requirements are such that any spurious response vanishes to less than $20 \mathrm{~dB}$ below the input level by $1 \mathrm{sec}$ after the echo path is cut off and to less than 27 or $25 \mathrm{~dB}$ below the input level by $10 \mathrm{sec}$ after the echo path is cut off.

\begin{tabular}{|l|l|l|}
\hline $\begin{array}{l}\text { L(Rin) } \\
\text { Input }\end{array}$ & $\begin{array}{l}\text { L(Rin) - L(Res) } \\
\text { Requirement (NLP Disabled) }\end{array}$ & $\begin{array}{l}\text { Sample } \\
>20 \mathrm{~dB} \text { after } 1 \mathrm{sec} \\
>27 \mathrm{~dB} \text { after } 10 \mathrm{sec}\end{array}$ \\
\hline$-10 \mathrm{dBm} 0$ & meet req. \\
\hline
\end{tabular}

Table 6.11 Test 5 results

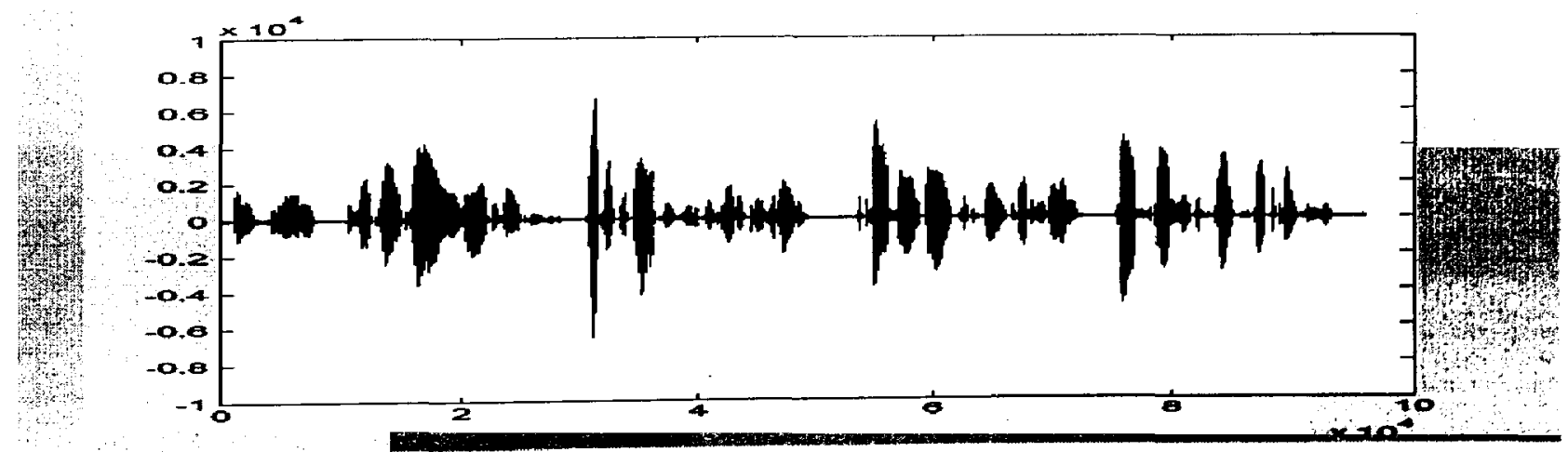

far end signal 

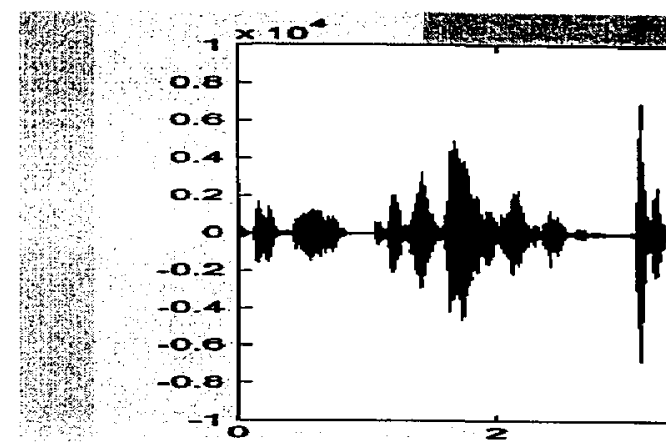

received signal

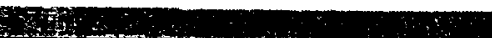

\subsection{Test 6: Non-divergence on narrow-band signals}

The G.168 Requirements for Test 6: the requirements for this test for the residual echo are relaxed by $10 \mathrm{~dB}$ versus the steady state residual echo requirements for Tests 1 and 2.

\begin{tabular}{|l|l|l|}
\hline W(Rin) & L(Ret) & $\begin{array}{l}\text { Result } \\
\text { (NLP Disabled) }\end{array}$ \\
\hline$-10 \mathrm{dBm} 0$ & $-27 \mathrm{dBm} 0$ & $-32 \mathrm{dBm} 0$ \\
\hline
\end{tabular}

Table 6.12 Test 6 results

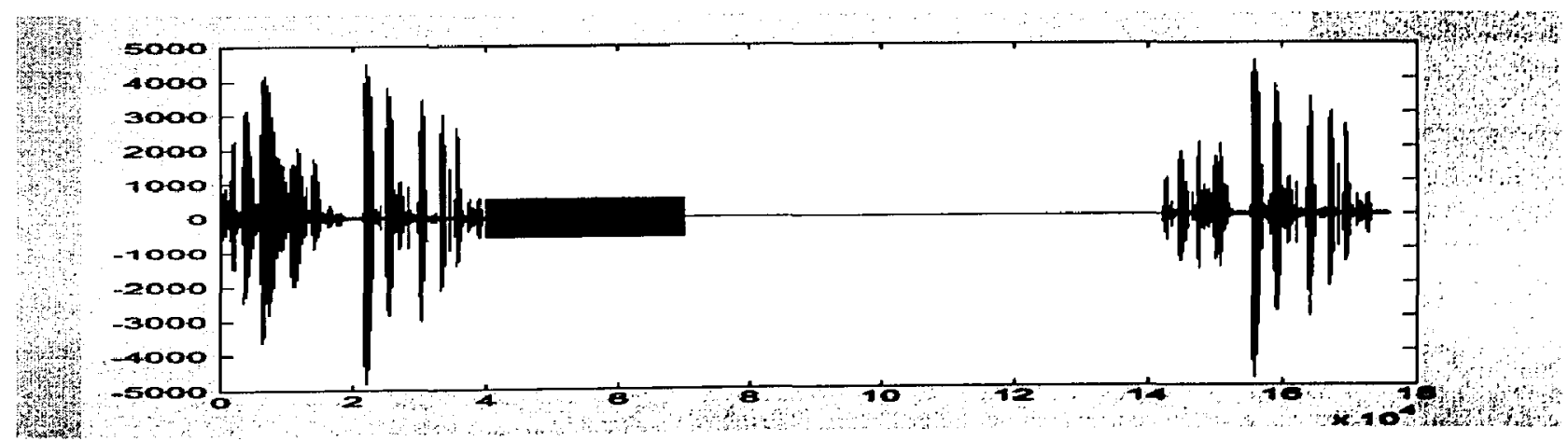

far end signal 


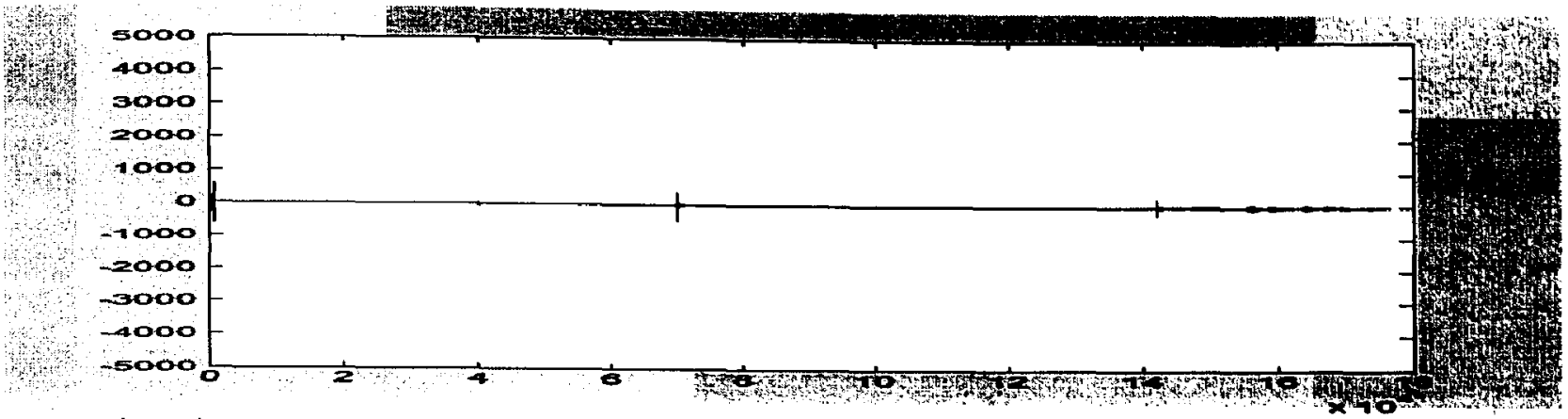

error signal

Figure 6.10 Test 6

\subsection{Test 7: Stability test}

The G.168 Requirements for Test 7: note that the requirements for this test are actually more stringent than that for steady state.

\begin{tabular}{|l|l|l|}
\hline $\begin{array}{l}\text { L(Rin) } \\
\text { Input }\end{array}$ & $\begin{array}{l}\text { L(Res), NLP dis } \\
\text { Output Requirement }\end{array}$ & $\begin{array}{l}\text { Result } \\
\text { (NLP Disableg) }\end{array}$ \\
\hline$-10 \mathrm{dBm} 0(1 \mathrm{kHz})$ & $-38 \mathrm{dBm} 0$ & $-48 \mathrm{dBm} 0$ \\
\hline
\end{tabular}

Table 6.13 Test 7 results

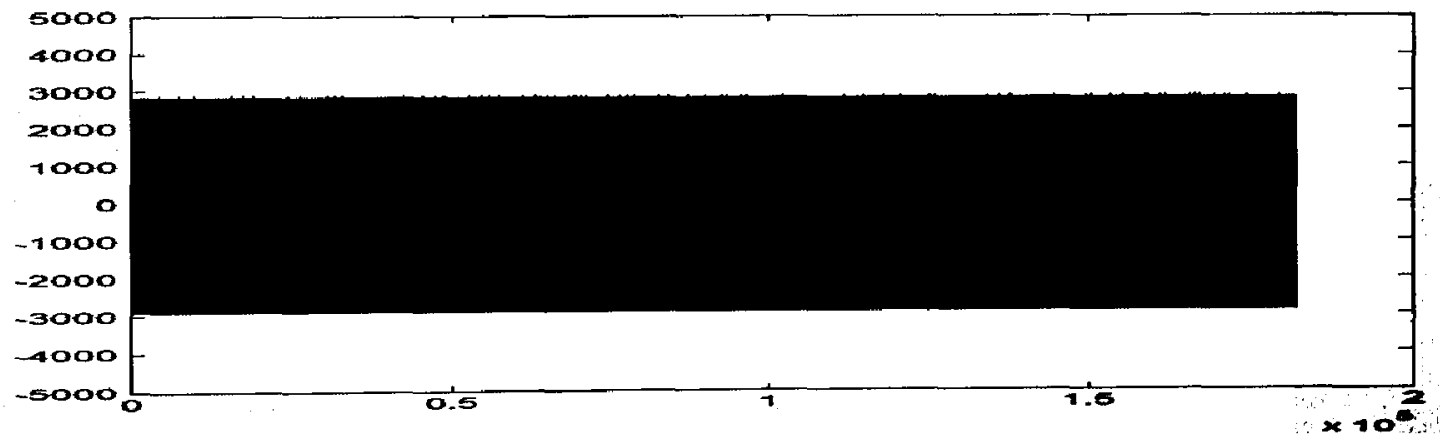

far end signal

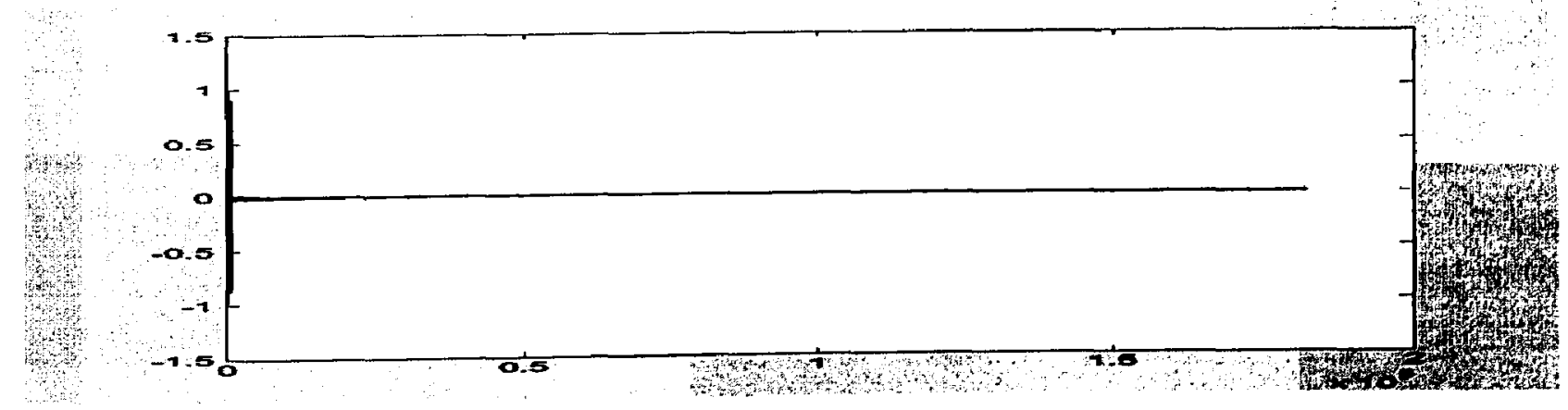

error signal

Figure 6.11 Test 7 


\subsection{Test 8: Non-convergence, in-band signaling, and continuity check tones}

The G.168 Requirements for Test 8: in this test, the signal applied at near end signal must remain uncancelled, while the echo of far end signal should be cancelled. The variation at filter output as compared to input should be within $\pm 2 \mathrm{~dB}$.

\begin{tabular}{|l|l|l|}
\hline Far end/near end Input & $\begin{array}{l}\text { Variation } \\
\text { Requirement }\end{array}$ & $\begin{array}{l}\text { Result } \\
\text { (NLP Enabled) }\end{array}$ \\
\hline $2400 / 2400$ & $\pm 2 \mathrm{~dB}$ & meet req. \\
\hline
\end{tabular}

Table 6.14 Test 8 results

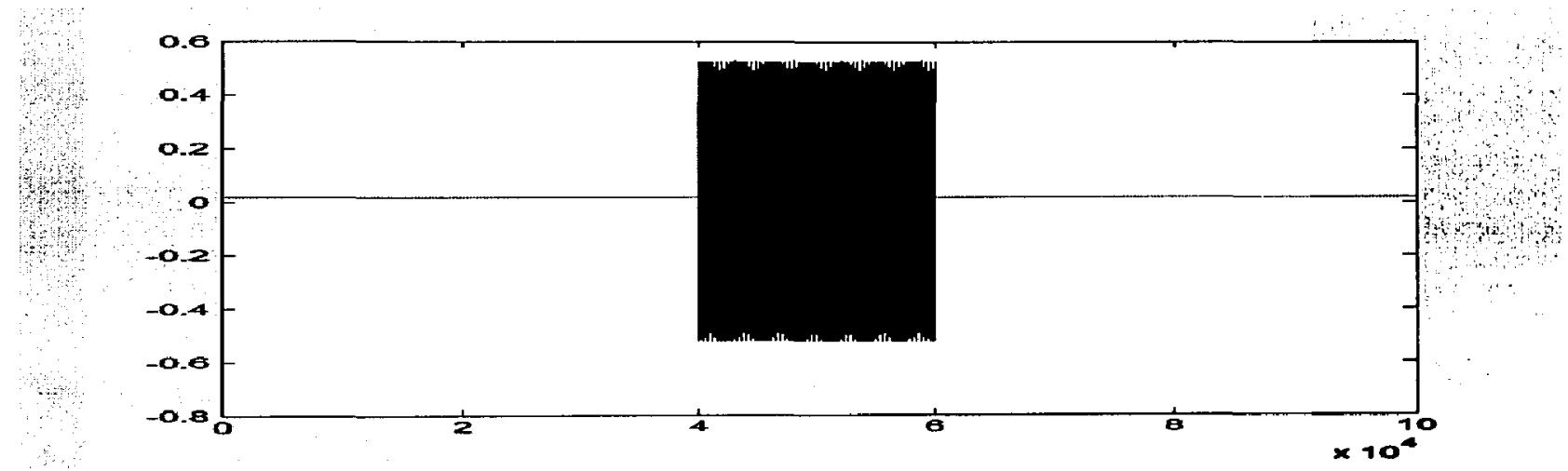

far end signal
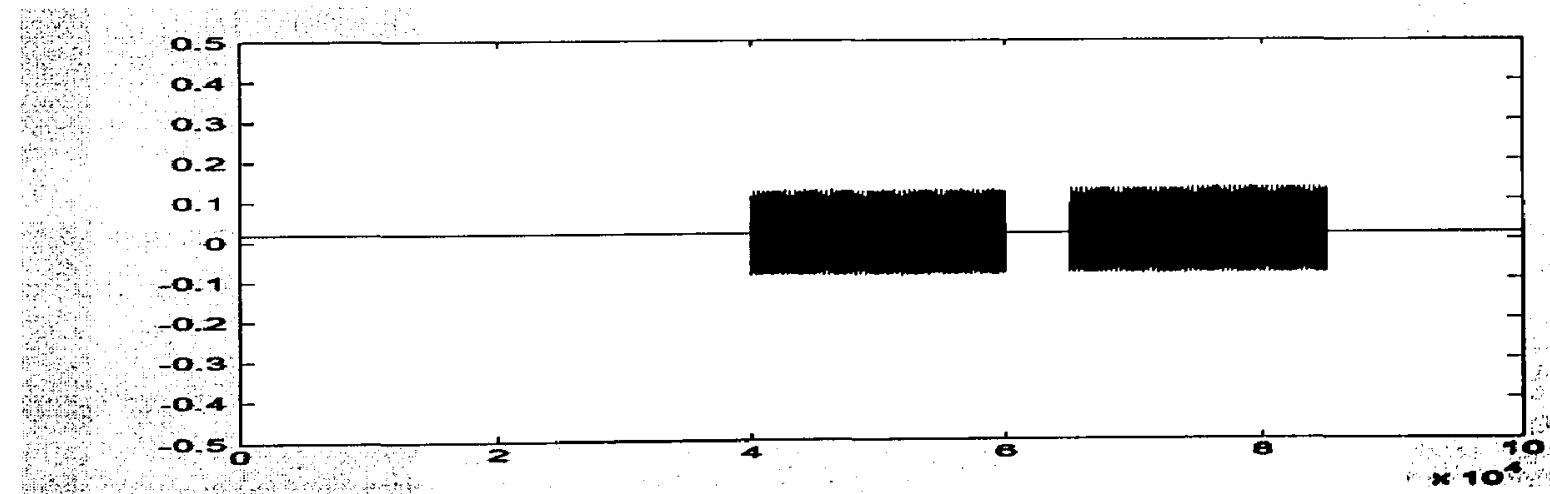

received signal 


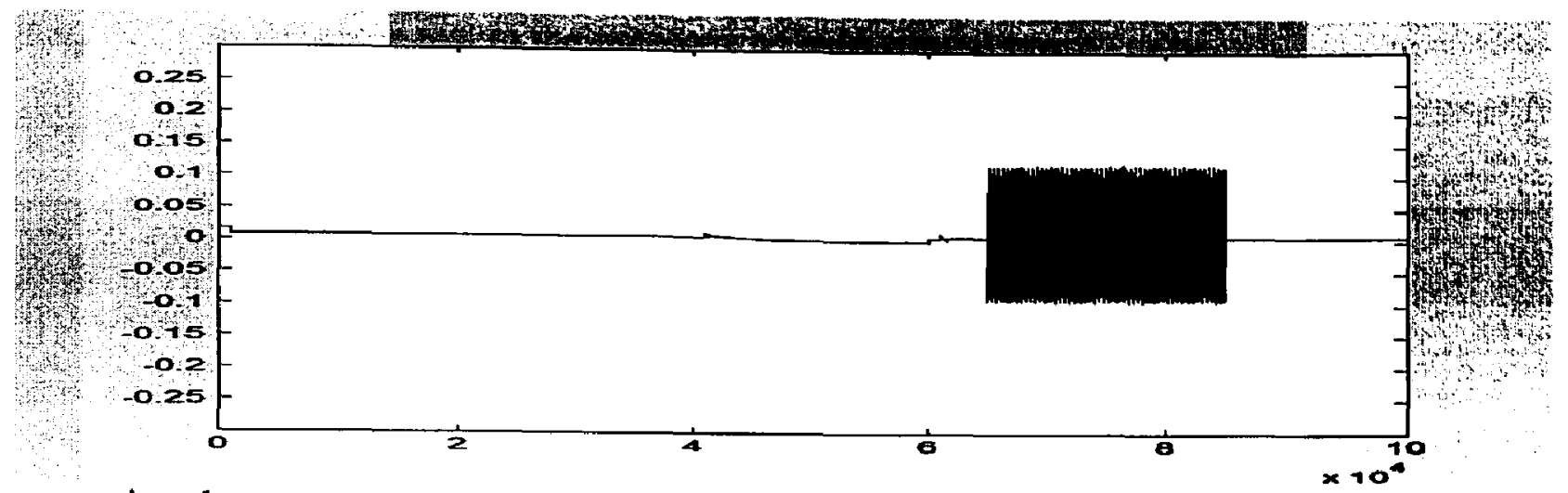

error signal

Figure 6.12 Test 8

\subsection{Summary}

Test 1: Steady state residual and residual echo level test

\begin{tabular}{|l|l|l|l|l|}
\hline $\begin{array}{l}\text { L(Rin) } \\
\text { Input }\end{array}$ & $\begin{array}{l}\text { L(Res), NLP Dis } \\
\text { Output Req. }\end{array}$ & Result & $\begin{array}{l}\text { L(Res), NLP enab } \\
\text { Output Req. }\end{array}$ & $\begin{array}{l}\text { Resurtit } \\
\text { (NLP Enabled) }\end{array}$ \\
\hline$-10 \mathrm{dBm} 0$ & $-37 \mathrm{dBm} 0$ & $-46.15 \mathrm{dBm} 0$ & $-65 \mathrm{dBm} 0$ & $-66.15 \mathrm{dBm} 0$ \\
\hline
\end{tabular}

Test 2A and 2B: Convergence test with NLP enabled and disabled

Test 2A

\begin{tabular}{|l|l|l|}
\hline Time (ms) & $\begin{array}{l}\text { L (Rin)-L (Ret) } \\
\text { (NLP Enabled) }\end{array}$ & $\begin{array}{l}\text { Result } \\
\text { (NLP Enabled) }\end{array}$ \\
\hline 0 & $6 \mathrm{~dB}$ & $48 \mathrm{~dB}$ \\
\hline $50 \mathrm{~ms}-1 \mathrm{~s}$ & $6 \mathrm{~dB} \sim 20 \mathrm{~dB}$ & $50 \mathrm{~dB}$ \\
\hline $1 \mathrm{~s}+$ & $($ - $-10 \mathrm{dBm} 055 \mathrm{~dB}$ & $57 \mathrm{~dB}$ \\
\hline
\end{tabular}

Test 2B

\begin{tabular}{|l|l|l|}
\hline Time & $\begin{array}{l}\text { L (Rin)-L (Ret) } \\
\text { (NLP Disabled) }\end{array}$ & $\begin{array}{l}\text { Result } \\
(\text { NLP Disabled) }\end{array}$ \\
\hline 0 & $6 \mathrm{~dB}$ & $8.8 \mathrm{~dB}$ \\
\hline $50 \mathrm{~ms}-1 \mathrm{~s}$ & $6 \mathrm{~dB} 20 \mathrm{~dB}$ & $9 \mathrm{~dB} \sim 20 \mathrm{~dB}$ \\
\hline $1 \mathrm{~s}-10 \mathrm{~s}$ & $20 \mathrm{~dB}$ & $40 \mathrm{~dB}$ \\
\hline $10 \mathrm{~s}+$ & $@-10 \mathrm{dBm} 027 \mathrm{~dB}$ & $42 \mathrm{~dB}$ \\
\hline
\end{tabular}


Test 2C: Convergence test in the presence of background noise

Test 2C(a)

\begin{tabular}{|l|l|l|l|}
\hline $\begin{array}{l}\text { L(Rin) } \\
\text { Input }\end{array}$ & $\begin{array}{l}\text { Noise Level } \\
\text { L(Rin)-15 }\end{array}$ & $\begin{array}{l}\text { L(Ret) } \\
\text { Requirement }\end{array}$ & $\begin{array}{l}\text { Result } \\
\text { (NLP Enabled) }\end{array}$ \\
\hline$-10 \mathrm{dBm} 0$ & $-30 \mathrm{dBm} 0$ & $-30 \mathrm{dBm} 0$ & $-46 \mathrm{dBm} 0$ \\
\hline
\end{tabular}

Test $2 \mathrm{C}(\mathrm{b})$

\begin{tabular}{|l|l|l|l|}
\hline $\begin{array}{l}\text { L(Rin) } \\
\text { Input }\end{array}$ & Noise Level & $\begin{array}{l}\text { L(Ret) } \\
\text { Requirement }\end{array}$ & $\begin{array}{l}\text { Result } \\
\text { (NLP Enabled) }\end{array}$ \\
\hline$-10 \mathrm{dBm} 0$ & $-55 \mathrm{dBm} 0$ & $-38 \mathrm{dBm} 0$ & $-46 \mathrm{dBm} 0$ \\
\hline
\end{tabular}

Test 2C(c)

\begin{tabular}{|l|l|l|l|}
\hline $\begin{array}{l}\text { L(Rin) } \\
\text { Input }\end{array}$ & $\begin{array}{l}\text { Noise Level } \\
\text { L(Rin)-15 }\end{array}$ & $\begin{array}{l}\text { L(Ret) } \\
\text { Requirement }\end{array}$ & $\begin{array}{l}\text { Sample } \\
\text { (NLP Disabled) }\end{array}$ \\
\hline$-10 \mathrm{dBm} 0$ & $-30 \mathrm{dBm} 0$ & $-30 \mathrm{dBm} 0$ & $-37 \mathrm{dBm} 0$ \\
\hline
\end{tabular}

Test 3: Performance under conditions of double talk

Test 3A

\begin{tabular}{|l|l|l|l|}
\hline $\begin{array}{l}\text { L(Rin) } \\
\text { Input }\end{array}$ & $\begin{array}{l}\text { Low Near-End } \\
\text { L(Rin)-15 }\end{array}$ & $\begin{array}{l}\text { Level L(Res) } \\
\text { Requirement }\end{array}$ & $\begin{array}{l}\text { Result } \\
\text { (NLP Disabled) }\end{array}$ \\
\hline$-10 \mathrm{dBm} 0$ & $-25 \mathrm{dBm} 0$ & $-25 \mathrm{dBm} 0$ & $-34 \mathrm{dBm} 0$ \\
\hline
\end{tabular}

Test 3B

\begin{tabular}{|l|l|l|l|}
\hline $\begin{array}{l}\text { L(Rin) } \\
\text { Input }\end{array}$ & $\begin{array}{l}\text { Doubletalk } \\
\mathbf{N} \geq \mathbf{L}(\text { Rin) }\end{array}$ & $\begin{array}{l}\text { Level L(Ret) } \\
\text { Requirement }\end{array}$ & $\begin{array}{l}\text { Result } \\
\text { (NLP Disabled) }\end{array}$ \\
\hline$-10 \mathrm{dBm} 0$ & $-10 \mathrm{dBm} 0$ & $-27 \mathrm{dBm} 0$ & $-35 \mathrm{dBm} 0$ \\
\hline
\end{tabular}

Test $3 \mathrm{C}$

\begin{tabular}{|l|l|l|l|}
\hline $\begin{array}{l}\text { L(Rin) } \\
\text { Input }\end{array}$ & $\begin{array}{l}\text { Doubletalk Level } \\
\text { N Performance }\end{array}$ & t2(Req.) & Result \\
\hline$-10 \mathrm{dBm} 0$ & $-10 \mathrm{dBm} 0$ & no peaks $>$ N & meets req. \\
\hline
\end{tabular}

\begin{tabular}{|l|l|l|l|}
\hline $\begin{array}{l}\text { t3(Req.) } \\
\text { Performance }\end{array}$ & Result & t4(Req.) & Result \\
\hline$-65 \mathrm{dBm} 0$ & $-52 \mathrm{dBm} 0$ & no peaks $>\mathrm{N}+6 \mathrm{dBm} 0$ & meets req. \\
\hline
\end{tabular}


Test 4: Leak rate test

\begin{tabular}{|l|l|l|}
\hline $\begin{array}{l}\text { L(Rin) } \\
\text { Input }\end{array}$ & $\begin{array}{l}\text { L(Ret) } \\
\text { Requirement (NLP Disabled) }\end{array}$ & Result $\mathrm{g}_{\mathrm{B}}$ \\
\hline$-10 \mathrm{dBm} 0$ & $-27 \mathrm{dBm} 0$ & $-25 \mathrm{dBm} 0$ \\
\hline
\end{tabular}

Test 5: Infinite return loss convergence test

\begin{tabular}{|l|l|l|}
\hline $\begin{array}{l}\text { L(Rin) } \\
\text { Input }\end{array}$ & $\begin{array}{l}\text { L(Rin) }- \text { L(Res) } \\
\text { Requirement (NLP Disabled) }\end{array}$ & Sample \\
\hline$-10 \mathrm{dBm} 0$ & $>20 \mathrm{~dB}$ after $1 \mathrm{sec}$ \\
$>27 \mathrm{~dB}$ after $10 \mathrm{sec}$ & meet req. \\
\hline
\end{tabular}

Test 6: Non-divergence on narrow-band signals

\begin{tabular}{|l|l|l|}
\hline L(Rin) & $\begin{array}{l}\text { L(Ret) } \\
\text { Final Requirement }\end{array}$ & $\begin{array}{l}\text { Result } \\
\text { (NLP Disabled) }\end{array}$ \\
\hline$-10 \mathrm{dBm} 0$ & $-27 \mathrm{dBm} 0$ & $-32 \mathrm{dBm} 0$ \\
\hline
\end{tabular}

Test 7: Stability test

\begin{tabular}{|l|l|l|}
\hline $\begin{array}{l}\text { L(Rin) } \\
\text { Input }\end{array}$ & $\begin{array}{l}\text { L(Res), NLP dis } \\
\text { Output Requirement }\end{array}$ & $\begin{array}{l}\text { Result } \\
\text { (NLP Disabled) }\end{array}$ \\
\hline$-10 \mathrm{dBm} 0(1 \mathrm{kHz})$ & $-38 \mathrm{dBm} 0$ & $-48 \mathrm{dBm} 0$ \\
\hline
\end{tabular}

Test 8: Non-convergence, in-band signaling, and continuity check tones

\begin{tabular}{|l|l|l|}
\hline Far end/near end Input & $\begin{array}{l}\text { Variation } \\
\text { Requirement }\end{array}$ & $\begin{array}{l}\text { Result } \\
\text { (NLP Enabled) }\end{array}$ \\
\hline $2400 / 2400$ & $\pm 2 \mathrm{~dB}$ & meet req. \\
\hline
\end{tabular}

Table 6.15 Test result summary 


\section{Chapter 7}

\section{Real time simulation}

\subsection{TI TMS320C6711 DSK}

The TI TMS320 floating-point family includes C3x, C4x, and C67x. Each generation of the TMS320 series has a unique central processing unit (CPU) with a variety of memory and peripheral configurations. In this project, the TMS320C6711 DSK is chosen to the real-time AEC simulations.

The TI TMS320C6711 DSK is a digital signal processing development kit used to prototype DSP applications targeted for the C6711 family of processors. It has a $3.5-\mathrm{mm}$ audio IN jack and 3.5-mm audio OUT jack, with ADC and DAC executed onboard. The AD535 codec works at a fixed sample rate of $8 \mathrm{kHz}$. The DSK also includes $16 \mathrm{MB}$ of synchronous dynamic RAM and $128 \mathrm{~KB}$ flash ROM and the 150-MHz C6711DSP is capable of executing 900 million floating-point operations per second (MFLOPS).

The DSK board is connected to a PC via a parallel port. The program files can be created in TI code composer studio on the PC, and then loaded onto the DSK [16].

\subsection{C6711 DSK Simulation using Code Composer Studio}

\subsubsection{Simulation Procedure Introduction}

CCS IDE from TI is easy to use development environment allows DSP designers of all experience levels to move quickly through each phase of the application development process including design, code and build, debug, analyze and optimize. The fully integrated development environment includes, real-time analysis capabilities, easy to use 
debugger, $\mathrm{C} / \mathrm{C}++\mathrm{Compiler,} \mathrm{Assembler,} \mathrm{linker,} \mathrm{editor,} \mathrm{visual} \mathrm{project} \mathrm{manager,} \mathrm{simulators,}$ XDS560 and XDS510 emulation drivers and DSP/BIOS support.

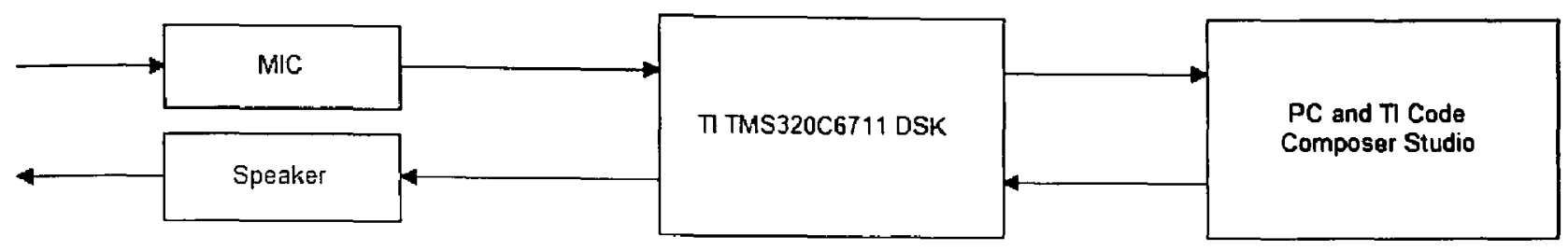

Figure 7.1 Adaptive echo cancellation implementation block diagram

The real-time acoustic echo cancellation system has been implemented as shown in figure 8.1. A microphone is used to input a voice signal from the user, this is then fed into the Texas Instrument TMS320C6711 development board. Based on human voice frequency range and adaptive echo cancellation system performance consideration, the sampling rate $8 \mathrm{KHz}$ is used in the $\mathrm{AD} 535$ codec. The echo cancellation system use the normalized LMS algorithm and the normalized cross-correlation DTD algorithm detailed in previous sections. At each new sample time the input is sampled and input to memory and these memory audio data can be output to a file. In this project, there are two audio data files that contain these samples are loaded into memory as far end signal and received signal, and the CCS development workbench window is show in the Figure 8.2. The step size value for this iteration of the NLMS algorithm is then calculated by inverting the dot product of the input vector and a small constant should be included in the denominator to avoid zero divides. The output of the adaptive filter is then calculated by the dot product of the input vector and the current filter tap weight vector. This output is then subtracted from the desired signal to determine the estimation error value, e(n). This error value, the step size value and the current FIR tap weight vector are then input to the NLMS algorithm to calculate the filters tap weight to be used in the next iteration. If the filter is 
claimed to converge, the DTD detector will start monitor if there is a double talk situation. When double talk happens, the DTD detector inhibits the filter adaptation for a hold time to avoid divergence of the adaptive algorithm. Finally the AEC output error signal data is streamed out to a specified file using File I/O function provided by CCS to do further analysis [13][16][17].

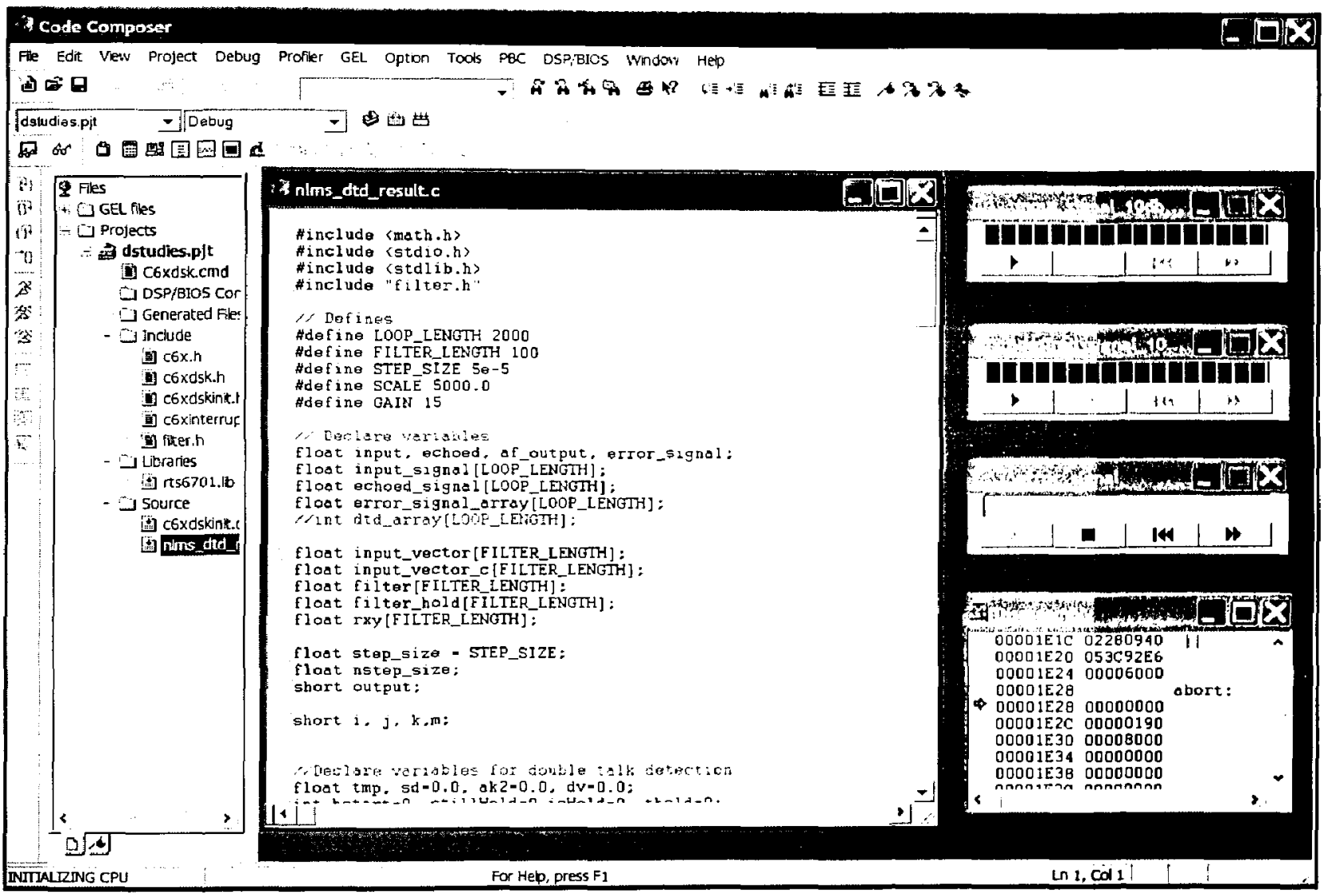

Figure 7.2 CCS Workbech window

\subsubsection{C6711 DSK Simulation Results}

Using the CCS graphing function provided by CSS integration development environment, the far end signal, received signal and the error signal of the echo cancellation system can be viewed and shown in the following figures. As can be seen that the real time adaptive echo cancellation system is successfully developed with the NLMS and the normalized cross-correlation DTD algorithm. 
(1) Steady state residual and residual echo level test

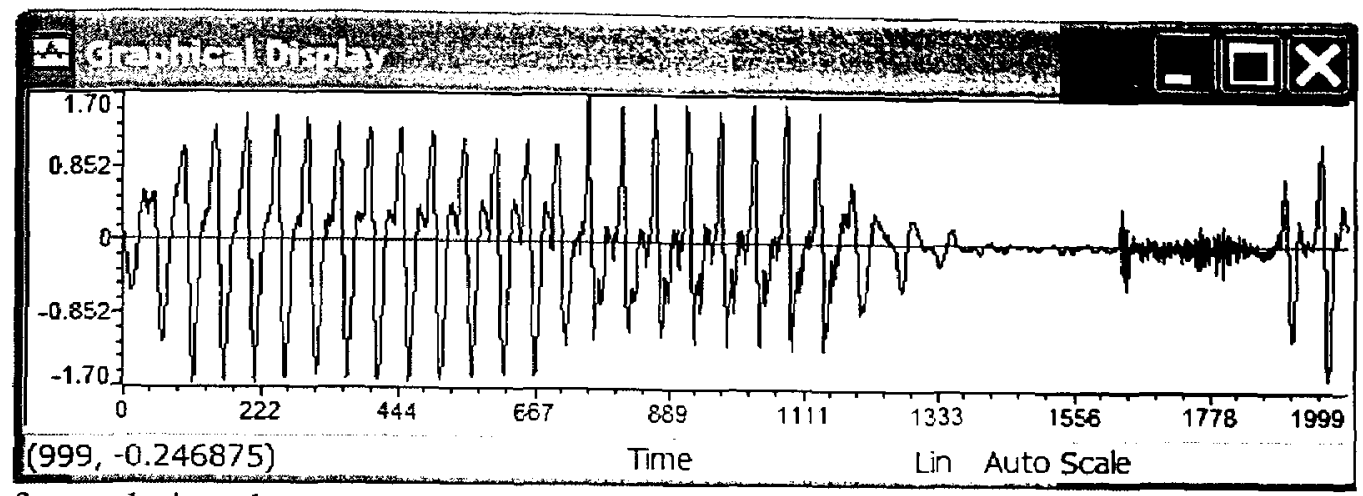

far end signal

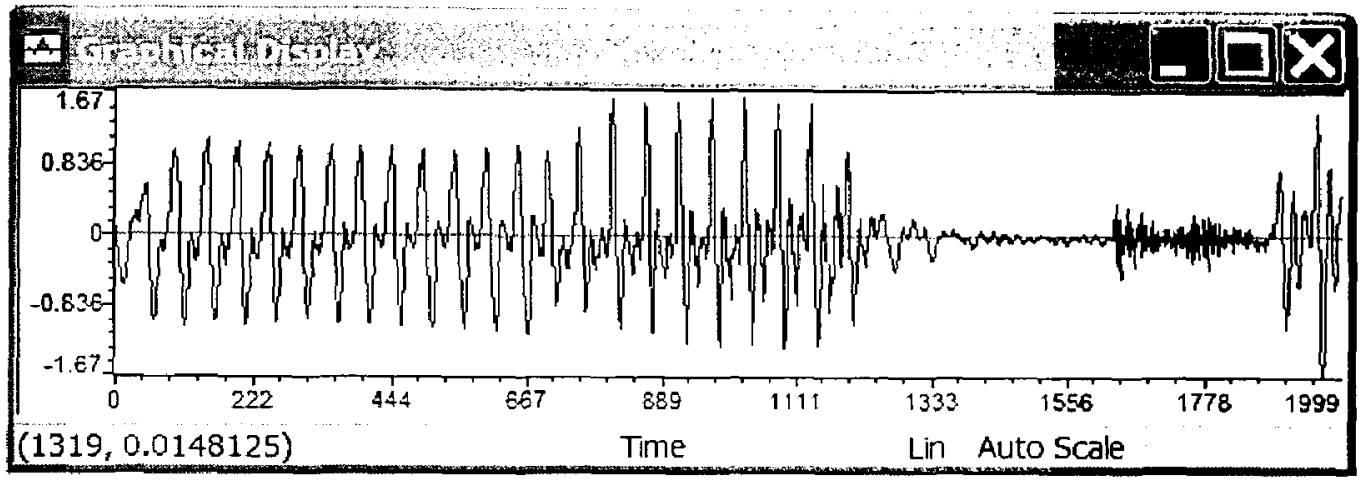

received signal

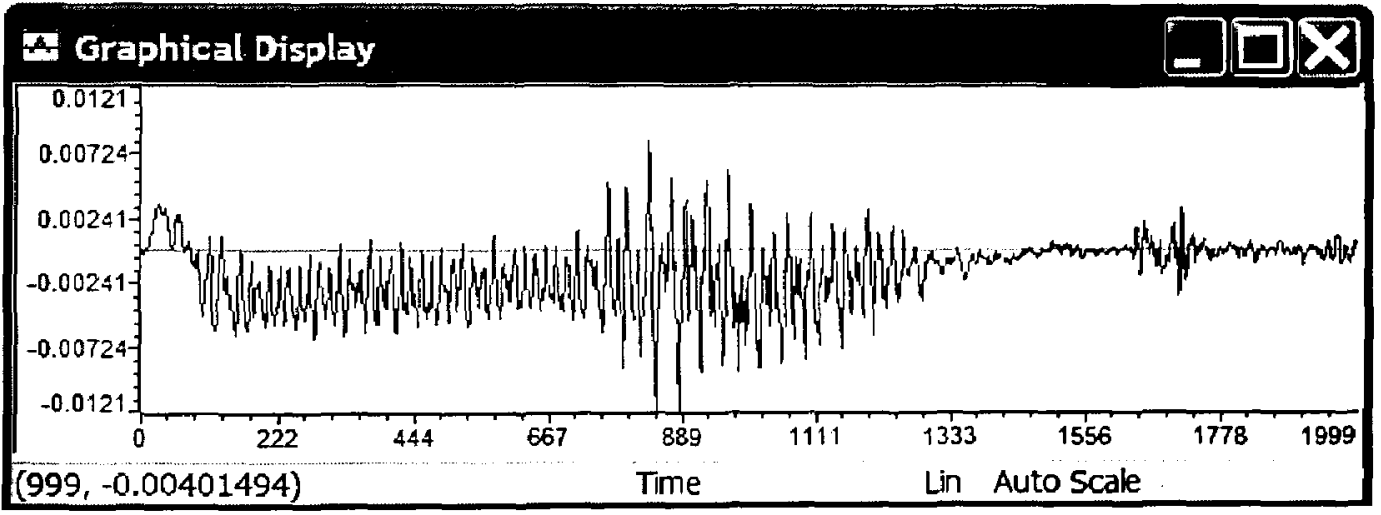

error signal

Figure 7.3 Steady state residual and residual echo level test

\begin{tabular}{|c|c|c|c|}
\hline Input & Output Req. & Result & Matlab Rerult to \\
\hline$-10 \mathrm{dBm} 0$ & $-37 \mathrm{dBm} 0$ & $-44 \mathrm{dBm} 0$ & $-46 \mathrm{dBm} 0$ \\
\hline
\end{tabular}

Table 7.1 Steady state residual and residual echo level test result 
(2) Convergence test in the presence of background noise

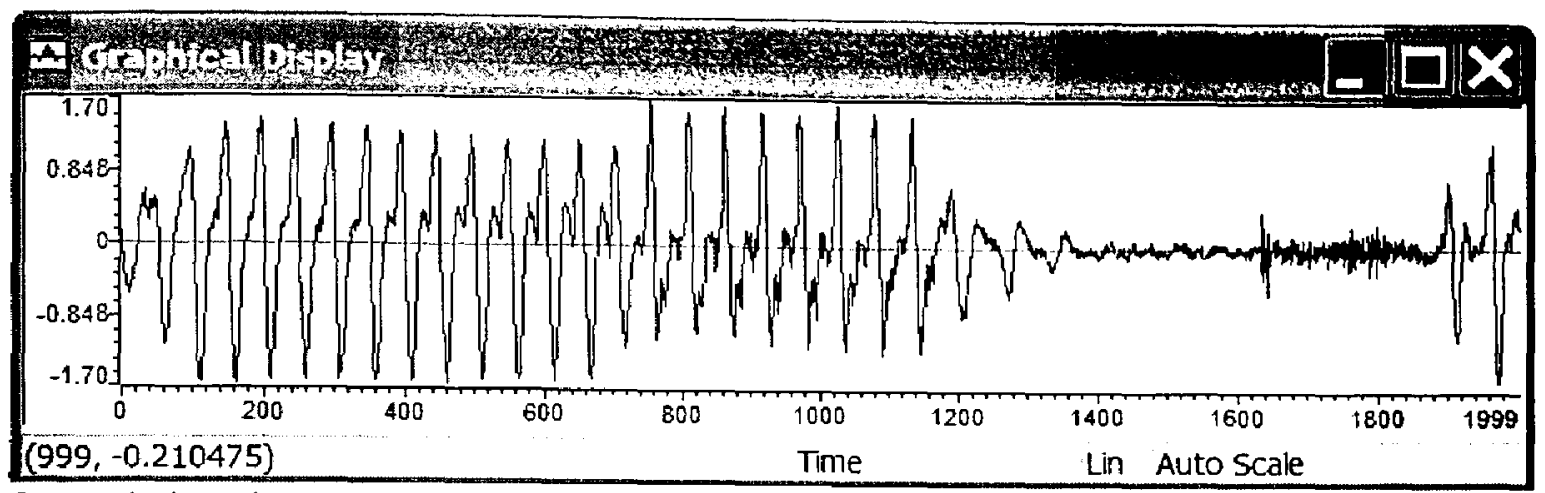

far end signal

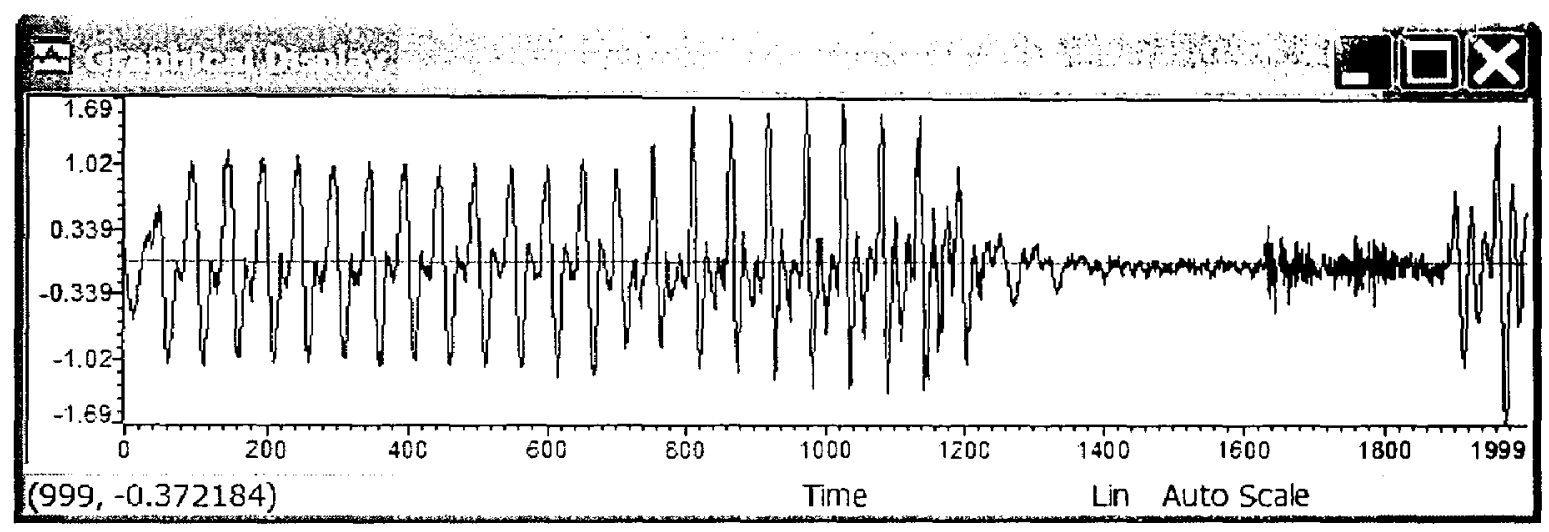

received signal

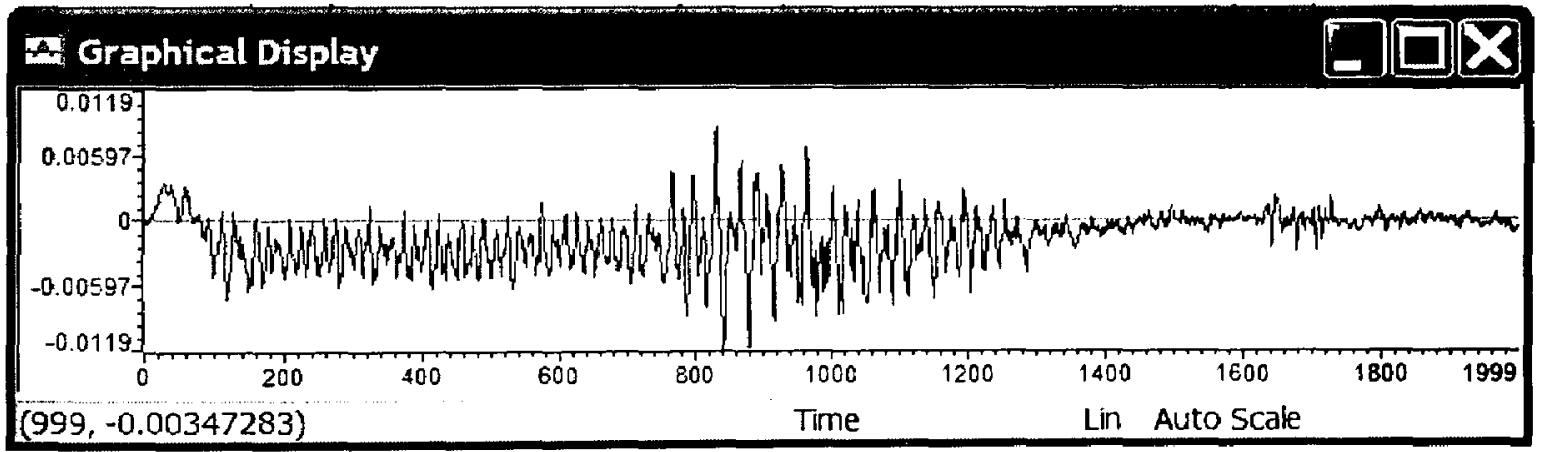

error signal

Figure 7.4 Convergence test in the presence of background noise

\begin{tabular}{|l|l|l|l|l|}
\hline Input & Noise & Output Req. & Matlab Result & Result \\
\hline$-10 \mathrm{dBm} 0$ & $-30 \mathrm{dBm} 0$ & $-30 \mathrm{dBm} 0$ & $-46 \mathrm{dBm} 0$ & $-44 \mathrm{dBm} 0$ \\
\hline
\end{tabular}

Table 7.2 Convergence test in the presence of background noise test results 
(3) Double talk test with low near end levels

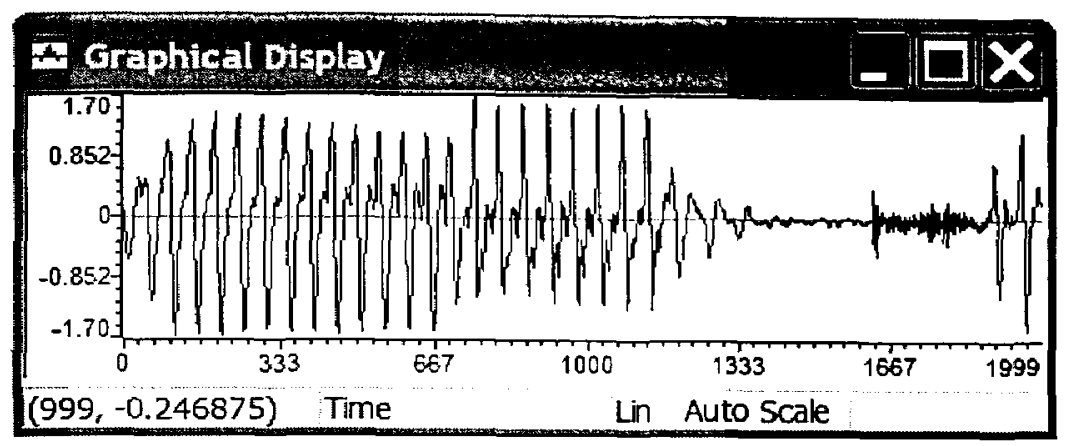

far end signal

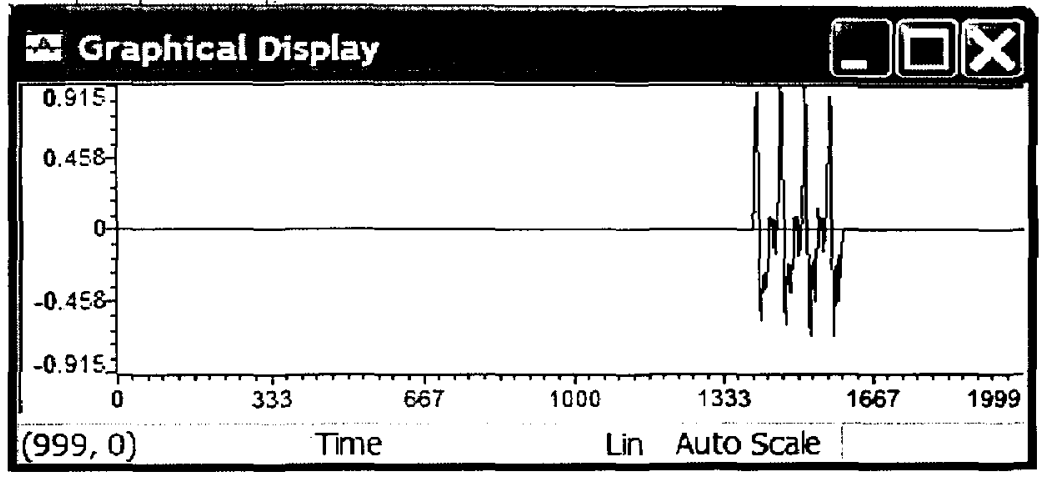

near signal

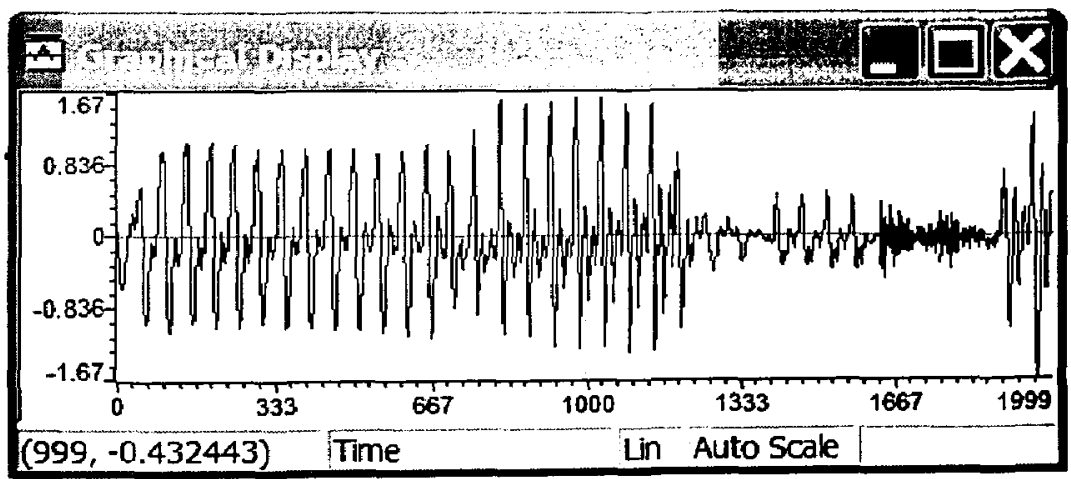

received signal 


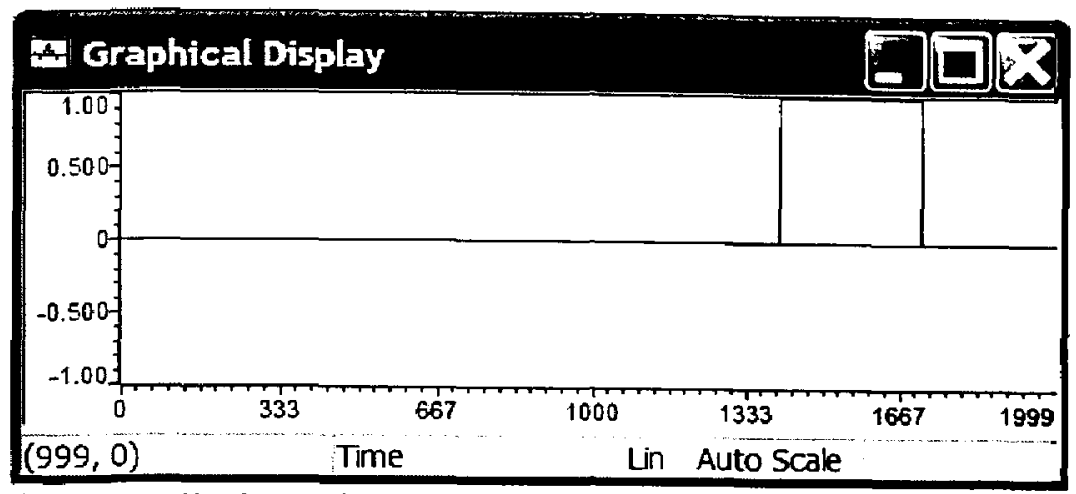

double talk detection

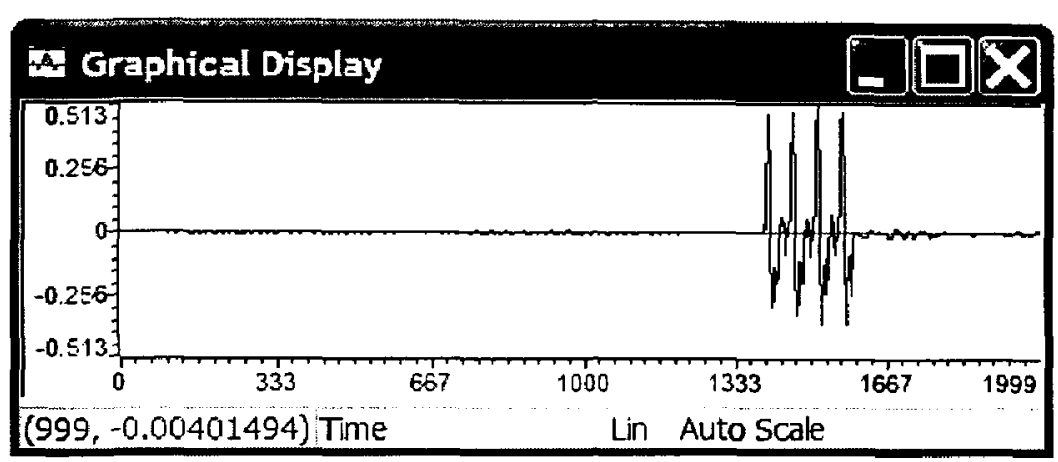

error signal

Figure 7.5 Double talk test with low near end levels

\begin{tabular}{|l|l|l|l|l|}
\hline Input & Near end & Output Req. & Matlab Result & Recsuif \\
\hline$-10 \mathrm{dBm} 0$ & $-25 \mathrm{dBm} 0$ & $-25 \mathrm{dBm} 0$ & $-34 \mathrm{dBm} 0$ & $-33 \mathrm{dBm} 0$ \\
\hline
\end{tabular}

Table 7.3 Double talk test with low near end levels test results

(4) Double talk test with high near end levels

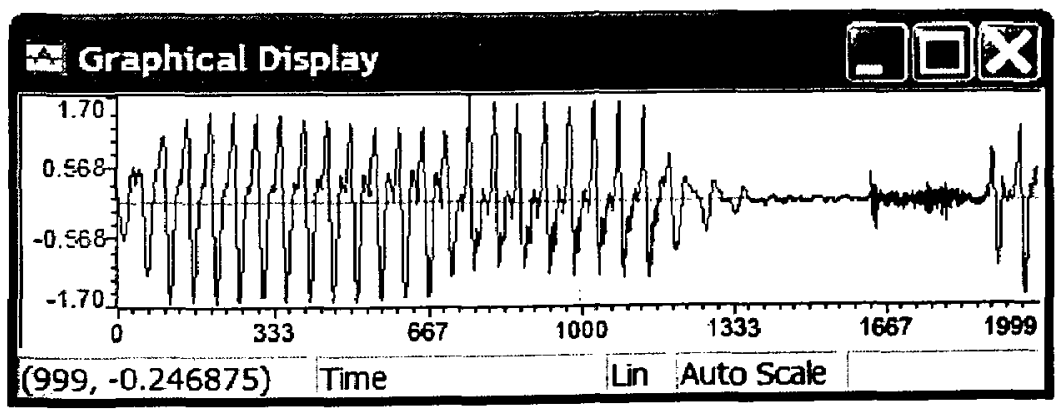

far end signal 


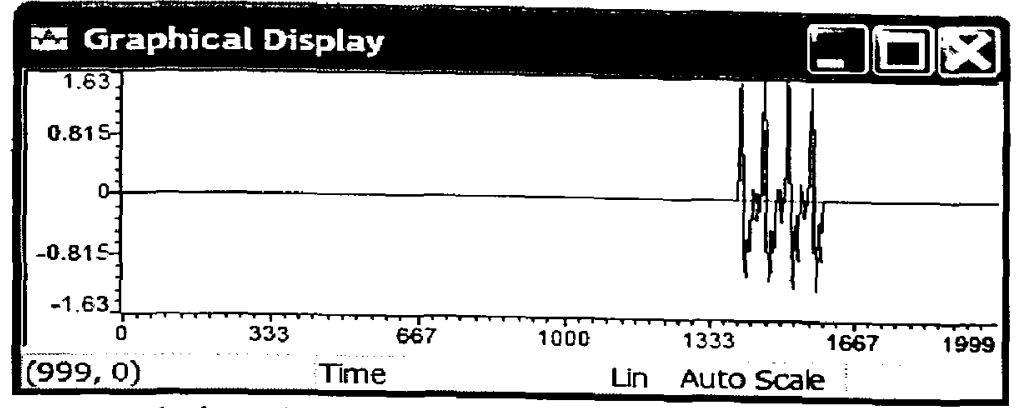

near end signal

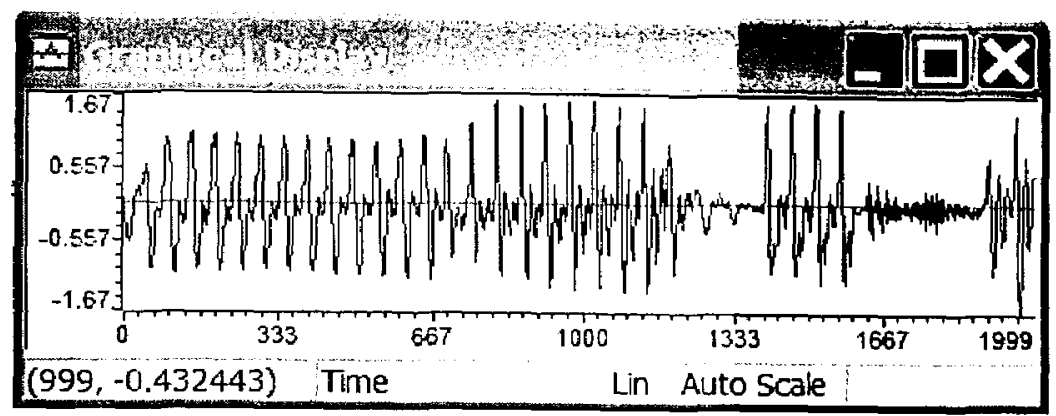

received signal

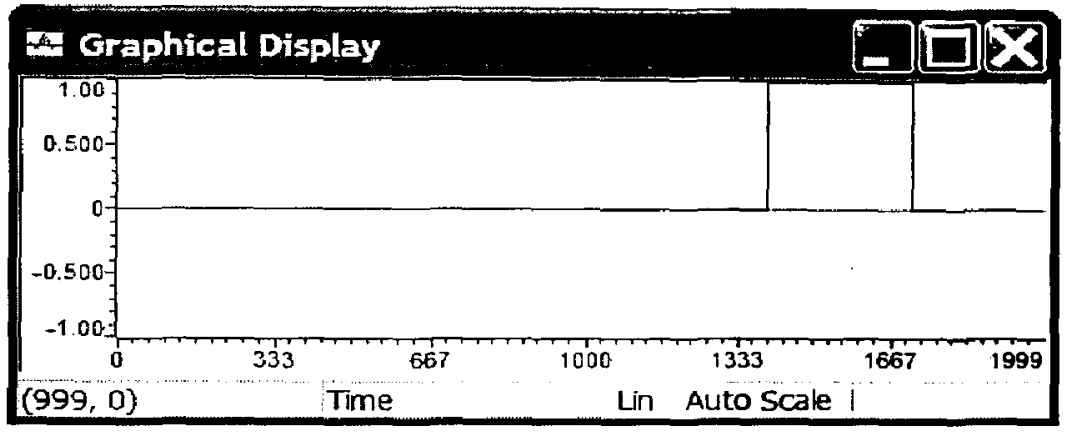

double talk detecion

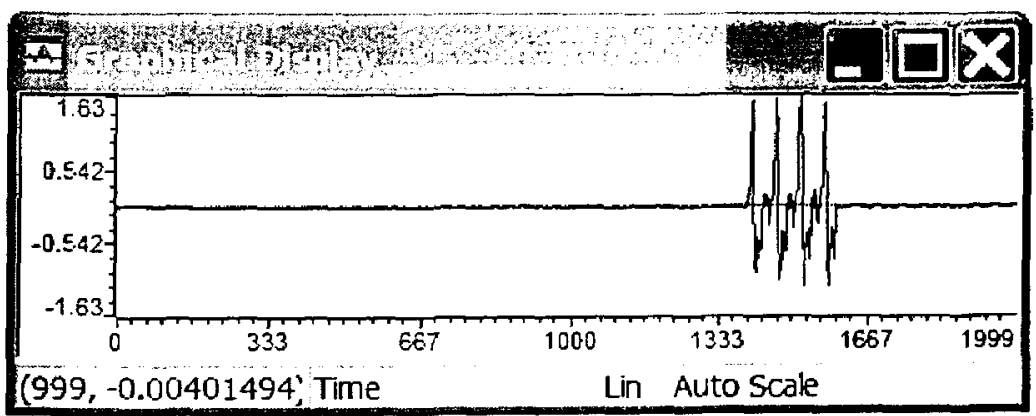

error signal

Figure 7.6 Double talk test with high near end levels

\begin{tabular}{|l|l|l|l|l|}
\hline Input & Near end & Output Req. & Matlab Result & F \\
\hline$-10 \mathrm{dBm} 0$ & $-10 \mathrm{dBm} 0$ & $-27 \mathrm{dBm} 0$ & $-35 \mathrm{dBm} 0$ & $-30 \mathrm{dBm} 0$ \\
\hline
\end{tabular}

Table 7.4 Double talk test with high near end levels test results 
(5) Non-divergence on narrow-band signals

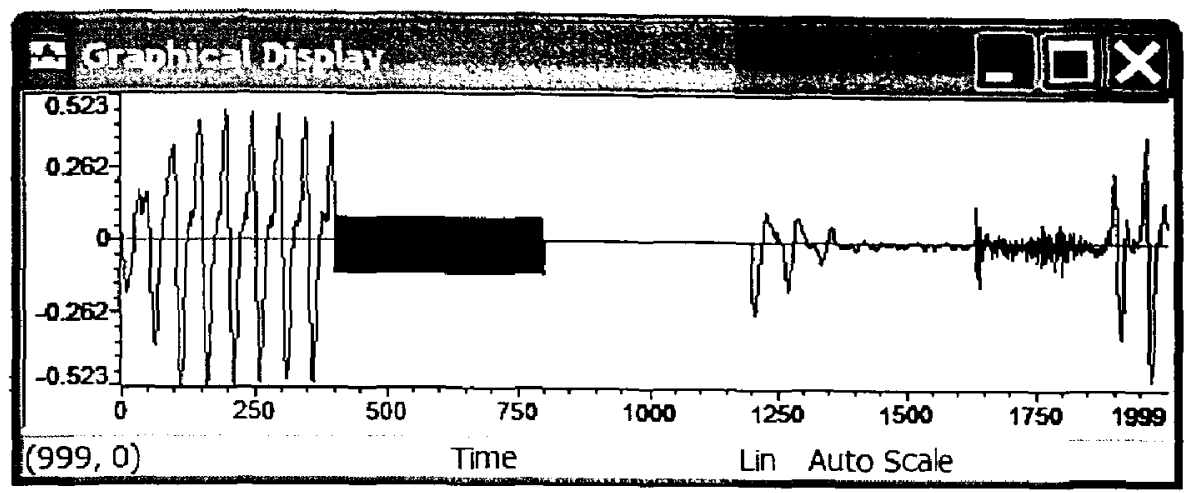

far end signal

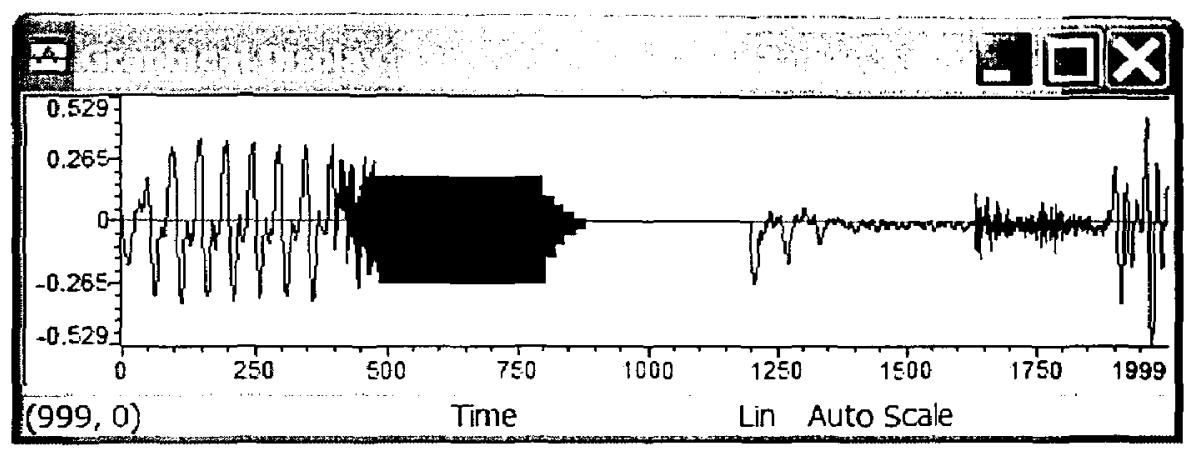

received signal

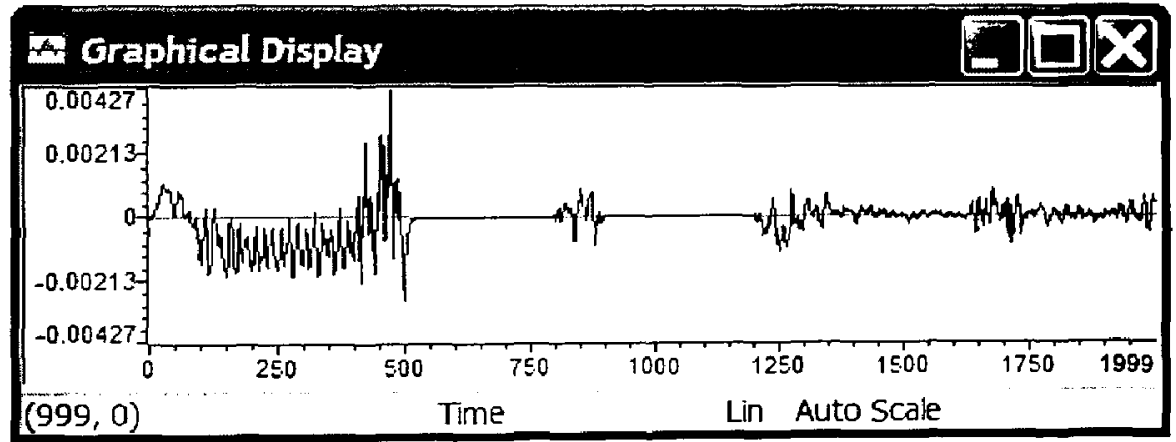

error signal

Figure 7.7 Non-divergence on narrow-band signals

\begin{tabular}{|l|l|l|l|}
\hline Input & Output Req. & Matlab Result & Resulted \\
\hline$-10 \mathrm{dBm} 0$ & $-27 \mathrm{dBm} 0$ & $-32 \mathrm{dBm} 0$ & $-34 \mathrm{dBm} 0$ \\
\hline
\end{tabular}

Table 7.5 Non-divergence on narrow-band signals test result 
(6) Stability test

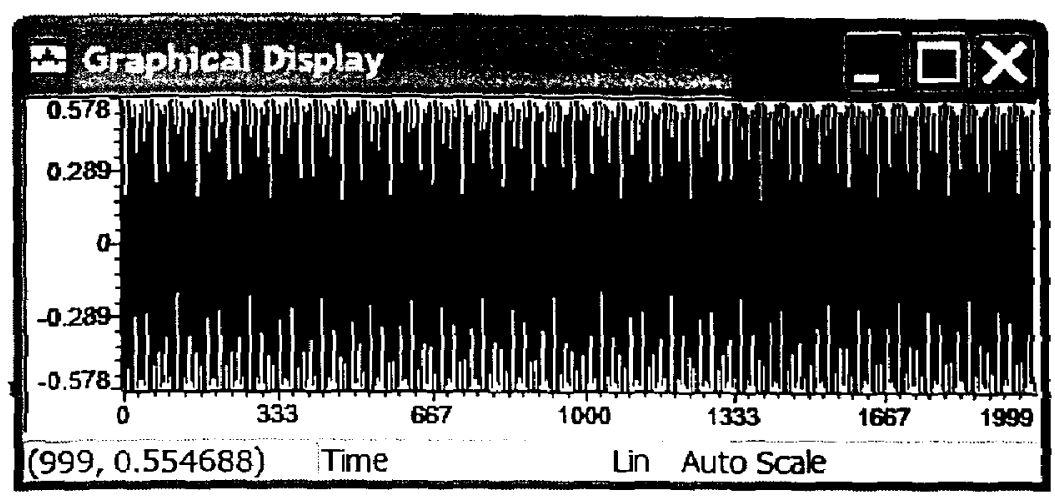

far end signal

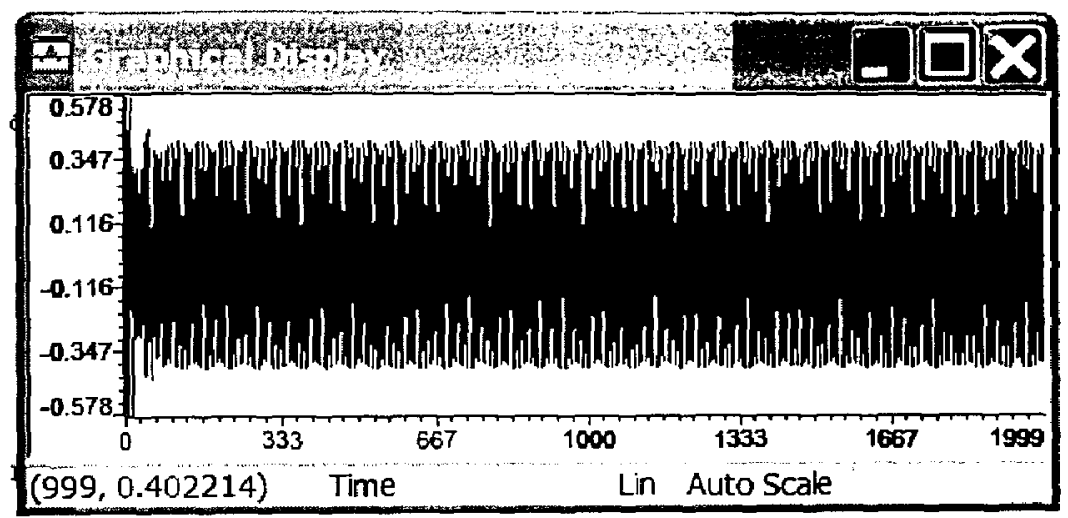

received signal

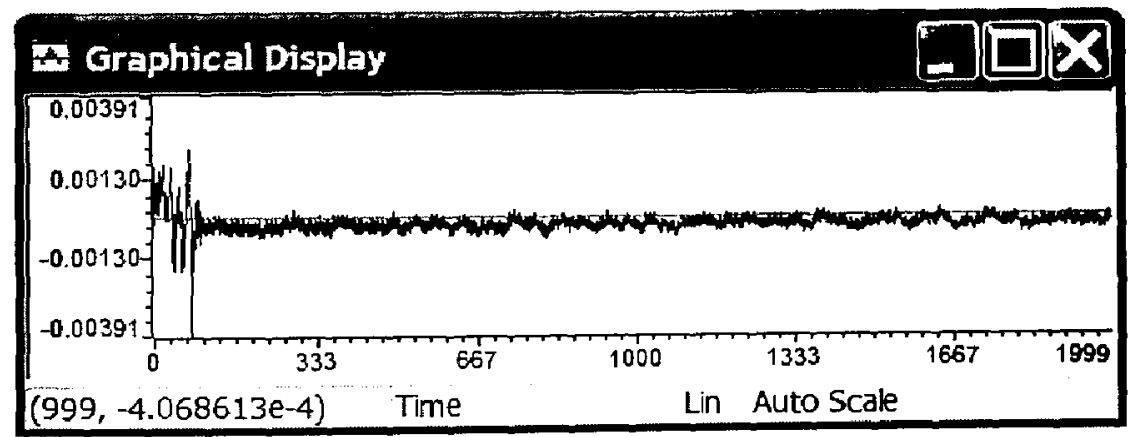

error signal

Figure 7.8 Stability test

\begin{tabular}{|l|l|l|l|}
\hline Input & Output Req. & Matlab Reswit & Kegin \\
\hline$-20 \mathrm{dBm} 0$ & $-47 \mathrm{dBm} 0$ & $-48 \mathrm{dBm} 0$ & $-58 \mathrm{dBm} 0$ \\
& & $@-10 \mathrm{dBm} 0$ input & \\
\hline
\end{tabular}

Table 7.6 Stability test results 
(7) Non-convergence, in-band signaling, and continuity check tones

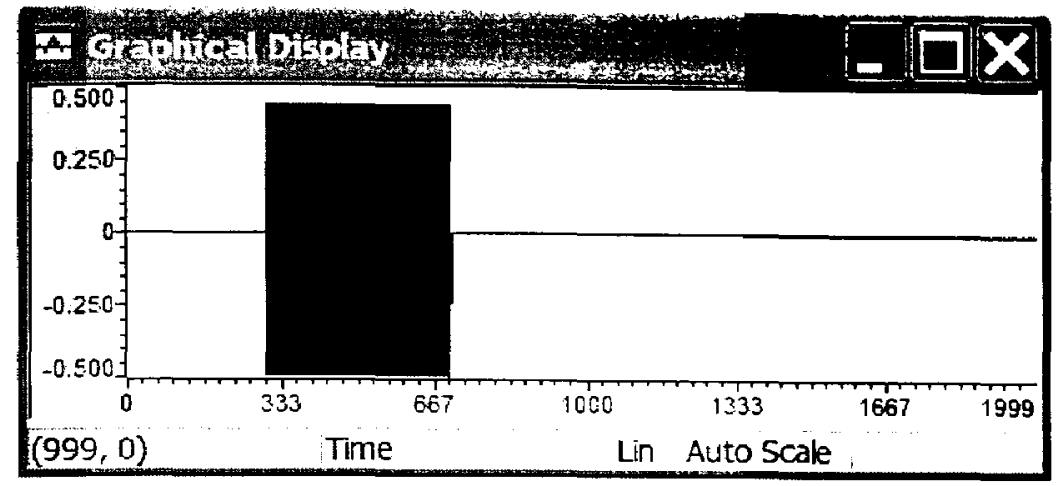

far end signal

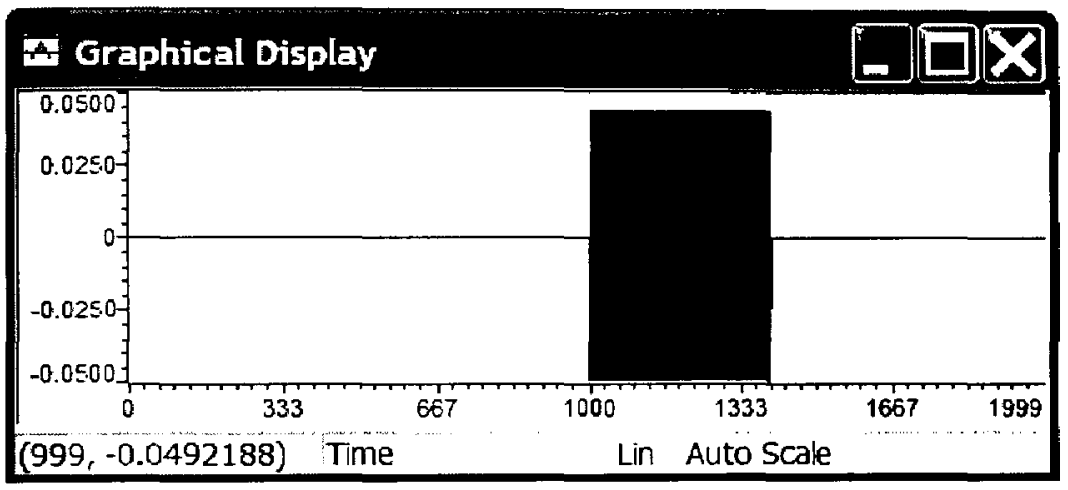

near end signal

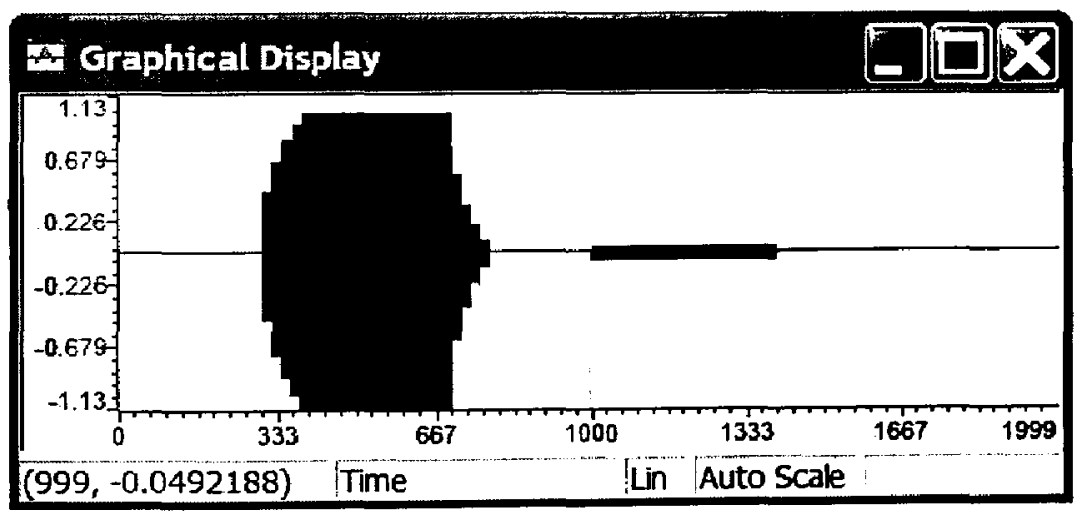

received signal 


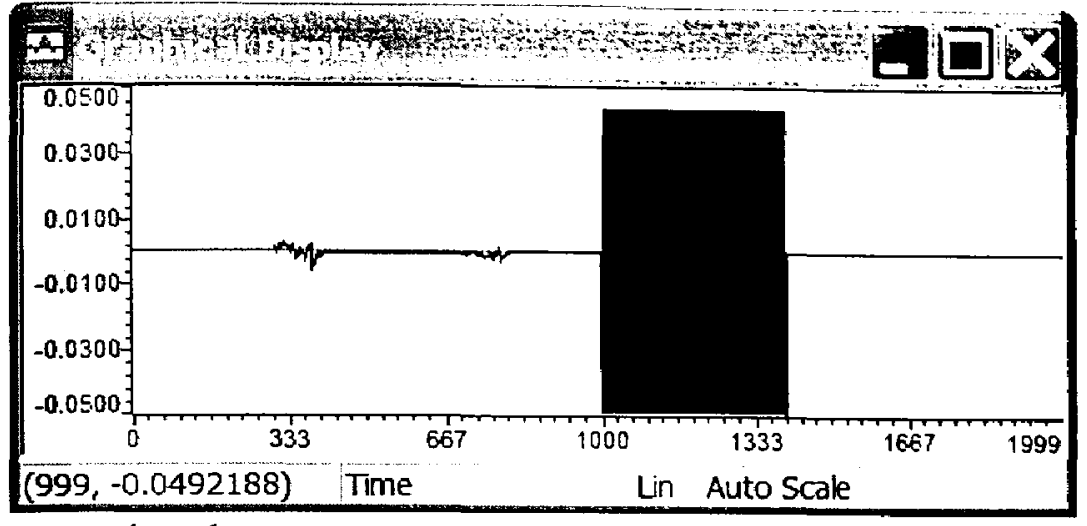

error signal

Figure 7.9 Non-convergence, in-band signaling, and continuity check tones

\begin{tabular}{|l|l|l|}
\hline Far end/near end Input & $\begin{array}{l}\text { Variation } \\
\text { Requirement }\end{array}$ & $\begin{array}{l}\text { Result } \\
\text { (NLP Enabled) }\end{array}$ \\
\hline $2400 / 2400 \mathrm{~Hz}$ & $\pm 2 \mathrm{~dB}$ & meet req. \\
\hline
\end{tabular}

Table 7.7 Non-convergence, in-band signaling, and continuity check tones test results

\subsection{Real time simulation summary}

Test 1: Steady state residual and residual echo level test

\begin{tabular}{|l|l|l|l|}
\hline Input & Output Req. & Result & Matlab Result \\
\hline$-10 \mathrm{dBm} 0$ & $-37 \mathrm{dBm} 0$ & $-44 \mathrm{dBm} 0$ & $-46 \mathrm{dBm} 0$ \\
\hline
\end{tabular}

Test 2C: Convergence test in the presence of background noise

\begin{tabular}{|l|l|l|l|l|}
\hline Input & Noise & Output Req. & Matlab Result & Result \\
\hline$-10 \mathrm{dBm} 0$ & $-30 \mathrm{dBm} 0$ & $-30 \mathrm{dBm} 0$ & $-46 \mathrm{dBm} 0$ & $-44 \mathrm{dBm} 0$ \\
\hline
\end{tabular}

Test 3: Performance under conditions of double talk

Test 3A

\begin{tabular}{|l|l|l|l|l|}
\hline Input & Near end & Output Req. & Matlab Result & Result \\
\hline$-10 \mathrm{dBm} 0$ & $-25 \mathrm{dBm} 0$ & $-25 \mathrm{dBm} 0$ & $-34 \mathrm{dBm} 0$ & $-33 \mathrm{dBm} 0$ \\
\hline
\end{tabular}

Test 3B

\begin{tabular}{|l|l|l|l|l|}
\hline Input & Near end & Output Req. & Matlab Result & Result \\
\hline$-10 \mathrm{dBm} 0$ & $-10 \mathrm{dBm} 0$ & $-27 \mathrm{dBm} 0$ & $-35 \mathrm{dBm} 0$ & $-30 \mathrm{dBm} 0$ \\
\hline
\end{tabular}


Test 6: Non-divergence on narrow-band signals

\begin{tabular}{|l|l|l|l|}
\hline Input & Output Req. & Matlab Result & Result \\
\hline$-10 \mathrm{dBm} 0$ & $-27 \mathrm{dBm} 0$ & $-32 \mathrm{dBm} 0$ & $-34 \mathrm{dBm} 0$ \\
\hline
\end{tabular}

Test 7: Stability test

\begin{tabular}{|l|l|l|l|}
\hline Input & Output Req. & Matlab Result & Result \\
\hline$-20 \mathrm{dBm} 0$ & $-47 \mathrm{dBm} 0$ & $\begin{array}{l}-48 \mathrm{dBm} 0 \\
@-10 \mathrm{dBm} 0 \text { input }\end{array}$ & $-58 \mathrm{dBm} 0$ \\
\hline
\end{tabular}

Test 8: Non-convergence, in-band signaling, and continuity check tones

\begin{tabular}{|l|l|l|}
\hline $\begin{array}{l}\text { Far end/near end Input } \\
\gamma_{2}\end{array}$ & $\begin{array}{l}\text { Variation } \\
\text { Requirement }\end{array}$ & $\begin{array}{l}\text { Result } \\
\text { (NLP Enabled) }\end{array}$ \\
\hline $2400 / 2400$ & $\pm 2 \mathrm{~dB}$ & meet req. \\
\hline
\end{tabular}

Table 7.8 Real time simulation summary 


\section{Chapter 8}

\section{Conclusion}

In this project, four adaptive algorithm LMS, NLMS, Fast Block LMS and RLS are investigated and simulated using AEC Analysis and Design program in Matlab for echo cancellation. Comparing these algorithms, NLMS is finally selected as the best algorithm for the real time echo cancellation systems. In double talk detection, the Geigel algorithm and the normalized cross-correlation DTD algorithm are separately integrated with NLMS FIR filter against double talk and are studied in NLMS \& DTD Analysis and Design program in Matlab. The normalized cross-correlation DTD algorithm is selected for double talk detection, since the results are much better. Through testing with ITU G.168 standard, the performance of the adaptive echo cancellation system conforms with the standard. In addition, the real time adaptive echo cancellation simulation by TI TMS320C6711 also proves the selected algorithms and their settings are correct. 


\section{Chapter 9}

\section{Further Work}

There are some possibilities for further development in this project, some of these are as follows.

- The double talk detection algorithm still can be done further studies in the simplified smoothing estimation algorithm area to improve the alarm accuracy and reduce the computation complexity.

- The real time echo cancellation system is successfully developed using the TI TMS320C6711 DSK. However, since this system is code in C language, if it is in assembler, the system performance will be much improved and If the TI C5000 DSP using Fixed-Point Digital Signal Processor, the same performance can be achieved with the lower cost.

- Instead of the DSK board, the adaptive echo cancellation system can use the TMS320C6711 digital signal processor in a custom designed circuit by which will also improve the whole system performance. 


\section{References}

[1] C.F.N. Cowan, P.M. Grant, Adaptive Filters, Prentice-Hall, New Jersey, 1985.

[2] Paulo S.R. Diniz, Adaptive Filtering, Algorithms and Practical implementation, Kluwer Academic Publishers, Boston, 1997.

[3] D. P. Mandic, "A Generalized Normalized Gradient Descent Algorithm," IEEE Signal Processing Letters, vol. 11, pp. 115-118, February 2004.

[4] D. L. Duttweiler, “A twelve-channel digital echo canceler," IEEE Trans. Communication, vol. 26, pp. 647-653, May 1978.

[5] J. Benesty, D. R. Morgan, and J. H. Cho, "A new class of doubletalk detectors based on cross-correlation," IEEE Trans. Speech Audio Processing, vol. 8, pp. 168172, March 2000.

[6] J. H. Cho, D. R. Morgan, and J. Benesty, "An objective technique for evaluating doubletalk detectors in acoustic echo cancelers," IEEE Trans. Speech Audio Processing, vol. 7, pp. 718-724, Nov. 1999.

[7] P. Ahgren, "A new doubletalk detection algorithm with a very low computational complexity," Submitted to IEEE Trans. Speech Audio Proc., December 2003.

[8] J. Benesty, D. R. Morgan, and J. H. Cho, "A new class of doubletalk detectors based on cross-correlation," IEEE Trans. Speech Audio Processing, March 2000.

[9] H. Ye and B.-X. Wu, "A new double-talk detection algorithm based on the orthogonality theorem," IEEE Trans. Commun., vol. 39, pp.1542-1545, Nov. 1991.

[10] T. G"ansler, M. Hansson, C.-J. Invarsson, and G. Salomonsson, "A double-talk detector based on coherence," IEEE Trans. Commun., vol.44, pp. 1421-1427, Nov. 1996.

[11] Michael L. Honig, David G. Messerschmitt, Adaptive Filters, Structures, Algorithms and Applications, Kluwer Academic Publishers, Boston, 1984.

[12]C. Antweiler and M. Dörbecker, "Perfect sequence excitation of the NLMS algorithm and its application to acoustic echo control," Annales des Telecommunications, no. 7-8, pp. 386-397, July-August 1994.

[13] Chassaing, Rulph, DSP applications using $C$ and theTMS320C6x DSK, John Wiley and Sons, New York, 2002. 
[14] P. Heitkamper, "An adaptation control for acoustic echo cancellers," IEEE Signal Processing Lett., vol. , pp. 170-172, June 1997.

[15] B. Farhang-Boroujeny, Adaptive Filters, Theory and Applications, John Wiley \& Sons, 1998.

[16] Texas Instruments: Code Composer Studio/ TMS320C6711, Documentation CD accompanying theTMS320C6711 DSP Starter Kit, 2002.

[17] Dave Bell, "How to Begin Development With the TMS320C6711 DSP," Texas Instruments Application Notes, Available: http://focus.ti.com/lit/an/spra522/spra522.pdf, March 1999. 


\section{APPENDIX A: MATLAB CODE}

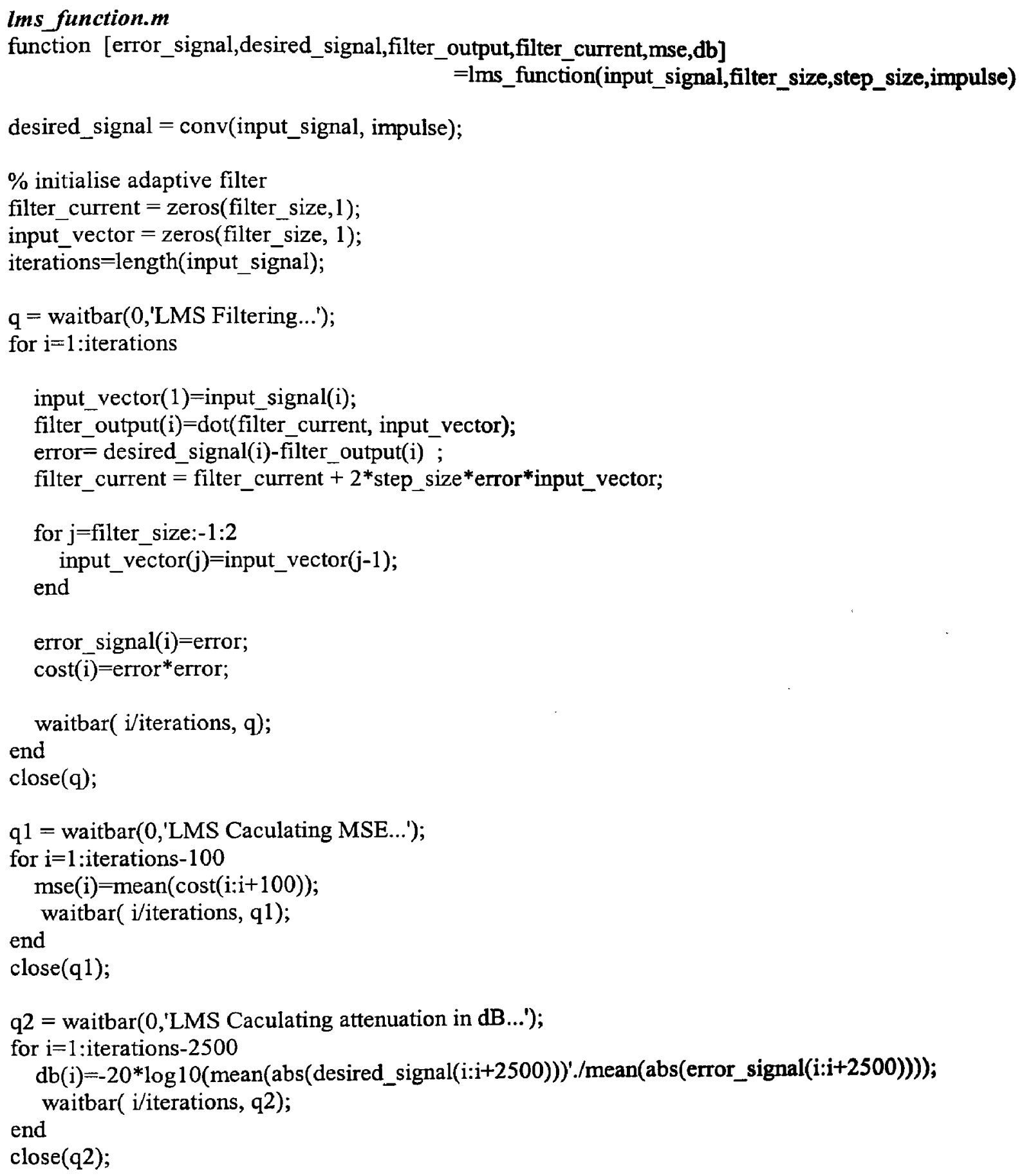




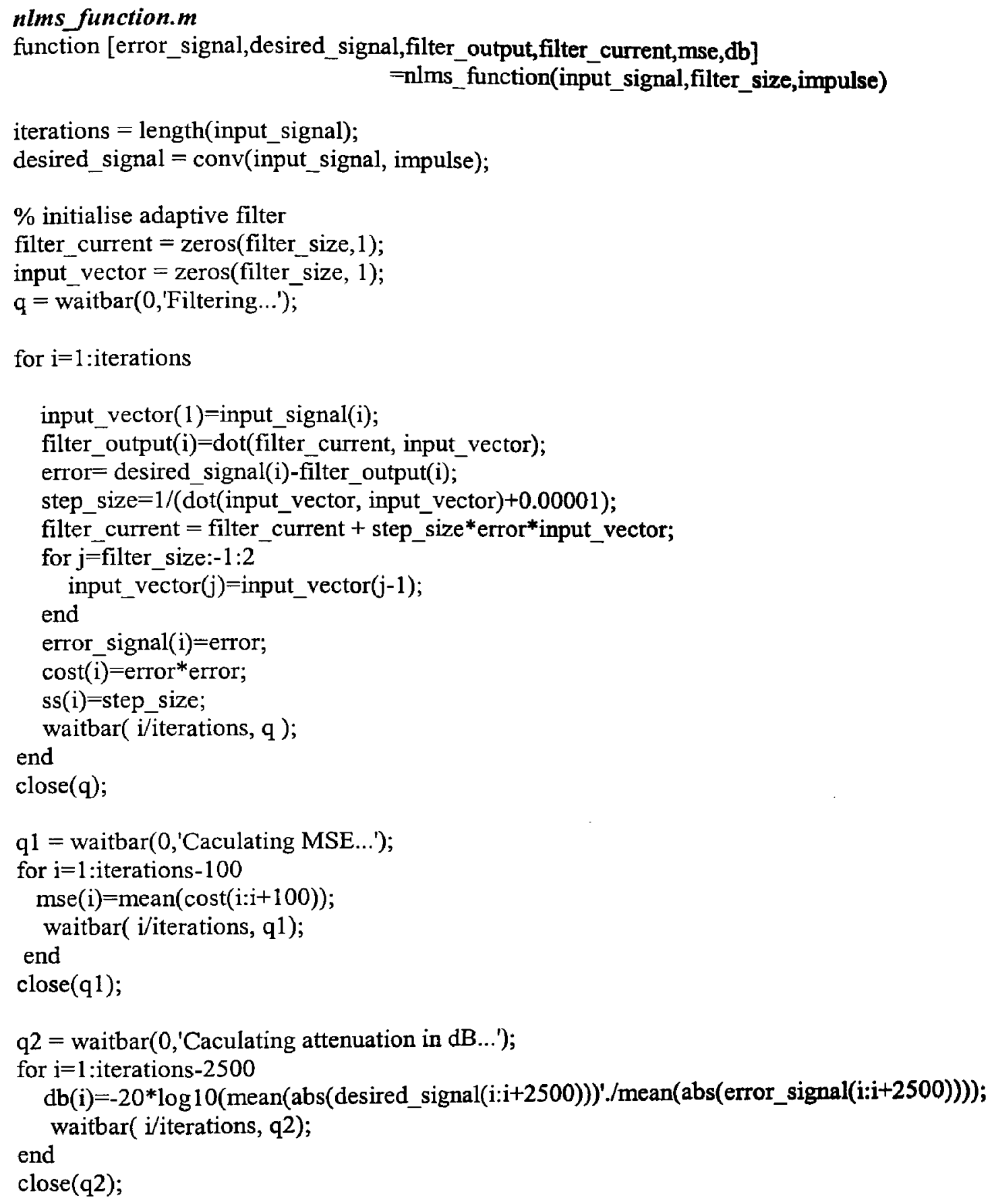




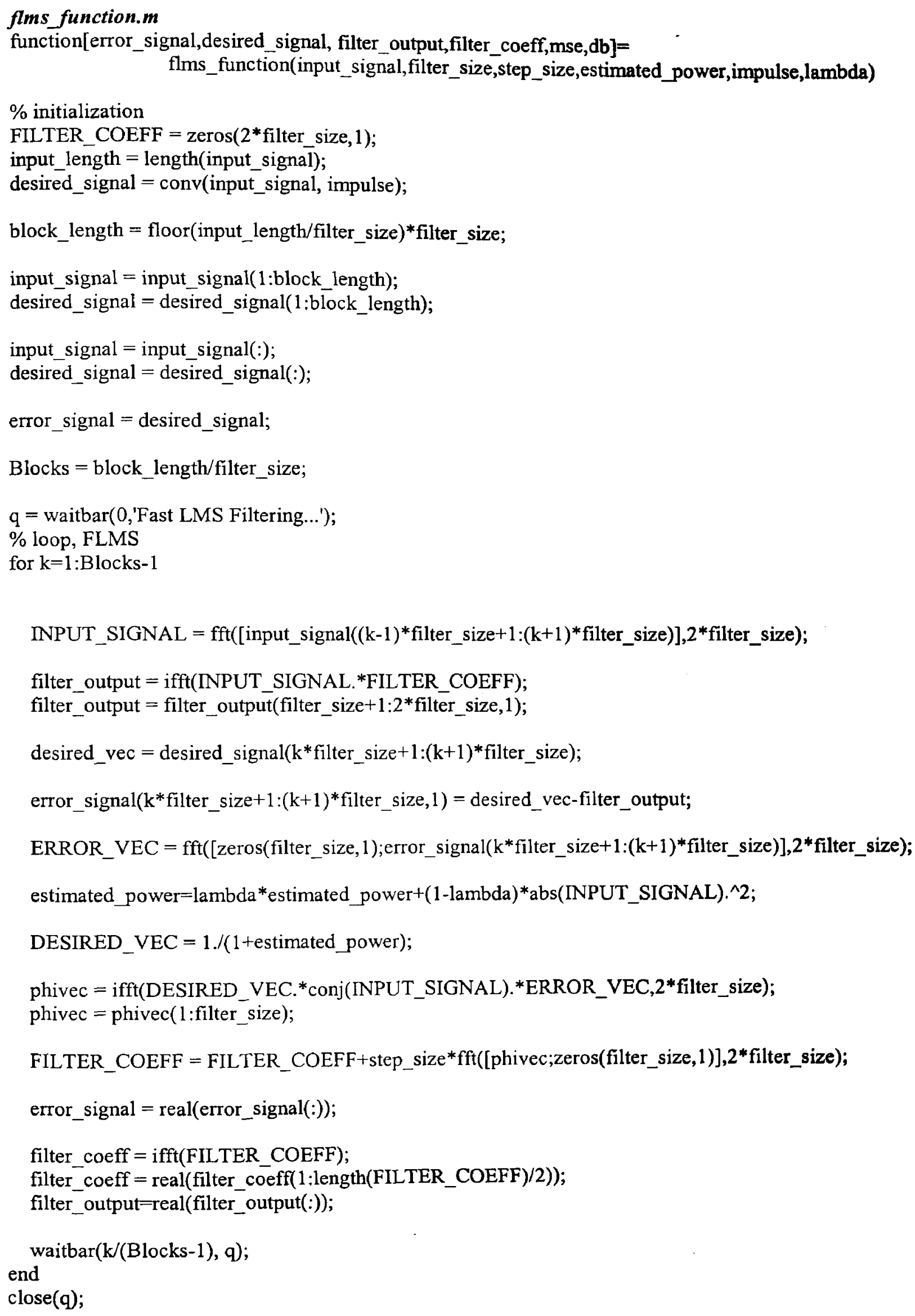


cost=error_signal.*error_signal;

q1 = waitbar (0,'Fast LMS Caculating MSE...');

iterations=length(cost);

for $\mathrm{i}=1$ : iterations -100

mse $(i)=\operatorname{mean}(\operatorname{cost}(\mathrm{i}: \mathrm{i}+100))$;

waitbar( $\mathrm{i}$ /iterations, $\mathrm{q} 1$ );

end

$\operatorname{close}(\mathrm{q} 1)$;

q2 = waitbar( 0 ,'Fast LMS Caculating attenuation in $\mathrm{dB} . .$. ');

iterations=length(desired_signal);

for $\mathrm{i}=1$ :iterations -2500

$\mathrm{db}(\mathrm{i})=-20 * \log 10($ mean(abs(desired_signal(i:i+2500)))'./mean(abs(error_signal(i:i+2500)))); waitbar( $\mathrm{i} /$ iterations, q2);

end

close(q2); 


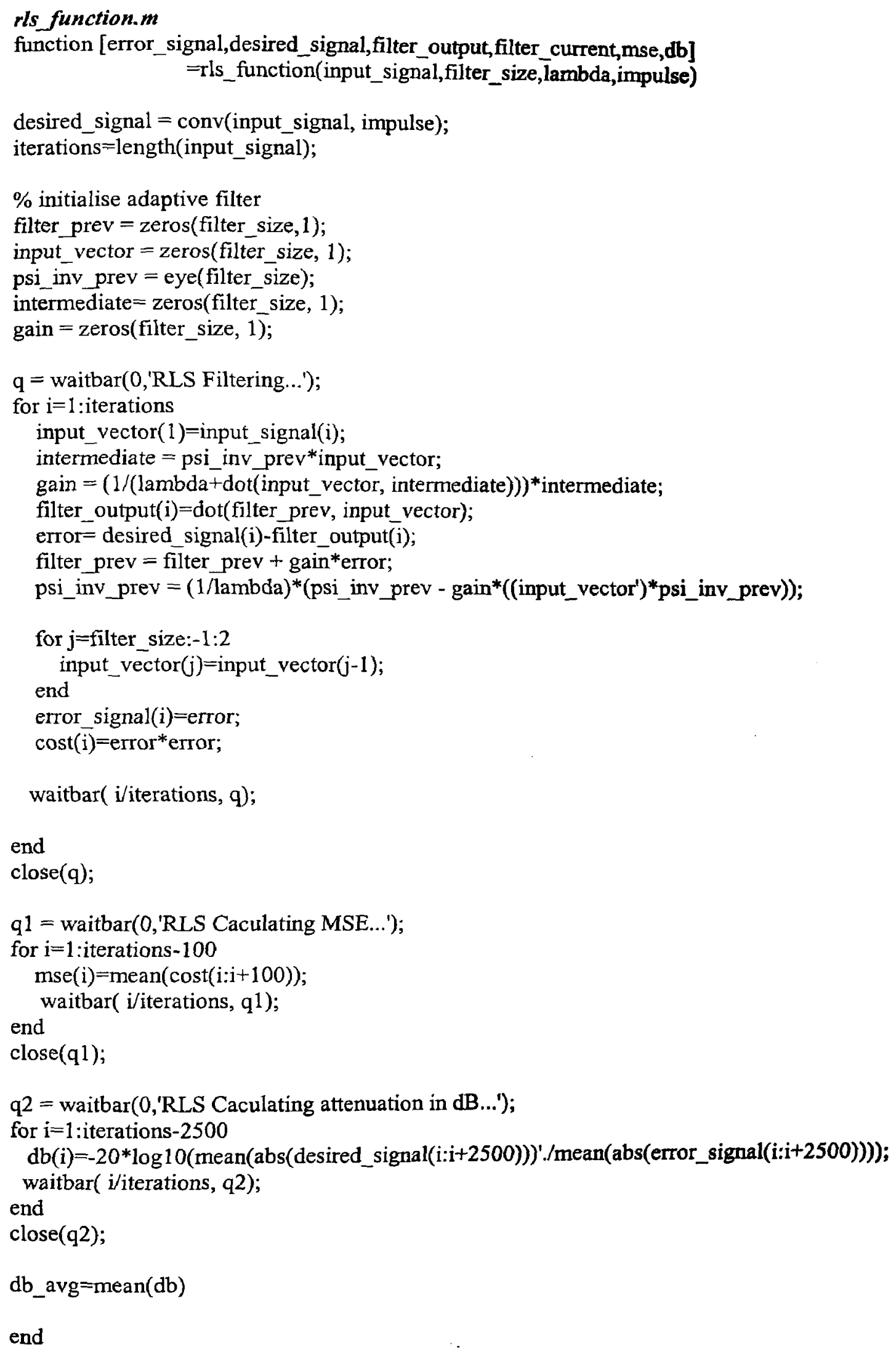




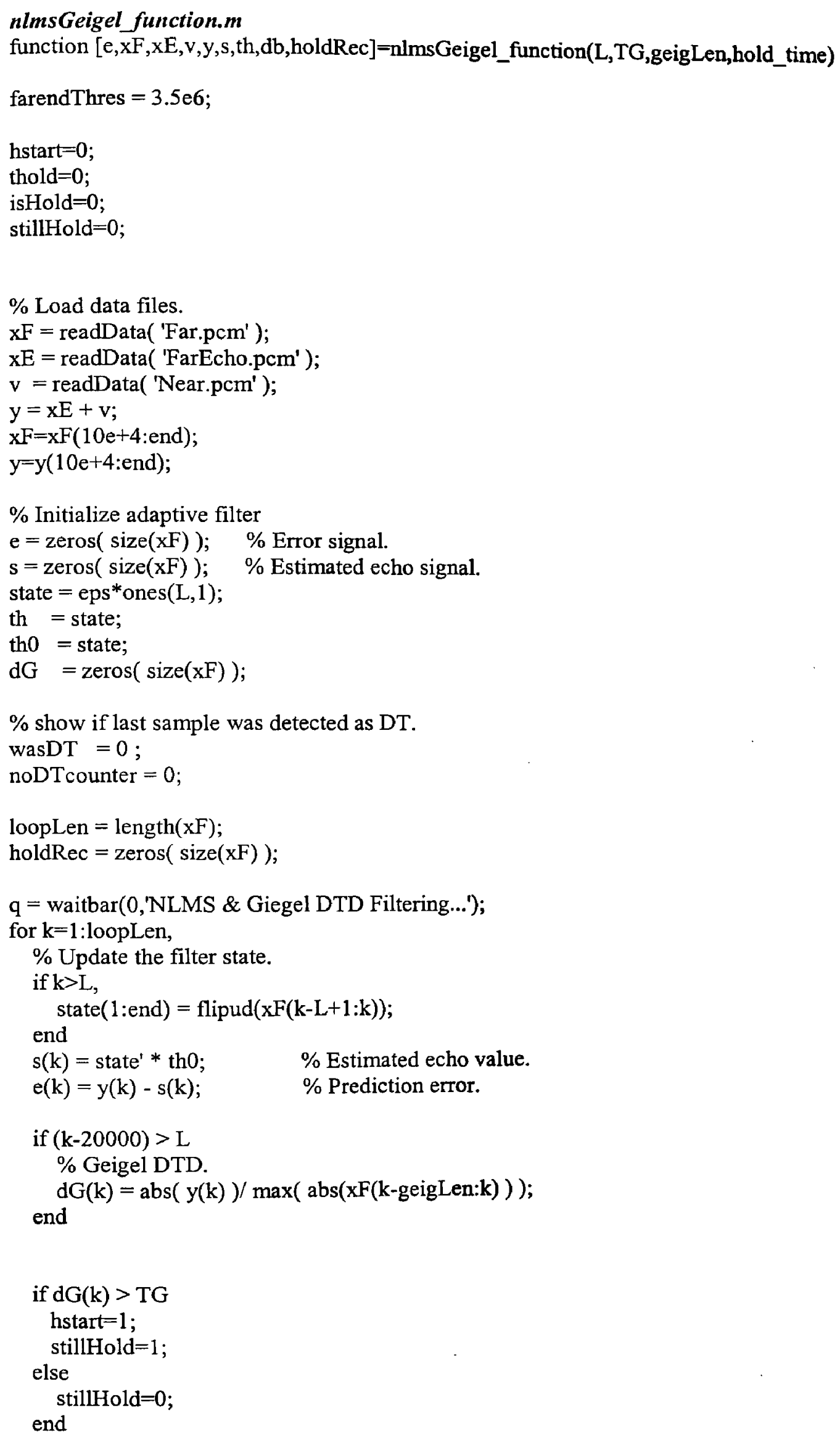




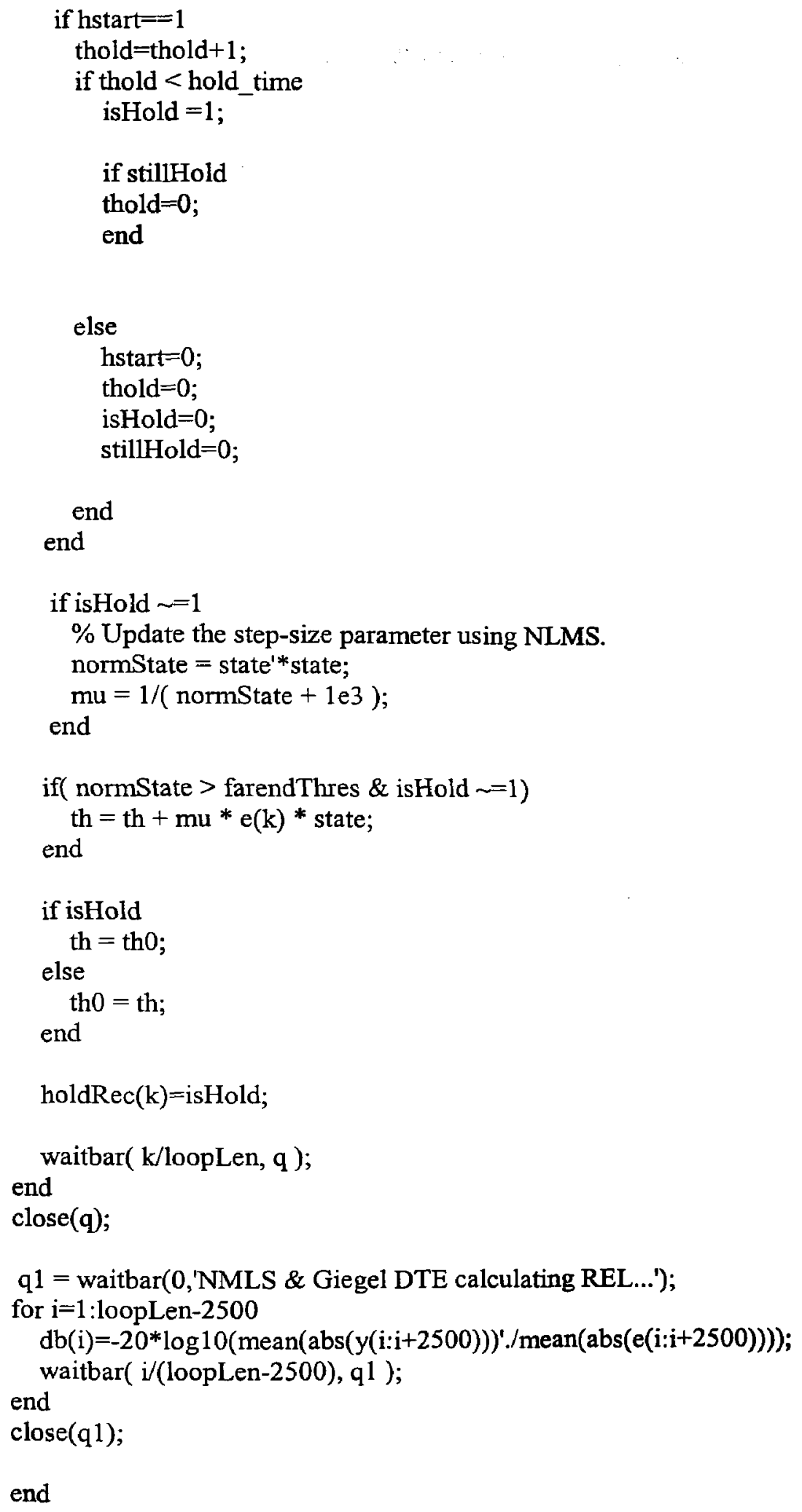




\section{nIms NCC function.m}

function [e, $\mathrm{xF}, \mathrm{xE}, \mathrm{v}, \mathrm{y}, \mathrm{s}, \mathrm{th}, \mathrm{db}$, holdRec] $=$ nlmsNCC_function(L,dt_low,dt_high,cv_hold,dt_hold)

$\mathrm{L}=1024$;

farendThres $=3.5 \mathrm{e} 6$;

$\%$ Load data files.

$\mathrm{xF}=$ readData ( 'Far.pcm' );

$\mathrm{xE}=$ readData( 'FarEcho.pcm' );

$\mathrm{v}=\operatorname{readData}($ 'Near.pcm' );

$y=x E+v$

$\%$ Initialize adaptive filter

$\mathrm{e}=\operatorname{zeros}(\operatorname{size}(\mathrm{xF})) ; \quad \%$ Error signal.

$\mathrm{s}=\operatorname{zeros}(\operatorname{size}(\mathrm{xF})) ; \quad \%$ Estimated echo signal.

state $=$ eps ${ }^{*}$ ones $(\mathrm{L}, 1)$;

th = state;

th0 = state;

$\mathrm{dG}=\operatorname{zeros}(\operatorname{size}(\mathrm{xF}))$;

$\%$ show if last sample was detected as DT.

wasDT $=0$;

noDTcounter $=0$;

loopLen $=$ length $(\mathrm{xF})$;

$\mathrm{dtm}=\operatorname{zeros}(\operatorname{size}(\mathrm{xF}))$;

$\mathrm{rxy}=\mathrm{zeros}(\mathrm{L}, 1)$;

isDT $=0$;

$\mathrm{dv}=1$;

convergeCounter $=$ zeros $(1000,1)$;

convergeFlag $=0$;

rxy $=0$;

$\mathrm{i}=0$;

startFlag $=0$;

is Coverge $=0$;

wasCoverge $=0$;

hstart $=0$;

thold $=0$;

isHold $=0$;

holdRec $=$ zeros $(\operatorname{size}(x F))$;

stillHold $=0$;

$q=$ waitbar(0,'NMLS \& NCC: Filtering...');

for $\mathrm{k}=1$ :loopLen,

$\%$ Update the filter state.

if $\mathrm{k}>\mathrm{L}$,

state $(1:$ end $)=$ flipud $(x F(k-L+1: k))$;

end

$\mathrm{s}(\mathrm{k})=\mathrm{state}^{\prime} *$ th0; $\quad$ \% Estimated echo value.

$\mathrm{e}(\mathrm{k})=\mathrm{y}(\mathrm{k})-\mathrm{s}(\mathrm{k}) ; \quad$ \% Prediction error.

if $\mathrm{k}>2 * \mathrm{~L} \& \mathrm{rxy}=0$

for $\mathbf{m}=0: L-1$, 


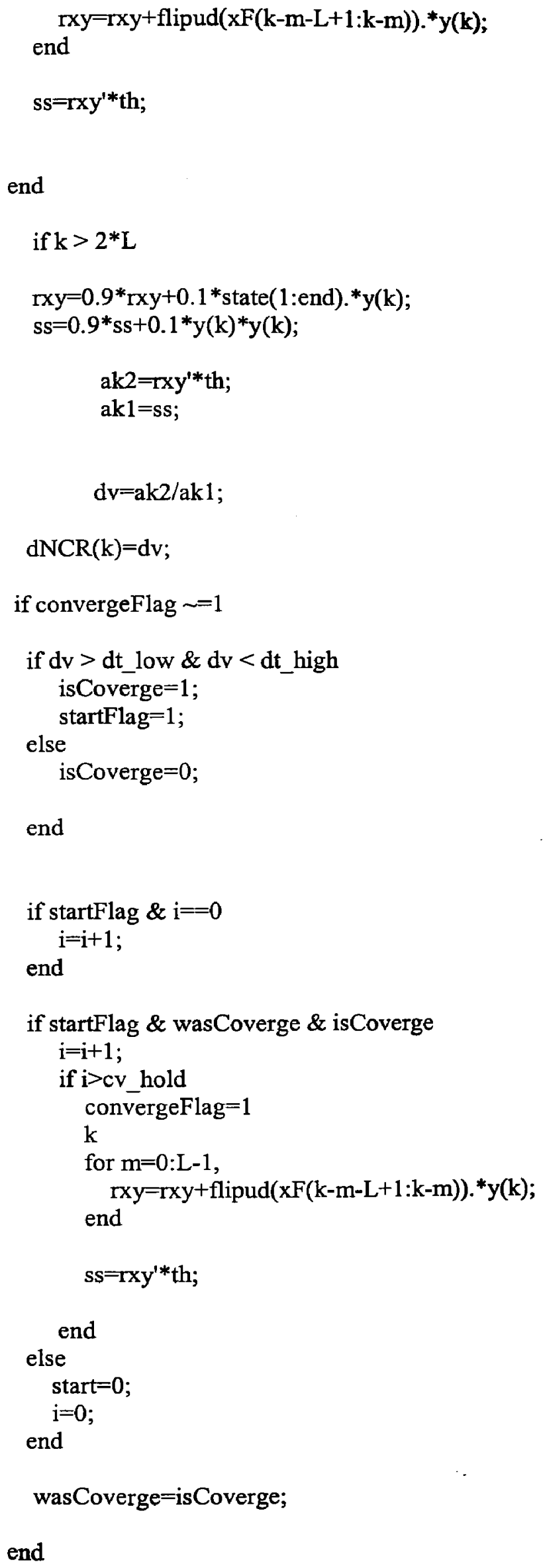




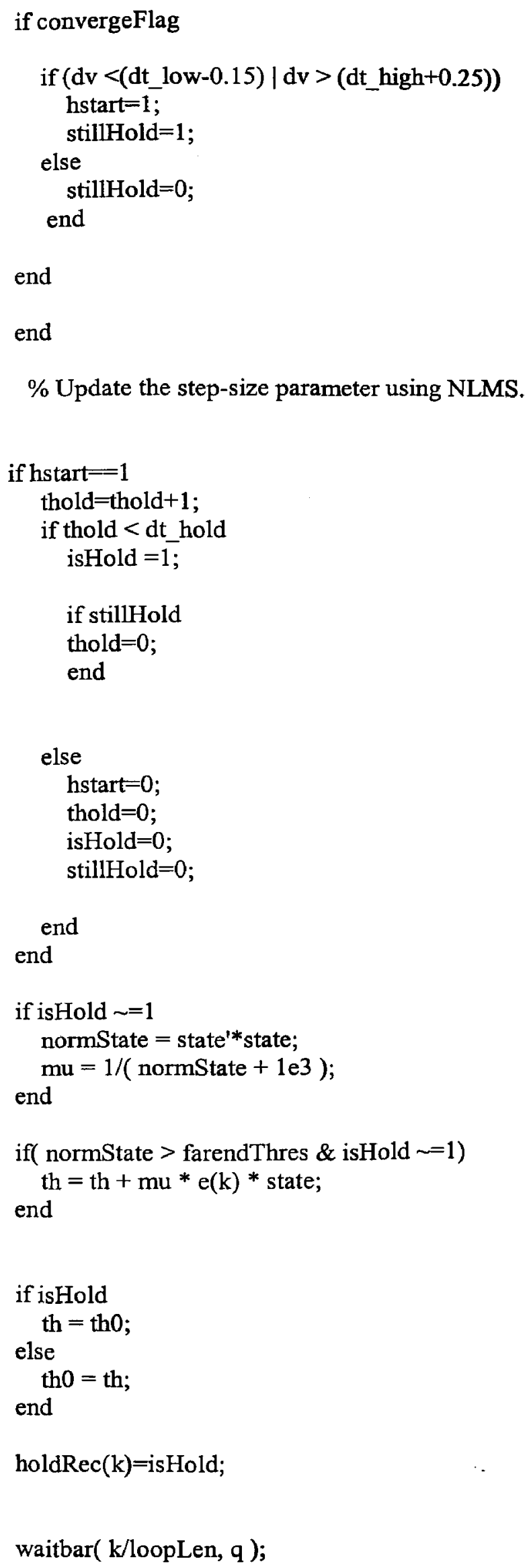


end

close(q);

q1 = waitbar(0,'NLMS \& NCC: calculating ERLE...);

for $i=1$ :loopLen-2500

$\mathrm{db}(\mathrm{i})=-20 * \log 10\left(\operatorname{mean}(\operatorname{abs}(\mathrm{y}(\mathrm{i}: \mathrm{i}+2500)))^{\prime} \cdot / \operatorname{mean}(\mathrm{abs}(\mathrm{e}(\mathrm{i}: \mathrm{i}+2500)))\right)$; waitbar( $\mathrm{i} /($ loopLen-2500), $\mathrm{q} 1$ );

end

close(q1); 


\section{APPENDIX B: TI CCS CODE}

nlms_adfilt_result.c

\#include <math.h $>$

\#include <stdio.h>

\#include <stdlib.h>

\#include "filter.h"

// Defines

\#define LOOP_LENGTH 2000

\#define FILTER_LENGTH 100

// Declare variables

float input, echoed, af_output, error_signal;

float input_signal[LOOPP_LENGTH];

float echoed_signal[LOOP_LENGTH];

float error_signal_array[LOOP_LENGTH];

//int dtd_array[LOOOP_LENGTH $]$;

float input_vector[FILTER_LENGTH];

float input_vector_c[FILTER_LENGTH];

float filter[FILTER_LENGTH];

float filter_hold[FILTER_LENGTH];

float rxy[FILTER_LENGTH];

float nstep_size;

short output;

short i, j, k,m;

//Declare variables for double talk detection

float tmp, $\mathrm{sd}=0.0, \mathrm{ak} 2=0.0, \mathrm{dv}=0.0$;

int hstart $=0$, stillHold $=0$, isHold $=0$, thold $=0$, is NLP $=0$;

// Procedure for determining dot product of two arrays

float dotp (float $\mathrm{a}[]$, float $\mathrm{b}[]$ )

\{

float suml, sumh;

suml $=0$;

sumh $=0$;

for $\left(j=0 ; j<F I L T E R \_L E N G T H ; j+=2\right)$

\{

$\operatorname{suml}+=a[j] * b[j]$

\}

sumh $+=a[j+1] * b[j+1]$;

\}

return (suml+sumh);

//Procedure for determining dot product of one array and one value void dotpv(float $\mathrm{a}[]$, float $\mathrm{b}$ ) 


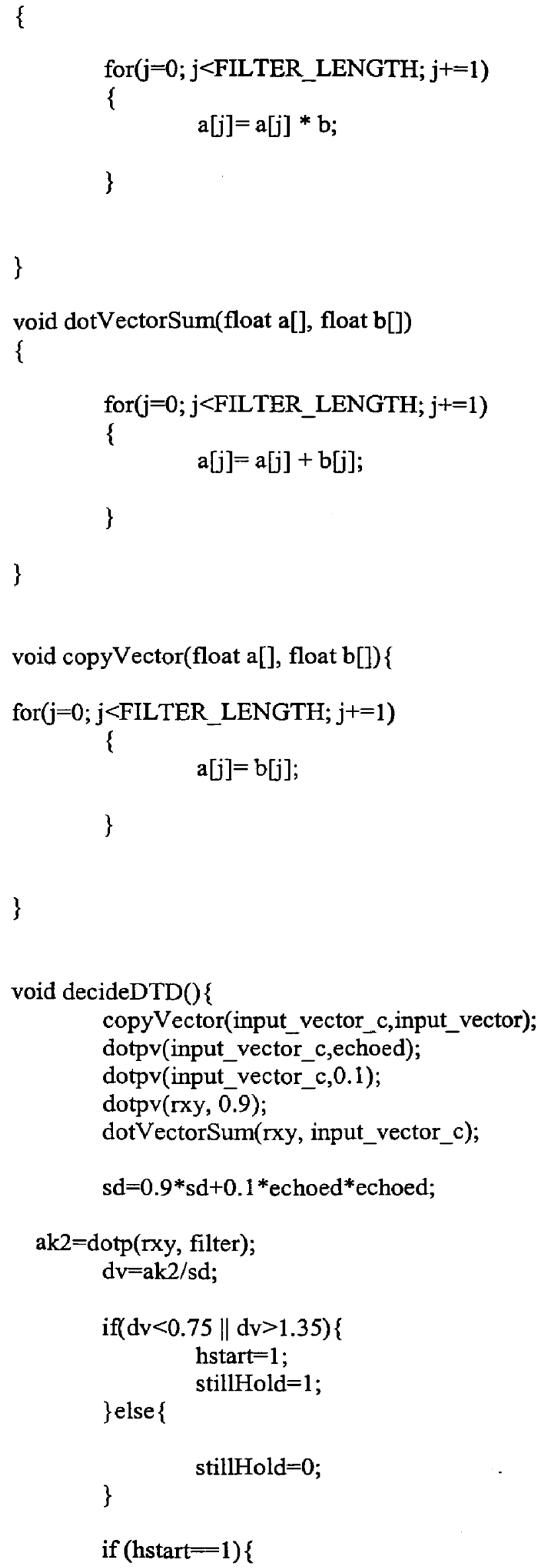




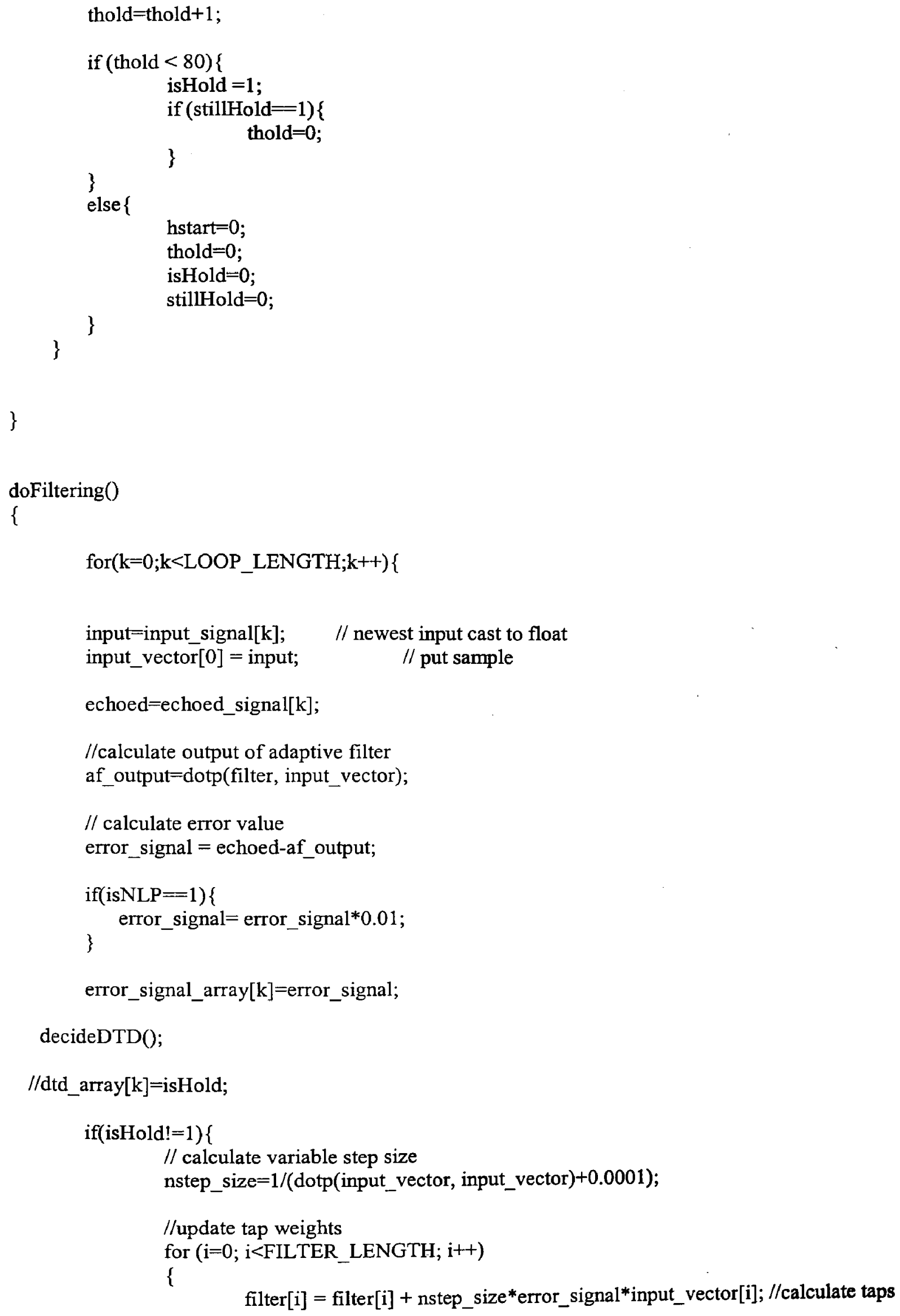




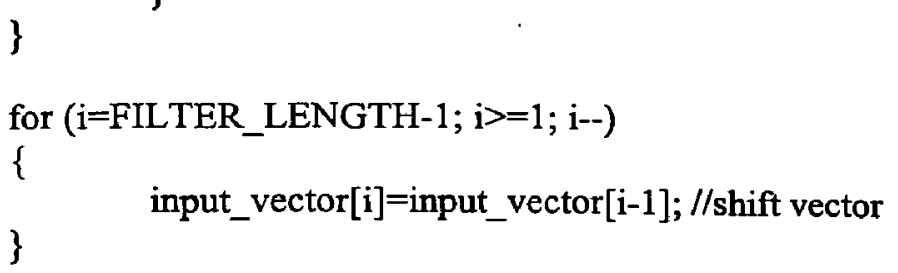

\}//for loop end

// This is main procedure executed on start up of program main()

\{

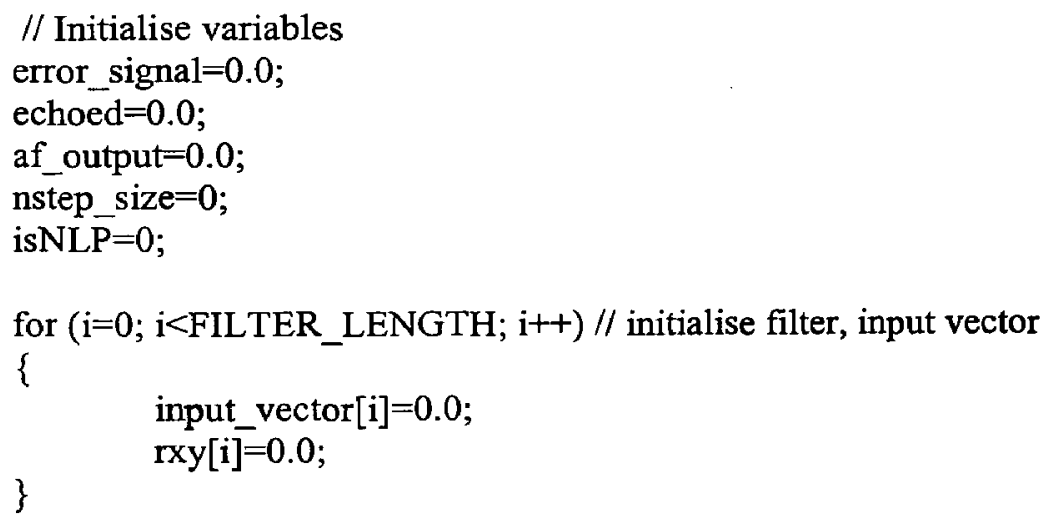

